\title{
WestVirginiaUniversity
}

THE RESEARCH REPOSITORY @ WVU

Graduate Theses, Dissertations, and Problem Reports

2005

\section{Aircraft intercom system design for project oculus}

Jay Paul Wilhelm

West Virginia University

Follow this and additional works at: https://researchrepository.wvu.edu/etd

\section{Recommended Citation}

Wilhelm, Jay Paul, "Aircraft intercom system design for project oculus" (2005). Graduate Theses,

Dissertations, and Problem Reports. 4204.

https://researchrepository.wvu.edu/etd/4204

This Thesis is protected by copyright and/or related rights. It has been brought to you by the The Research Repository @ WVU with permission from the rights-holder(s). You are free to use this Thesis in any way that is permitted by the copyright and related rights legislation that applies to your use. For other uses you must obtain permission from the rights-holder(s) directly, unless additional rights are indicated by a Creative Commons license in the record and/ or on the work itself. This Thesis has been accepted for inclusion in WVU Graduate Theses, Dissertations, and Problem Reports collection by an authorized administrator of The Research Repository @ WVU. For more information, please contact researchrepository@mail.wvu.edu. 
Aircraft Intercom System Design for Project Oculus

By

Jay Paul Wilhelm

Thesis submitted to the College of Engineering and Mineral Resources

at West Virginia University

in partial fulfillment of the requirements

for the degree of

Master of Science

in

Electrical Engineering

Approved by

Dr. Roy S. Nutter, Jr., Committee Chairperson

Dr. James E. Smith

Dr. Powsiri Klinkhachorn

Lane Department of Computer Science and Electrical Engineering

Morgantown, West Virginia

2005

Keywords: C-130, Aircraft Intercom, Project Oculus 


\section{Abstract \\ Aircraft Intercom System Design for Project Oculus}

\section{Jay Paul Wilhelm}

Project Oculus is an ongoing development project at WVU that aims to create a quick and easy sensor platform for deployment on the C-130 aircraft. Because this platform will be operated inside the noisy cargo bay of the C-130, an intercom system needed to be designed in the operator station of Project Oculus that would allow for different types of headsets and future expandability.

An intercom system was designed, constructed, and tested that uses military and civilian headsets to communicate both internally for Project Oculus and externally to the C-130 crew and provides support for a headset to be connected externally of the operator station. 


\section{Acknowledgements}

I would like to thank WVU Center for Industrial Applications (CIRA) for funding this project and this thesis. Most of all I would like to thank Dr. Roy Nutter and Dr. James Smith for allowing me to work on an exciting and ever developing project. Also Franz (Andy) Pertl, Emily Pertl, Zenovy Wowczuk, Gerald (Bear) Angle, and Dr. Klinkhachorn for keeping on my case and making sure things got done. 


\section{Table of Contents}

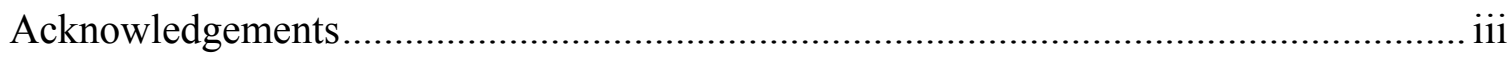

Table of Contents ............................................................................................. iv

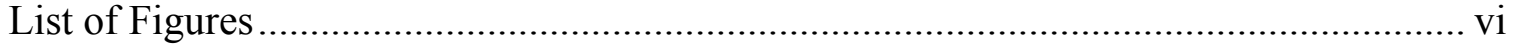

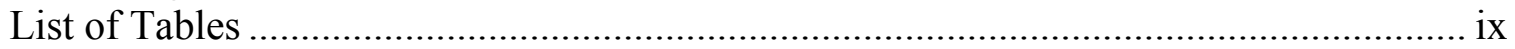

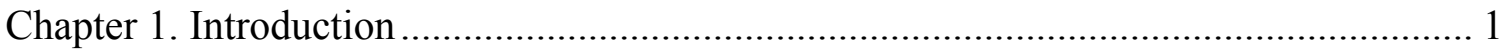

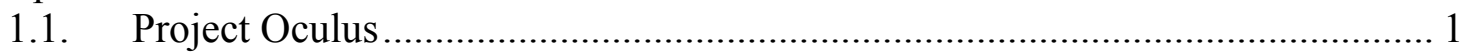

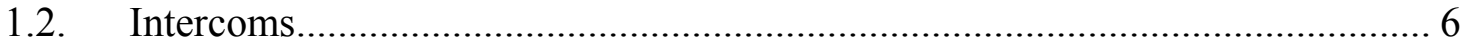

Chapter 2. Statement of the Problem ........................................................................ 8

Chapter 3. Aircraft Intercoms ……………………….......................................... 9

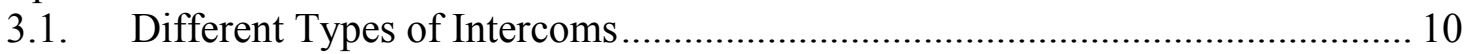

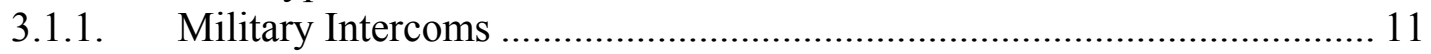

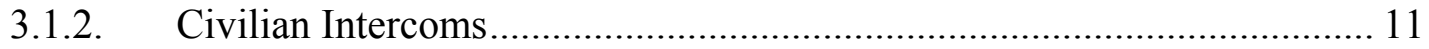

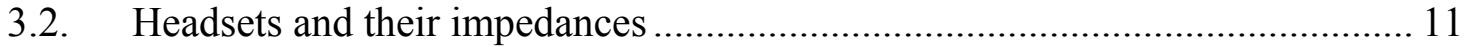

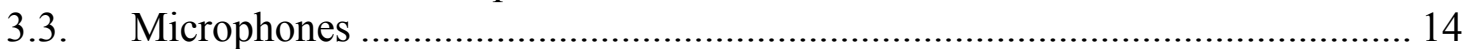

Chapter 4. Intercom Design for Project Oculus.......................................................... 17

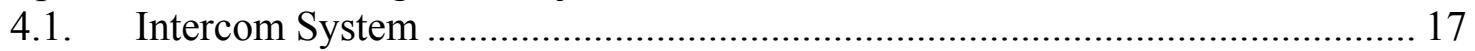

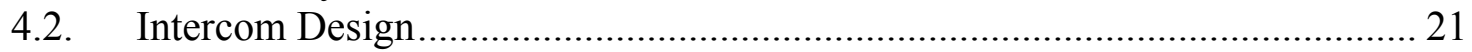

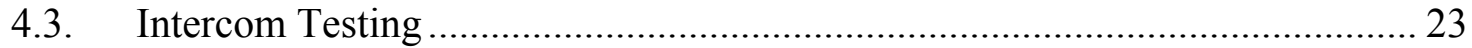

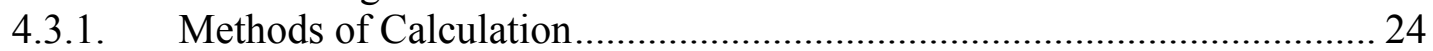

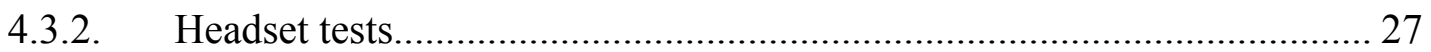

4.3.3. Impedance Matching Circuitry Tests ........................................................ 35

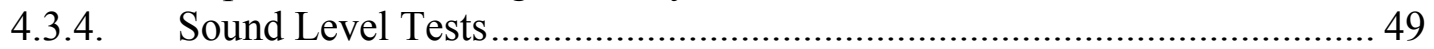

4.3.5. Dynamic Microphone Amplifier Circuit Tests .......................................... 51

4.3.6. Overall Tests Conclusion.................................................................... 56

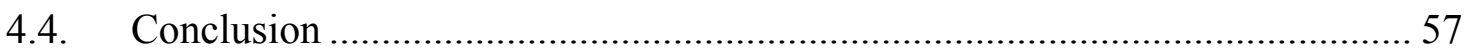

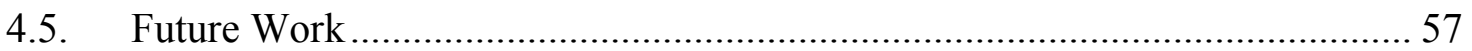

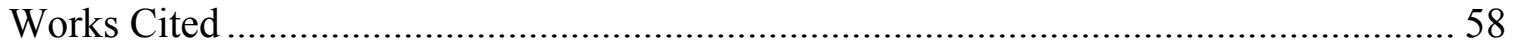

Appendix A Custom Impedance Matching Circuit ......................................................... 60

A.1 Headset Impedance Matching Circuit Design .................................................... 60

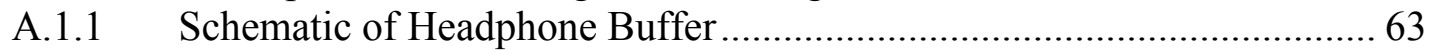

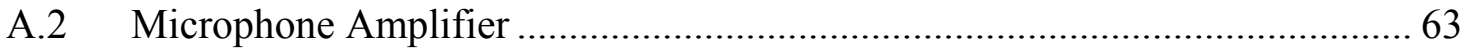

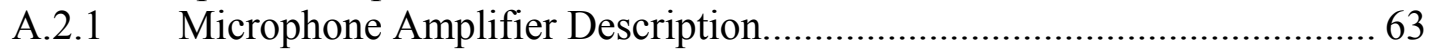

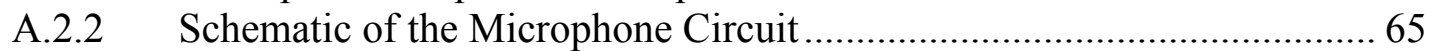

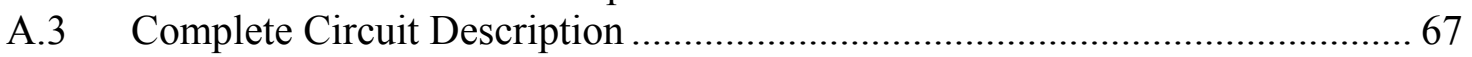

A.4 Schematic and Layout of Impedance Matching Circuit .................................... 73

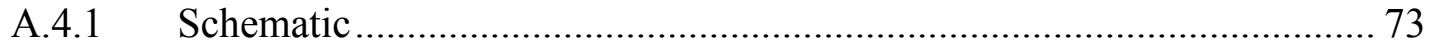

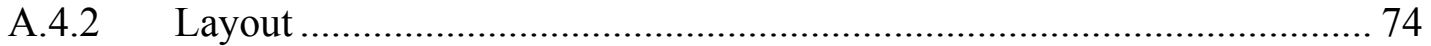

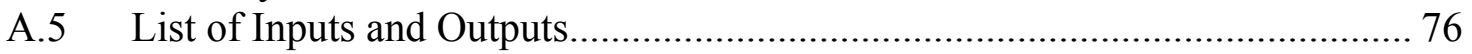

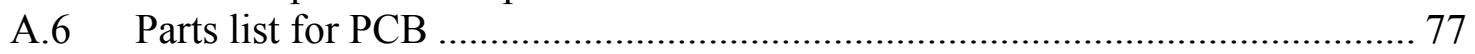

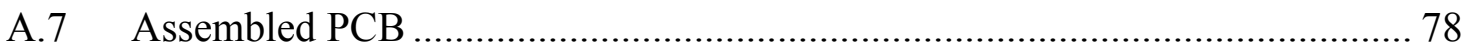

A.8 Various Measured Frequency Responses and other Tests ................................ 78

Appendix B Project Oculus Intercom Schematics........................................................... 79 


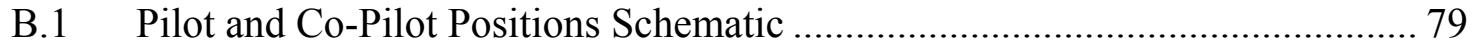

B.2 Middle Position Schematic ...................................................................... 80

B.3 Intercom Tie Line Connections ................................................................. 81

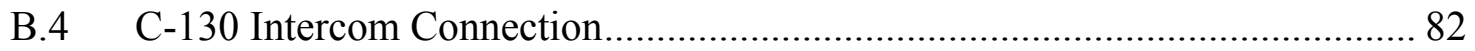

B.5 Panel Layout of Project Oculus Intercom System ........................................ 83

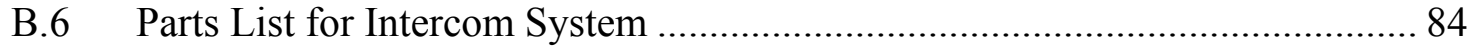

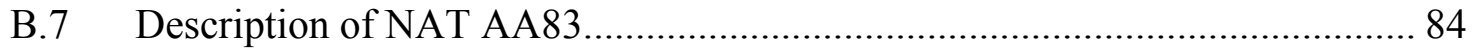

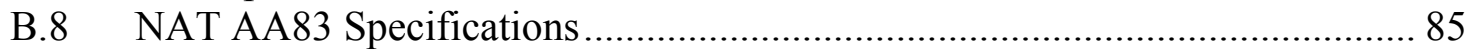

B.9 Description of NAT AA36-100 …............................................................ 86

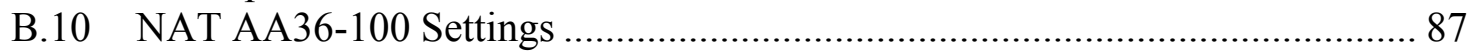

B.11 Flightcom Denali ANR \& Civilian Headset Specifications [18]................... 88

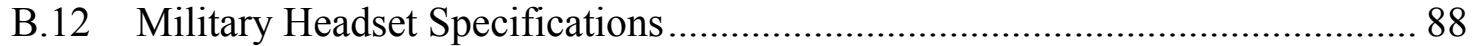

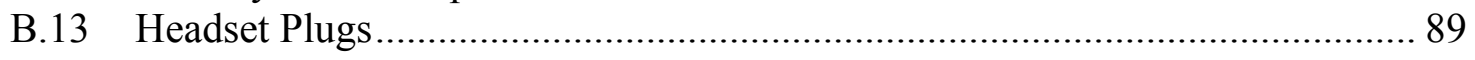




\section{List of Figures}

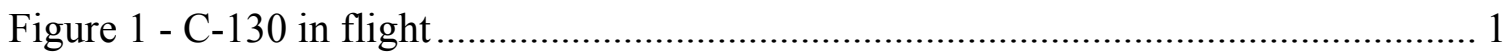

Figure 2 - Sensor Pallet with sensor pod extended [4] .................................................... 2

Figure 3 - Operator Station Pallet [2] ......................................................................... 3

Figure 4 - Standardized sensor pallet system concept diagram [2] …………………..... 4

Figure 5 - Pendant Control................................................................................... 5

Figure 6 - Various Headset Speaker configurations ………………………………..... 13

Figure 7 - Dynamic Microphone............................................................................. 14

Figure 8 - Electret Condenser Microphone...………………………………………... 15

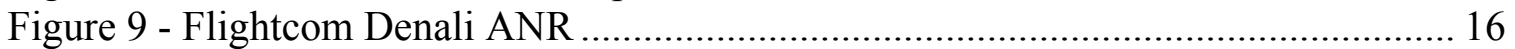

Figure 10 - Flightcom Denali ANR Military ............................................................... 16

Figure 11 - Concept Headset Selection Device per Operator Station................................. 19

Figure 12 - Basic Block Diagram of Intercom Connections.............................................. 22

Figure 13 - Intercom Testing Tree …………………………………………..... 23

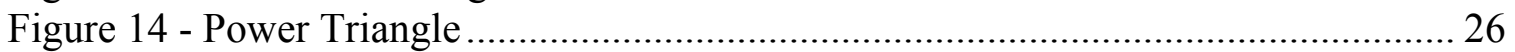

Figure 15 - AA83 Intercom Test Setup using Microphone Input ................................... 28

Figure 16 - Voltage vs Frequency AA83 Microphone Input............................................ 29

Figure 17 - Frequency Response of AA83 using $150 \& 300 \Omega$ Speaker Loads ............... 29

Figure 18 - Calculated Voltage Gain of AA83 with $150 \& 300 \Omega$ Speaker Loads .......... 30

Figure 19 - Calculated Apparent Power@125mVrms to $150 \& 300 \Omega$ Speaker Loads.30

Figure 20 - Calculated Apparent Power @ 500mVrms using $150 \& 300 \Omega$ Speaker Loads

Figure 21 - AA83 Intercom test Setup using Music Input............................................... 32

Figure 22 - Voltage vs Frequency to AA83 Music Input ................................................ 32

Figure 23 - Frequency Response of AA83 using 150 \& $300 \Omega$ Speaker Loads ................ 33

Figure 24 - Calculated Voltage Gain of AA83 using $150 \& 300 \Omega$ Speaker Loads......... 33

Figure 25 - Calculated Apparent Power @ 900 mVrms using $150 \& 300 \Omega$ Speaker

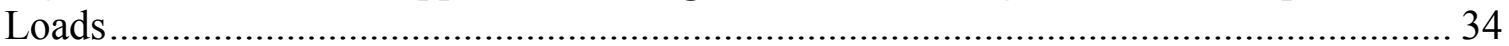

Figure 26 - Calculated Apparent Power @ 1.5 Vrms using $150 \& 300 \Omega$ Speaker Loads34

Figure 27 - Impedance box testing setup with AA83 mic input ........................................ 36

Figure 28 - Voltage input to AA83 (mic i/p) with box .................................................. 36

Figure 29 - Frequency Response of AA83 (mic i/p) with box ....................................... 37

Figure 30 - Frequency Response of box with AA83(mic i/p) ......................................... 37

Figure 31 - Calculated Voltage gain of AA83(mic i/p), box, \& overall gain ................... 38

Figure 32 - Calculated Apparent Power to $150 \Omega$ HS from box \& AA83(mic i/p) @

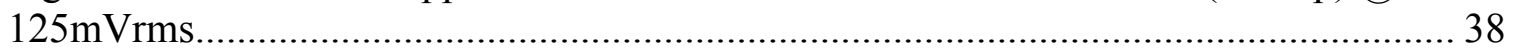

Figure 33 - Calculated Apparent Power to $150 \Omega$ HS from box \& AA83(mic i/p) @

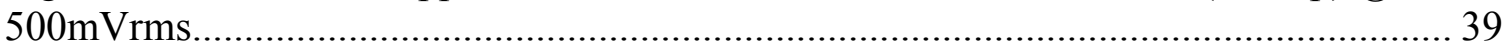

Figure 34 - Impedance box testing setup with AA83 music input ................................... 39

Figure 35 - Voltage input to AA83 (music i/p) with box .............................................. 40

Figure 36 - Frequency Response of AA83 (music i/p) with box..................................... 40

Figure 37 - Frequency Response of box with AA83(music i/p) ...................................... 41

Figure 38 - Calculated Voltage gain of AA83(music i/p), box, \& overall gain ............... 41 
Figure 39 - Calculated Apparent Power to $150 \Omega$ HS from box \& AA83 Music I/P ..... 42

Figure 40 - Calculated Apparent Power to $150 \Omega$ HS from box \& AA83 Music I/P ...... 42

Figure 41 - Impedance Box Individual Testing Setup .................................................... 43

Figure 42 - Voltage input from the Signal Generator to the Box ................................... 43

Figure 43 - Frequency Response of the impedance box ............................................ 44

Figure 44 - Frequency Response of the impedance box ............................................. 44

Figure 45 - Calculated Apparent Power to the $150 \Omega$ HS from the box......................... 45

Figure 46 - No Power impedance box testing setup ................................................... 46

Figure 47 - Input to AA83(mic i/p) \& Box without power............................................ 46

Figure 48 - Frequency Response of AA83(mic i/p) \& Box without power..................... 47

Figure 49 - Calculated Voltage Gain of AA83(mic i/p) \& Box without power ............... 47

Figure 50 - Calculated Apparent power to $150 \Omega$ HS from AA83(mic i/p) \& Box W/O

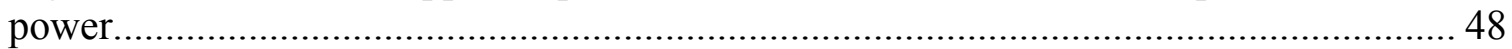

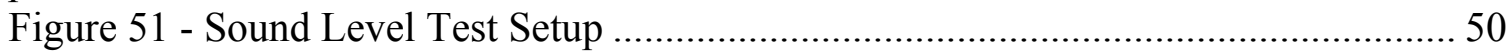

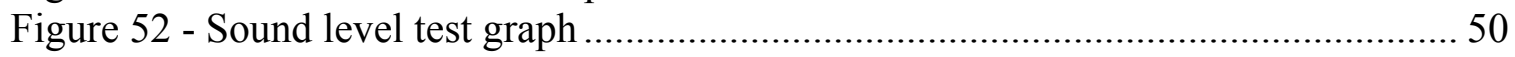

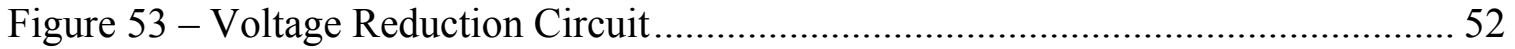

Figure 54 - Microphone Circuit Input Response Setup ............................................ 52

Figure 55 - Microphone Test Signal Generator Output ............................................. 53

Figure 56 - Microphone Test Reducer Output ............................................................. 53

Figure 57 - Microphone Test Impedance Box Output .................................................. 54

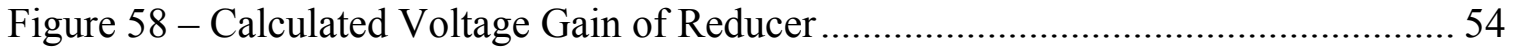

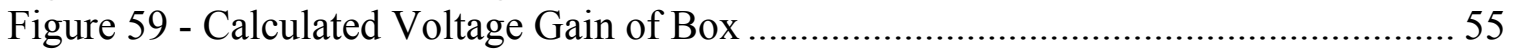

Figure 60 - Calcualted Overall Voltage Gain of Reducer \& Box ................................... 55

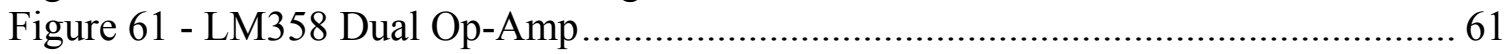

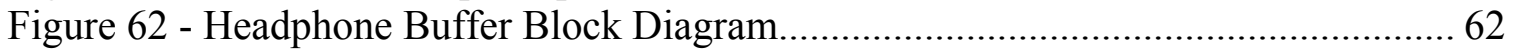

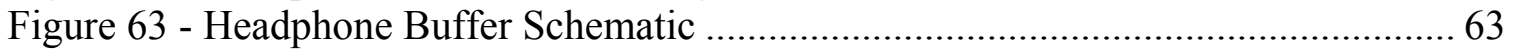

Figure 64 - LM386 Low Voltage Power Amplifier......................................................... 64

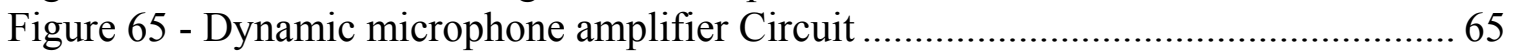

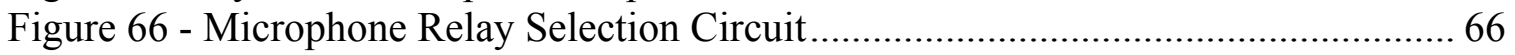

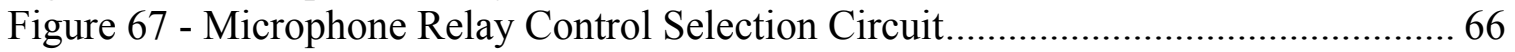

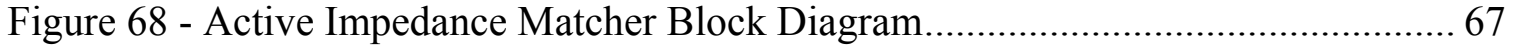

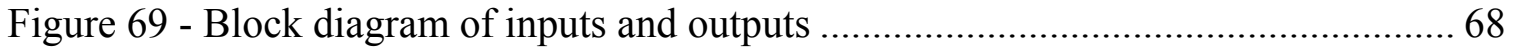

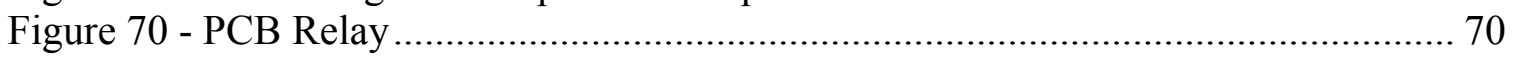

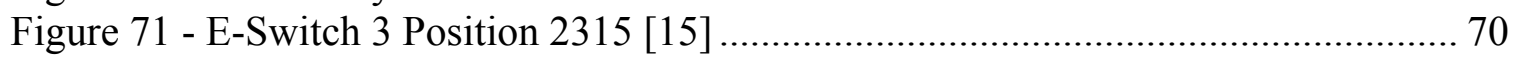

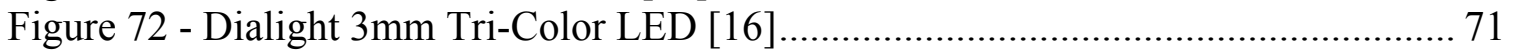

Figure 73 - Multi Connector Phoenix Contact Block [14] ......................................... 72

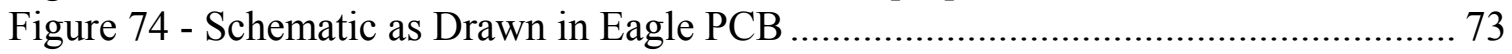

Figure 75 - Bottom Layer of PCB............................................................................ 74

Figure 76 - Top Layer of PCB ............................................................................... 74

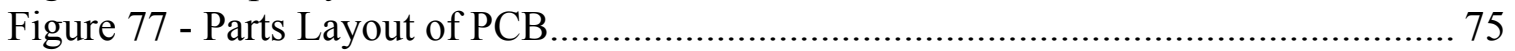

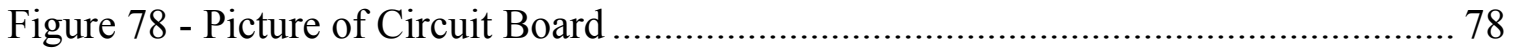

Figure 79 - Pilot/Co-Pilot Position Intercom Connection Diagram .............................. 79

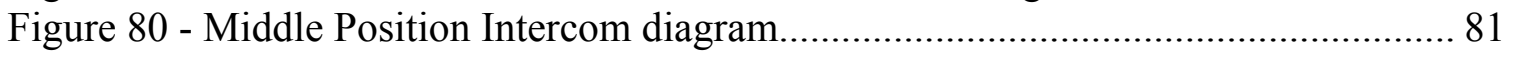

Figure 81 - Intercom Tie Line Connections............................................................ 81

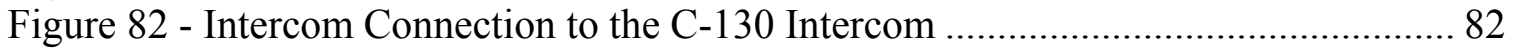

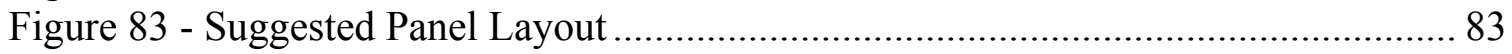




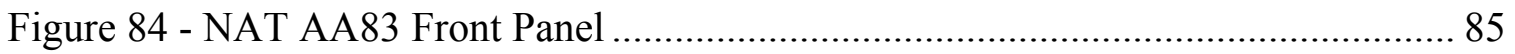

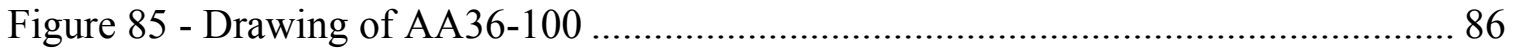

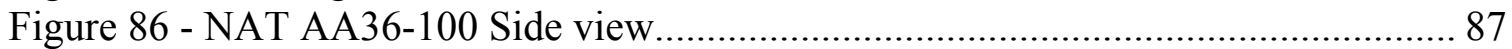

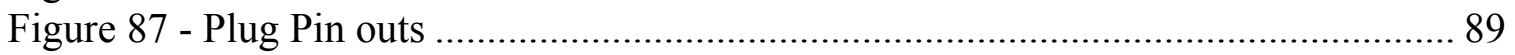

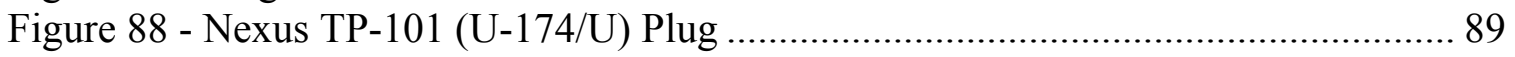

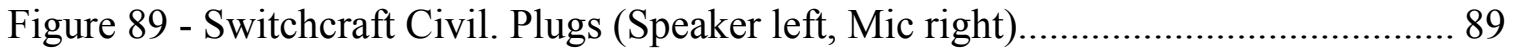

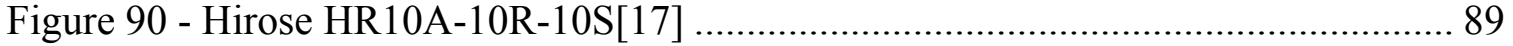

Figure 91 - Hirose HR10A-10R-10S Pinout................................................................ 89 


\section{List of Tables}

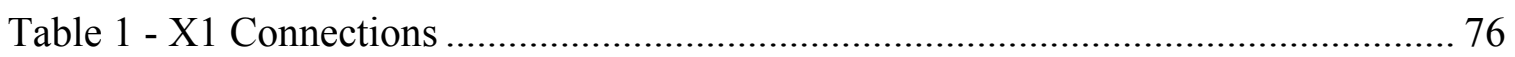

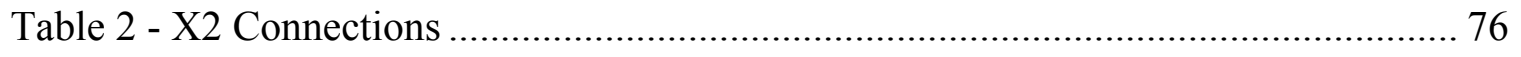

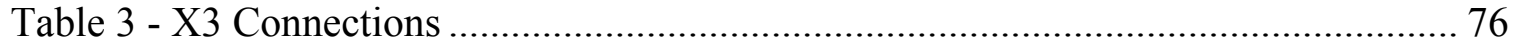

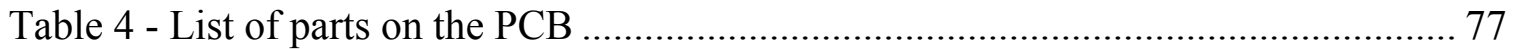

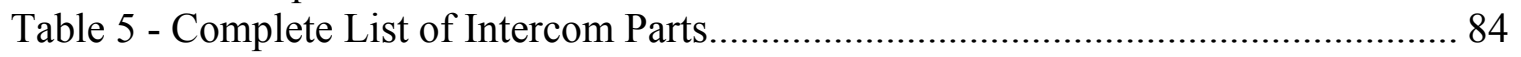

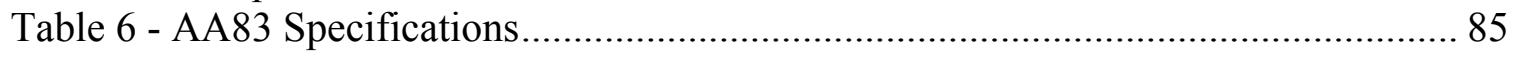

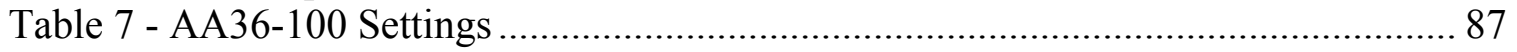




\section{Chapter 1. Introduction}

\subsection{Project Oculus}

Project Oculus is an ongoing research and development program with the goals of placing reconnaissance sensors and monitoring equipment in the cargo hold of a C-130 (Figure 1) aircraft for quick and easy deployment. Lockheed-Martin Corporation, the builder of the C-130, has produced more than 2,200 C-130s, which have been flown by more than sixty nations [1]. This makes the C-130 aircraft one of the most common cargo planes flown throughout the world and a perfect mobile platform for sensor data collection and processing.

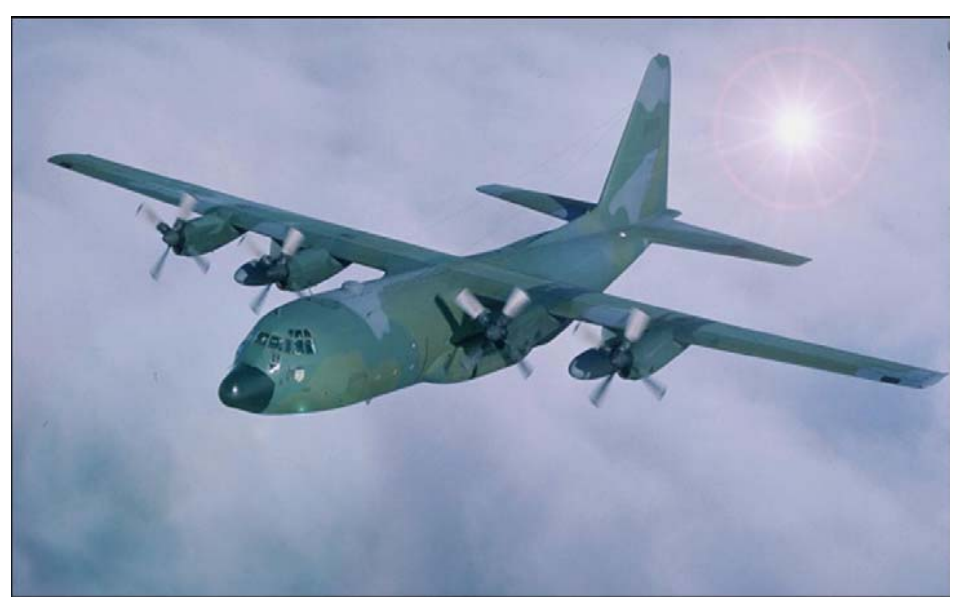

Figure 1 - C-130 in flight 
In 2003, engineers from West Virginia University’s Center for Industrial Research Applications (WVU-CIRA) proposed a design for an in-flight deployable sensor platform for use in the C-130 aircraft (Figure 2). Major design considerations were ease of installation, quick installation, ease of deployment of the pod, and construction using off the shelf parts. The main focus is a pod which locks underneath the airframe of the C-130 cargo door in flight. This pod can contain various types of sensors focusing on the ground for reconnaissance. [2]

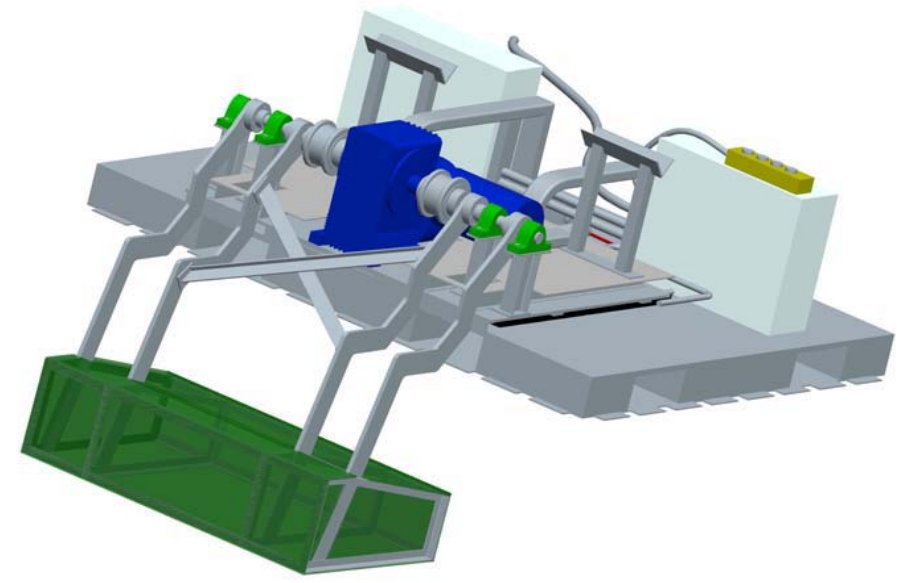

Figure 2 - Sensor Pallet with sensor pod extended [4] 
A single pallet sensor was a good design, but to make this project have more variety than from previous designs, a second pallet was designed to house sensor collection equipment, data processing equipment, and power control equipment. The second pallet is known as the operator station (Figure $3 \&$ Figure 4 ) and is placed near the center of gravity of the C-130 cargo hold. Inside the operator station is power control equipment that feeds from the 200A of 28VDC and three phase 40A of 208/110 VAC $400 \mathrm{~Hz}$ aircraft auxiliary supply lines. This provides power for both the sensor pallet and equipment in the operator station.

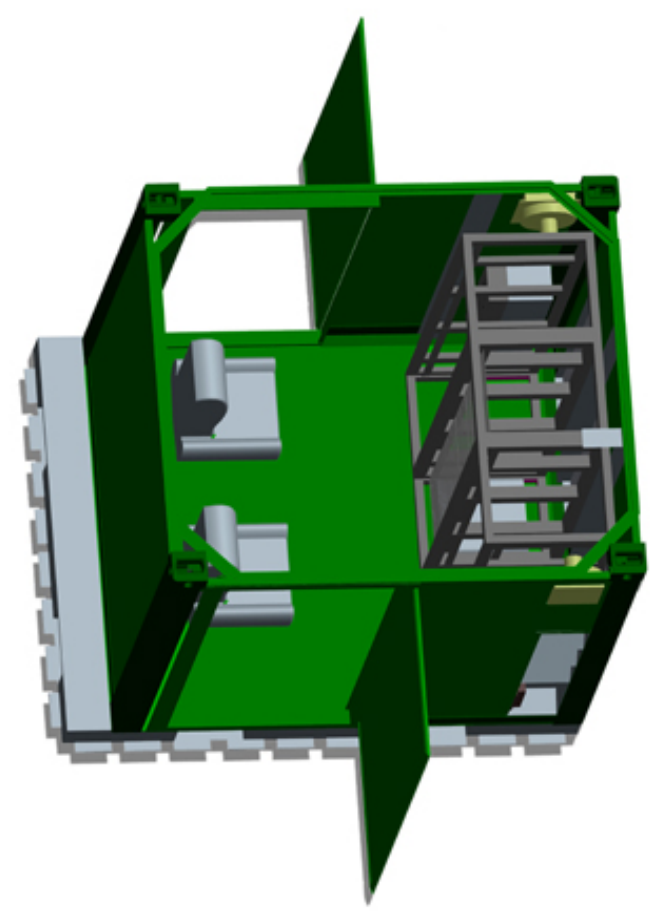

Figure 3 - Operator Station Pallet [2] 


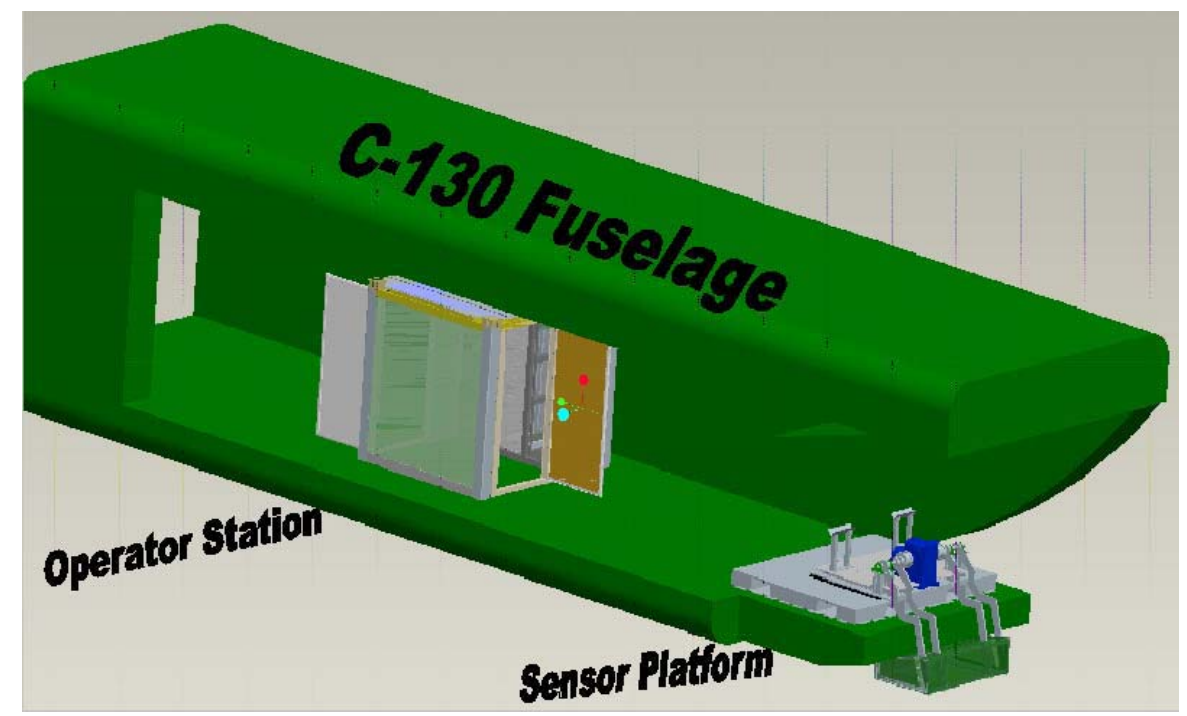

Figure 4 - Standardized sensor pallet system concept diagram [2]

The sensor pallet is normally in a stowed position, for storage and transportation, where it remains until the $\mathrm{C}-130$ is in-flight over its targeted area for data collection. The rear door of the $\mathrm{C}-130$ then opens and the translation plate begins to move out. Once the translation plate has reached its most rearward position it stops moving and the rotation of the pod starts. When the pod has rotated all the way around and down and is level with the ground, the translation plate begins moving forward. The pod will then come in contact with the underside of the door and be securely wedged into position. This deployment procedure can occur either by manual control or automatic control, which is controlled by a pendent.

The control pendent (Figure 5) consists of an emergency stop button, a switch for automatic or manual mode, a switch for rotation or translation mode, a push button for extend, and a push button for stow. The most important button on the pendent is the emergency stop button. This button, when pressed, stops all electric motors. This is a safety feature that is necessary because people or objects can get in the way of the moving parts, which may cause injury and/or malfunction. 


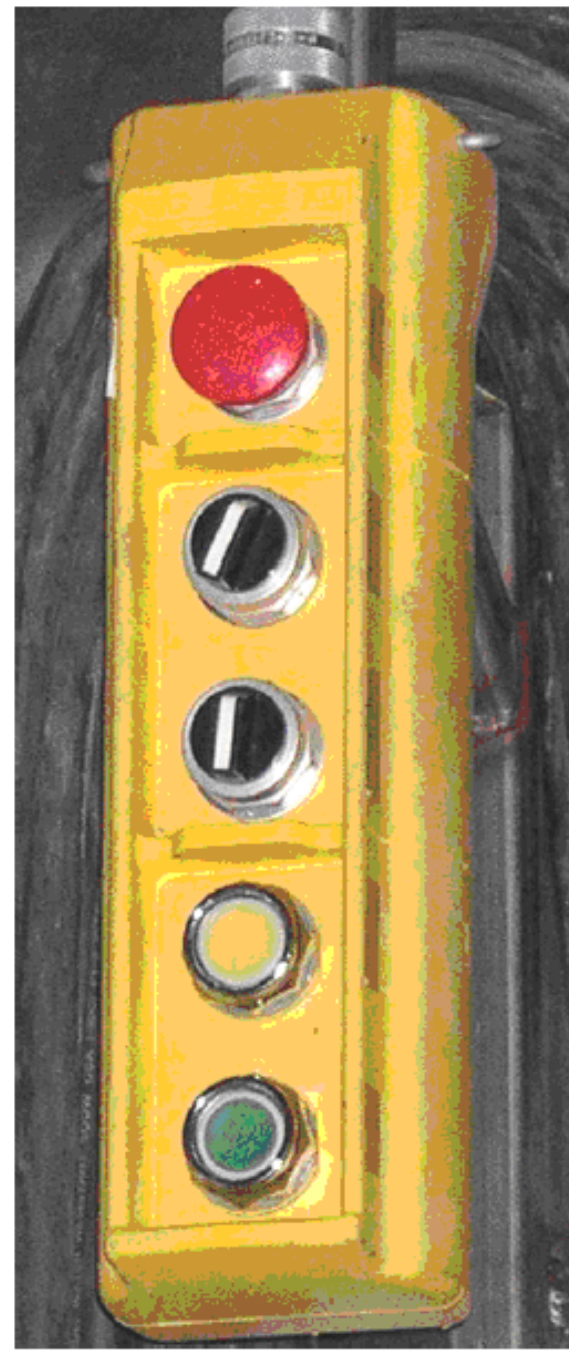

\title{
Emergency Stop Button
}

\author{
Automatic/Manual Switch
}

Translate/Rotate Switch

Extend Push Button

Stow Push Button

Figure 5 - Pendant Control

During manual control, all of the movements of the platform are controlled by selecting translation or rotation and then pressing either extend or stow buttons.

Automatic mode consists of choosing deployment or storage modes, then waiting while a PLC (Programmable Logic Controller) moves the translation plate and rotates the pod. Minimal human intervention is required to start or stop the process and the deployment and storage operations shutdown once they have been completed. Having a variety of methods allows the system to be deployed by persons who do not have discrete knowledge of exactly where each component should be located. [6] 
The concept of expandability was a major design consideration from the start of Project Oculus. Inside the operator station are three standard 19' racks that stretch from floor to ceiling allowing for equipment to be mounted. The sensor pallet also has four boxes that may contain 19" standard racks for equipment that may directly control the sensors. Inside the pod of the sensor pallet is a rack system that equipment can be connected to. All of the previously mentioned racks allow for quick and easy exchange, upgrade, or removal of equipment.

Inside the pod on the sensor pallet is where the data collection sensors are located. Each sensor can have view of the ground and can be mounted on a vibration isolation plate. The sensors range from cameras, infra-red cameras, hyper spectral cameras, antenna systems, or radar systems. The wide support and easy access to sensors provides a valuable platform for the customers that will be using the system in the field

The intention of the Project Oculus development team was to design and build two complete prototype setups, then hand the designs off for commercial construction of the pallets. This consideration made it necessary that all components be COTS (Common Off The Self) or GOTS (Government Off The Shelf) unless necessary.

\subsection{Intercoms}

Inside almost every aircraft there is an intercom system that allows the crew to communicate with each other over the loud noise of the aircraft engines and the noise generated from air flowing across the body of the craft. Inside the operators station on Project Oculus people need to communicate with each other inside the operator station, reconnaissance related radios, the $\mathrm{C}-130$ flight crew itself, sensors that can generate audio signals, and with various other audio sources. High speed computers in the operator station, that mainly process sensor data may also be used for watching movies, playing 
video games, or listening to music during the time when the collection of sensor data is not necessary such as world wide transport time to the scene of operations. These activities are common among flight crews that have nothing to do while in transit to a destination.

Adding to the complication of intercoms is the use of several different types of headsets. Headset speakers can have different impedances and headset microphones can be of completely different types with different impedances as well. This often leads to incompatibilities often resulting in low volume levels. Most aviators and sensor operators carry their own headset, which could be of any type, civilian or military, or they may sometimes carry multiple types of headsets. Aviators usually prefer to use their own personal headsets instead of ones provided by the aircraft. The most common reasons for this are sanitation and comfort.

What is needed for Project Oculus is a design of an intercom system that can allow the project operators to communicate with one another over the noise of the aircraft, communicate with the $\mathrm{C}-130$ intercom system, communicate with radios, be expandable to allow for future upgrades, and allow the operators to use whichever headsets they prefer. It was decided early because of different headset specifications that it would be necessary for most types of headsets to be used in the intercom system. 


\section{Chapter 2. Statement of the Problem}

A device does not exist off the shelf that meets the requirements of allowing both commercial and military headsets to be used on an aircraft intercom system. Project Oculus thus needed a method of allowing multiple headsets to be used with an intercom design that allows for three positions in the operator station to communicate with one another, with an external connection, and with a connection to the C-130 intercom system.

It is proposed that an intercom system be developed, constructed, and tested that can utilize military, civilian, and high quality active noise reduction headsets on the three working positions of the operator station. In addition, this intercom system must interface properly with the C-130 on-board internal communication intercom and with radios that may be installed in the operator station for exclusive use by the system operators. The load-master must in addition have a connection outside of the operator station that can communicate directly with the operators independent of the C-130 on-board intercom system when necessary. 


\section{Chapter 3. Aircraft Intercoms}

Inside almost every aircraft is an intercom system. The system might be as simple as a two person system which is also connected to a radio, or extremely complex with many headsets being supported. In previous years, headsets were connected in series to a single amplifier which had all the microphones as inputs. The system only worked well when the correct numbers of headsets were connected, and with the constraint only one person could talk at a time. Today, thanks to small components, near ideal low noise amplifiers and digital circuits, intercom systems are much easier to construct and expand.

The market today provides a designer with a plethora of different intercom systems to choose from. Although each manufacturer has unique and appealing attributes, each also has downsides. The major downside to the majority of intercoms for this project is that they are not expandable and accept only one type of headset. These types of intercom systems are ideal for the larger numbers of small aircraft that need only the minimum number of users. Fortunately for this project, Northern Airborne Technology provides intercoms that can be connected together with their Tie Line technology, which can expand the intercom system. 


\subsection{Different Types of Intercoms}

Intercom systems in a aircraft are different depending upon who makes the aircraft, when it was made, and what sector it will server. Manufacturers such as Boeing, Lockheed-Martin, Mcdonnell-Douglas, Gulfstream, Cessna, and others all have different intercom equipment for different purposes. The cargo planes the military flies, like the C130, do not have very much noise insulation, which makes the interior background noise deafening. Headsets that cover the ear must be able to overcome that noise. Commerical aircraft such as the Gulfstream G500 has noise insulation and is very quiet on the interior; therefore an intercom system is mainly used as an interface to radios and the cabin loudspeaker.

Specifications and special features of intercom systems vary depending upon the application and buyer of the aircraft. The specifications include what type of headset is meant for the intercom, how many headsets can be connected to the intercom, and what connections to radios or music sources are required.

Typically when an intercom user wishes to transmit from their microphone they must first press a "push to talk" button. The alternative to this is using VOX (voice Activation) which is the most useful and sometimes the most annoying feature. This allows a user to activate their microphone by speaking. A level of activation is usually set by a knob, so when the microphone level goes over the activation threshold, the intercom starts transmitting from the microphone. This allows a headset to be hands free, i.e. no pushing a button to talk. The main problem is that loud background noise can start the transmission, the level is set too high and the user has problems starting the transmission, or a time delay to activation is so long that the first syllabus or word of whatever the user says is cutoff. 


\subsubsection{Military Intercoms}

The military usually outfits cargo planes with simple intercom systems that lack voice activation and music inputs. The majority of these intercom systems were designed in the 1960 's when vacuum tubes were the only solution, and the constructed intercoms still exist on aircraft today.

The C-130 aircraft has a military specification intercom control box type AN/AIC-18 [7]. This box accepts military headsets of $150 \mathrm{ohm}$ speaker impedance and is monorail. The microphone input to this box is required to be a dynamic microphone. Inside the cockpit of the C-130 are military headset connections for the pilot, co-pilot, navigator, and others. These headsets all communicate together or each connection can communicate with a radio. Also there are several connections in the cargo hold of the C130 that can communicate with the cockpit. Included also in the cargo hold are numerous other intercom connections that allow many headsets to communicate with other headsets that are in the cargo hold.

\subsubsection{Civilian Intercoms}

Civilian intercom systems started with the same headset specifications that military systems had. Because civilian intercom systems are not hindered by military standards, the intercoms evolved more quickly. Most notable of the additional features for civilian intercoms are the ability to mix in music to the intercom and have voice activation. These features and others give reason for choosing civilian intercoms when implementing a new intercom system.

\subsection{Headsets and their impedances}


The world of aircraft intercom headsets has evolved resulting in split standards throughout the years [5]. The end result is a standard for most commercial aircraft and a standard for all military aircraft.

Both the military and commercial speakers in headsets started out as $300 \mathrm{Ohm}$ speakers connected in series (Figure $6 \mathrm{C}$ ). This created a total impedance of $600 \mathrm{Ohm}$ for a single headset. Speakers can, over time, either short circuit creating a $300 \mathrm{Ohm}$ speaker in one ear that will be half as loud or break to open circuit, which renders the headset useless (both speakers output no sound).

The military solution (Figure $6 \mathrm{~A}$ ) was to connect the speakers in parallel so that if the coils in the speaker broke to open circuit, there would not be a total malfunction. Since, the majority of the time, the coils break to open circuit instead of shorting this created a fail-safe system for headset speakers. Now, military headsets are a $150 \mathrm{Ohm}$ headset (total of the two $300 \mathrm{Ohm}$ speakers connected in parallel) that is mono (both speakers output the same signal).

Civilian aircraft headsets took a different route (Figure 6 B) by splitting the two speakers for stereo (each speaker has a separate signal), which kept the impedance of each speaker at $300 \mathrm{Ohms}$. This gave civilian pilots the ability to listen to music in stereo and not be concerned with a total failure if one speaker should fail. For specifications on headsets see Appendix B. 


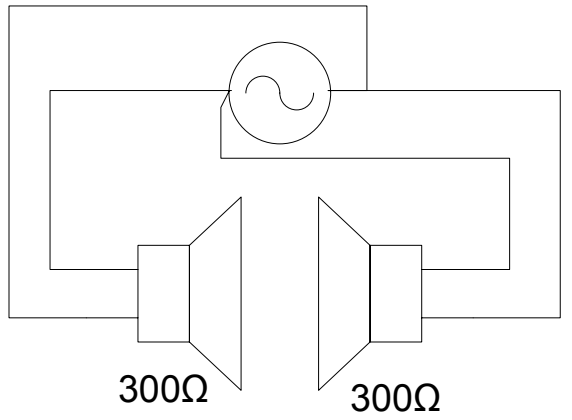

A) Military Standard, Parallel connected, Total Impedance $150 \Omega$

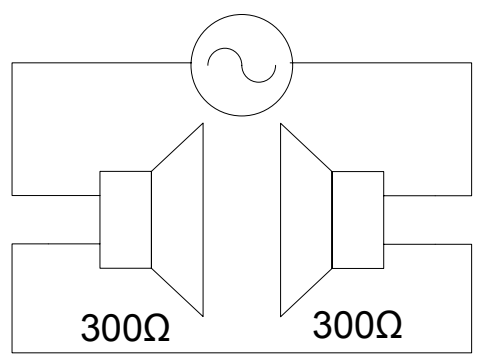

C) Older Military Standard, Series connected,

Total Impedance $600 \Omega$
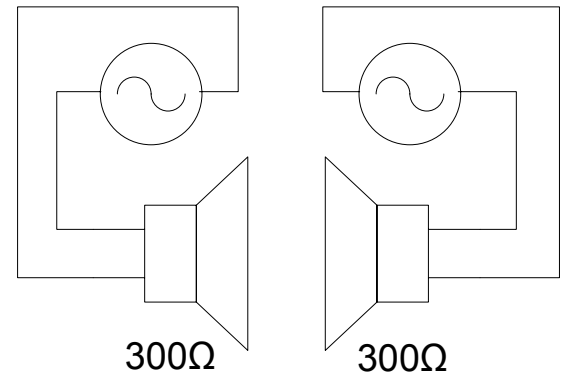

B) Civil. Stereo Standard, Independent connection, Total Impedance $300 \Omega$ Per Speaker

Figure 6 - Various Headset Speaker configurations 


\subsection{Microphones}

The microphones in headsets are also different in civilian and military aircraft.

The military continues to use dynamic microphones despite the advances of newer technologies. On the other hand, somewhere along the way civilian headsets switched to electret condenser microphones

Dynamic microphones (Figure 7) are nothing more than a tiny sensitive electro magnet. Dynamic microphones contain a film that moves in a cone and induces a voltage in the coil that is very low, about $2.5 \mathrm{mVrms}$ maximum, while the impedance of the dynamic microphone is about 5-ohms, also very low.

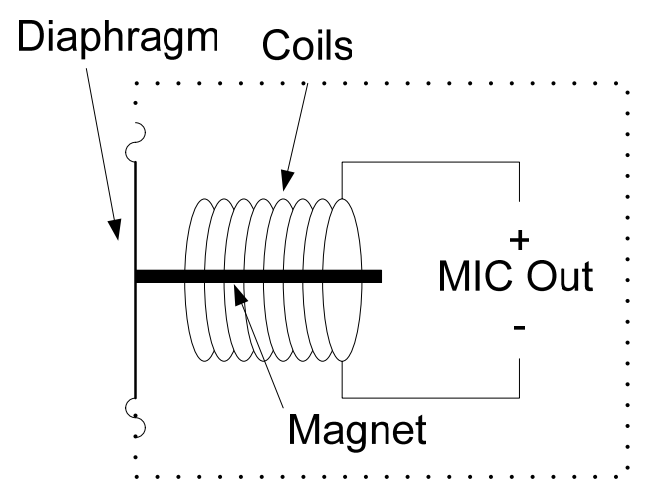

Figure 7 - Dynamic Microphone 
Electrect microphones (Figure 8) contain a capacitive plate that moves, changing the voltage across it resulting in a signal. Electret microphones deliver an AC signal of about 500mVrms, from a built-in operational amplifier, and have large impedances, about $1 \mathrm{k}-\mathrm{ohm}$. Because an electret microphone needs to have the capacitive plate biased, a 3-9 volts DC source is needed.

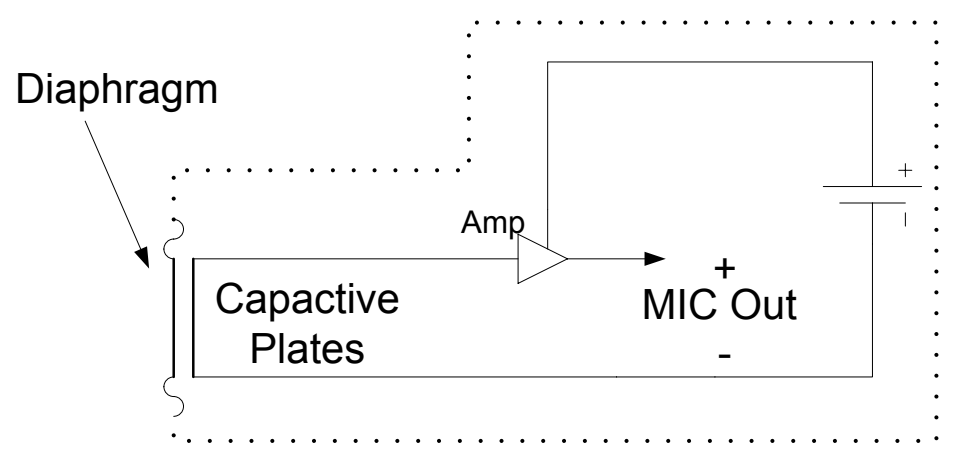

Figure 8 - Electret Condenser Microphone

The advantage of the electret over the dynamic microphone is that the electret does not require very much amplification at its output end because it includes an integral operational amplifier (Figure 8) inside the microphone. Thus, the electret is less subject to interference on the signal wires because of the higher signal levels. Long transmission lines introduce voltage noise on the electrect microphone, while the dynamic microphone is mainly driving current and is well suited for long wires. 
Project Oculus provides a set of commercial FlightCom Denali ANR headsets

(Figure 9). These were purchased for use in the first operator station. These headsets contain Active Noise Reduction (ANR) circuits, which further reduce audio noise inside the headset. Flightcom also makes an ANR headset for the military that meets their specifications. Figure 10 shows the military headset for comparison to the civilian headset. The main difference between the two headsets is the microphone type, the mono and stereo speakers, and the connectors.

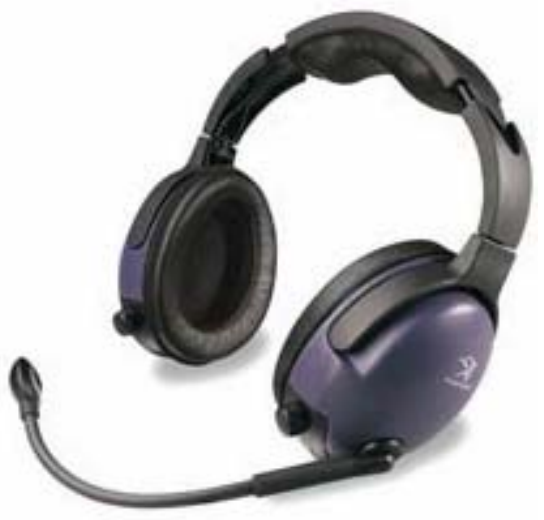

Figure 9 - Flightcom Denali ANR

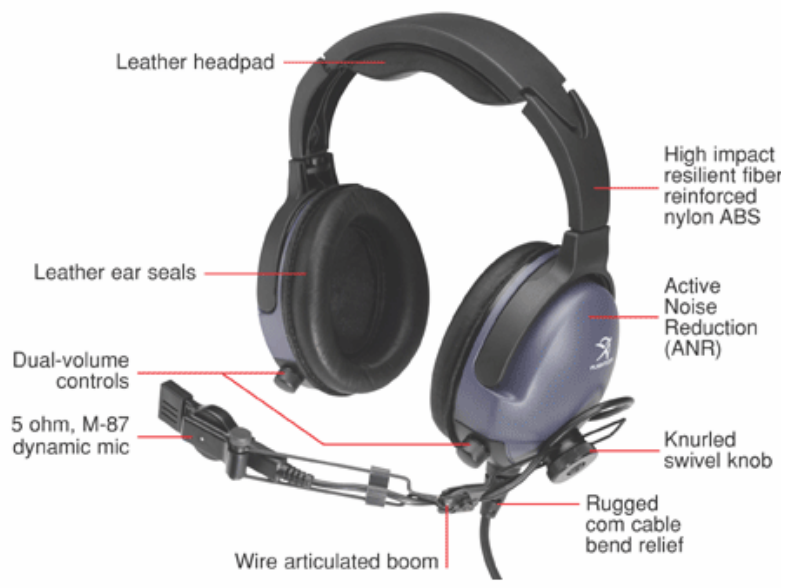

Figure 10 - Flightcom Denali ANR Military 


\section{Chapter 4. Intercom Design for Project Oculus}

The intercom designed for Project Oculus must be able to support three people inside the operator station, one person outside the operator station, and be able to communicate with the C-130 airplane's intercom system. The Project Oculus intercom must also support whatever headset type the operator chooses to use. Supporting different headsets is a difficult task mainly because available intercom control boxes support only one specific type of headset.

The requirement that military and civilian headsets be supported in the intercom system is addressed by an impedance matching device designed specifically for this project. Inside the impedance matching device is an amplifier that allows for proper power to be transferred to the headset speaker and a microphone amplification circuit that allows the dynamic microphone to be used with civilian intercom control devices.

Military personnel or others will be using Project Oculus to perform a wide variety of missions and need wide support and simple upgradeability. The requirements of the intercom system for Project Oculus are to provide an expandable system that allows for future upgrades, allow for communications with the intercom system on the C130 aircraft, support three operator positions, and support one external headset connection.

\subsection{Intercom System}

The core of the intercom system designed here is the Northern Airborne Technology (NAT) AA83 intercom control unit. This intercom control unit is a standard civilian intercom that provides many quality features such as voice activation, tie line, radio support, and communication isolation from other intercom control units. This 
device connects to the headsets, music inputs, and the tie line. Component devices such as the NAT digital crossover device (AA36-100), allow the NAT AA83 to have proper signals that can transmit and receive audio to the C-130 intercom system.

The concept of a device to support headsets that contain speakers with different impedances and different types of microphones is shown in Figure 11. This device should pass the speaker signals from the intercom control box to the headset making sure that the entire signal in its original form arrives at the speaker. This means that if the impedance of the headset does not match what the intercom control requires it will have to change the impedance. Also, the device should convert the microphone signal level to that of the proper signal level for the intercom control box. 


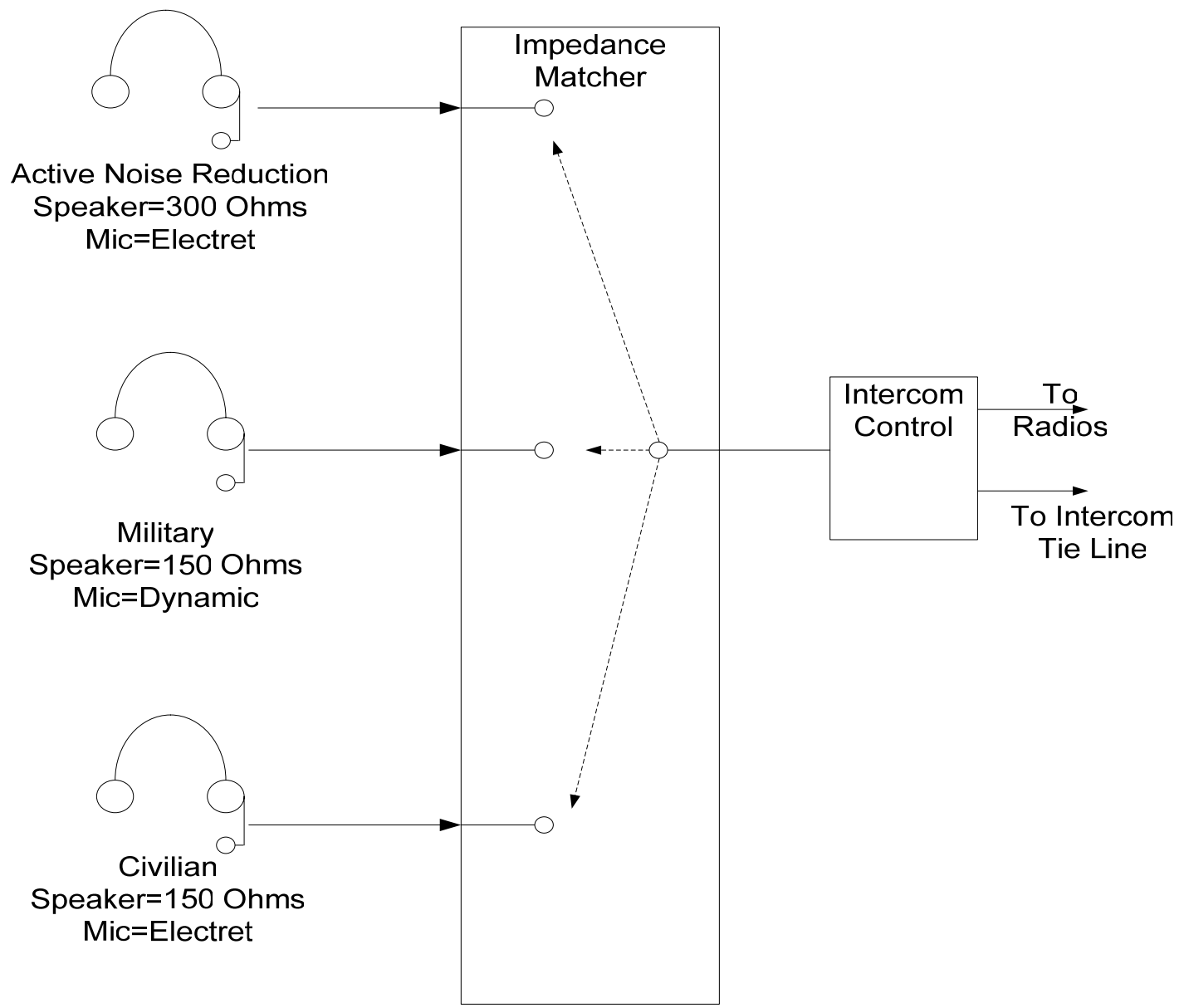

Figure 11 - Concept Headset Selection Device per Operator Station

Testing the audio sound level of the headsets, see 4.3.2, demonstrated that when the $150 \mathrm{Ohm}$ headsets and $300 \mathrm{Ohms}$ headsets were connected directly to the NAT intercom control device the $150 \mathrm{Ohm}$ headsets have a volume that is lower than that of the $300 \mathrm{Ohm}$ headsets. Further testing with the impedance matching device, 4.3.3, shows that when using the impedance matching device the 150 Ohm headsets become louder near the level of the $300 \mathrm{Ohm}$ headset driven directly from the NAT intercom control box for which it was originally designed.

Amplifying the dynamic microphone of the military headset was achieved by using the National Semiconductor LM386 operational amplifier. Recall that a dynamic microphone gives a $2.5 \mathrm{mVrms}$ level signal and the NAT intercom control device requires 
500mVrms levels. The LM386 amplifier device has a built in ability to provide the necessary amplification internally with minimal external parts. Testing of this device, see 4.3.5, shows that proper signal levels were reached. 


\subsection{Intercom Design}

The custom built impedance matching device will allow the use of different headset types that are not compatible with the AA83 intercom control device. This allows aviators that carry their own headsets with them to use what they feel most comfortable with. The importance of the device to pass voice frequencies to the headsets was a major design goal.

The impedance matching device consists of three amplifier circuits. The first amplifier has a voltage gain of 200, and is used for the amplification of the dynamic microphone signal as described previously. The other two amplifiers are used to buffer the signal to the headsets that are not impedance matched for the AA83 intercom control device. For more information on the design and construction of this device see Appendix A.

The intercom designed for Project Oculus provides for three different types of headset connections for each operator position inside the operator station, an external connection for a person operating outside the operator station, and another external cable connection to provide communications with the $\mathrm{C}-130$ intercom system. A basic connection diagram is shown by Figure 12 below. The details and schematics are discussed in Appendix B. 

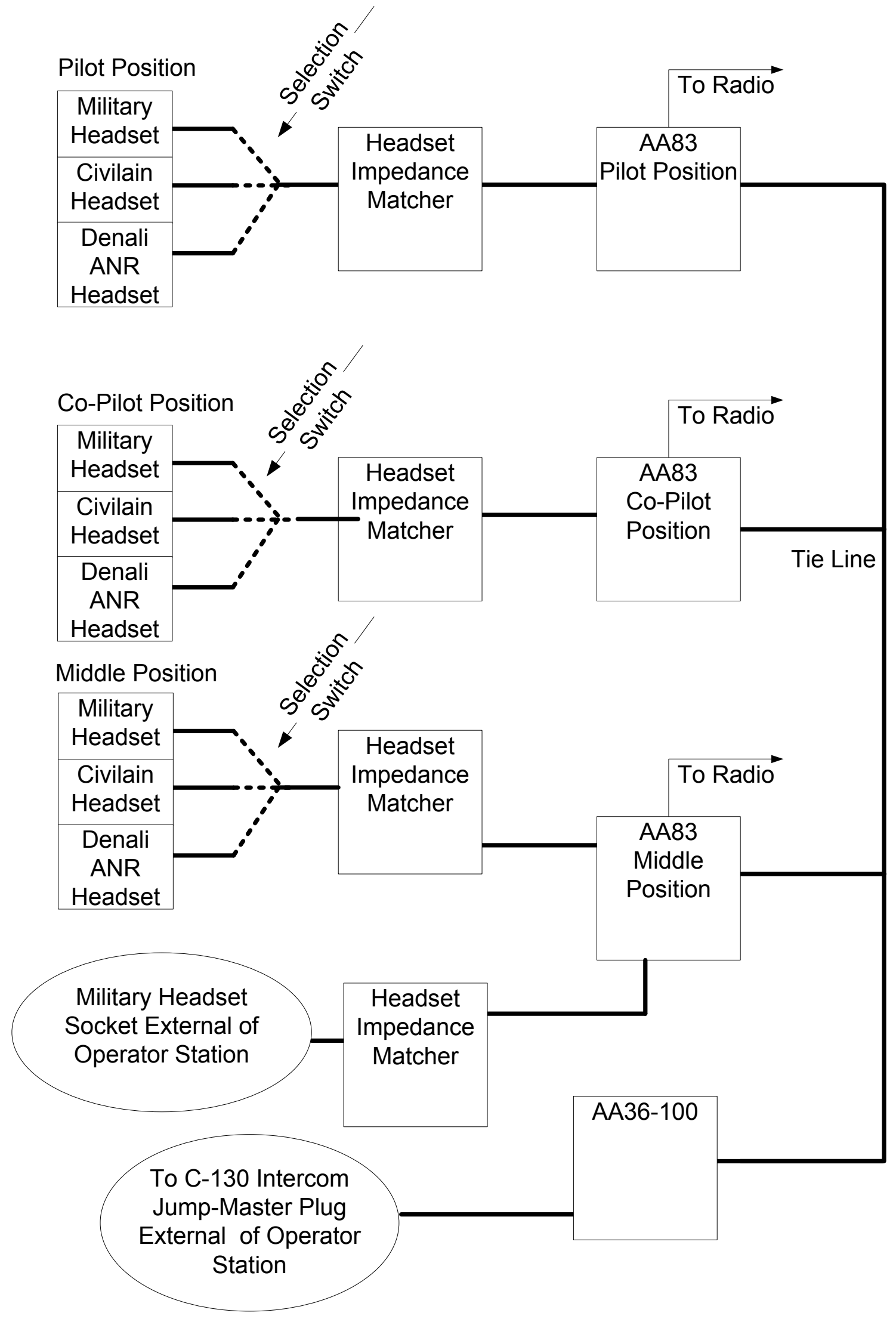

Figure 12 - Basic Block Diagram of Intercom Connections 


\subsection{Intercom Testing}

This section is concerned with various measurements preformed on the intercom system to verify operation of the impedance matching box. The tests include 1) frequency responses of the intercom system, including the NAT AA83 and the impedance matching box with different headset loads, 2) frequency response of sound output of the headsets, and 3) dynamic microphone amplification testing. Figure 13 shows an overall view of the tests preformed where the lowest level is an actual measurement.

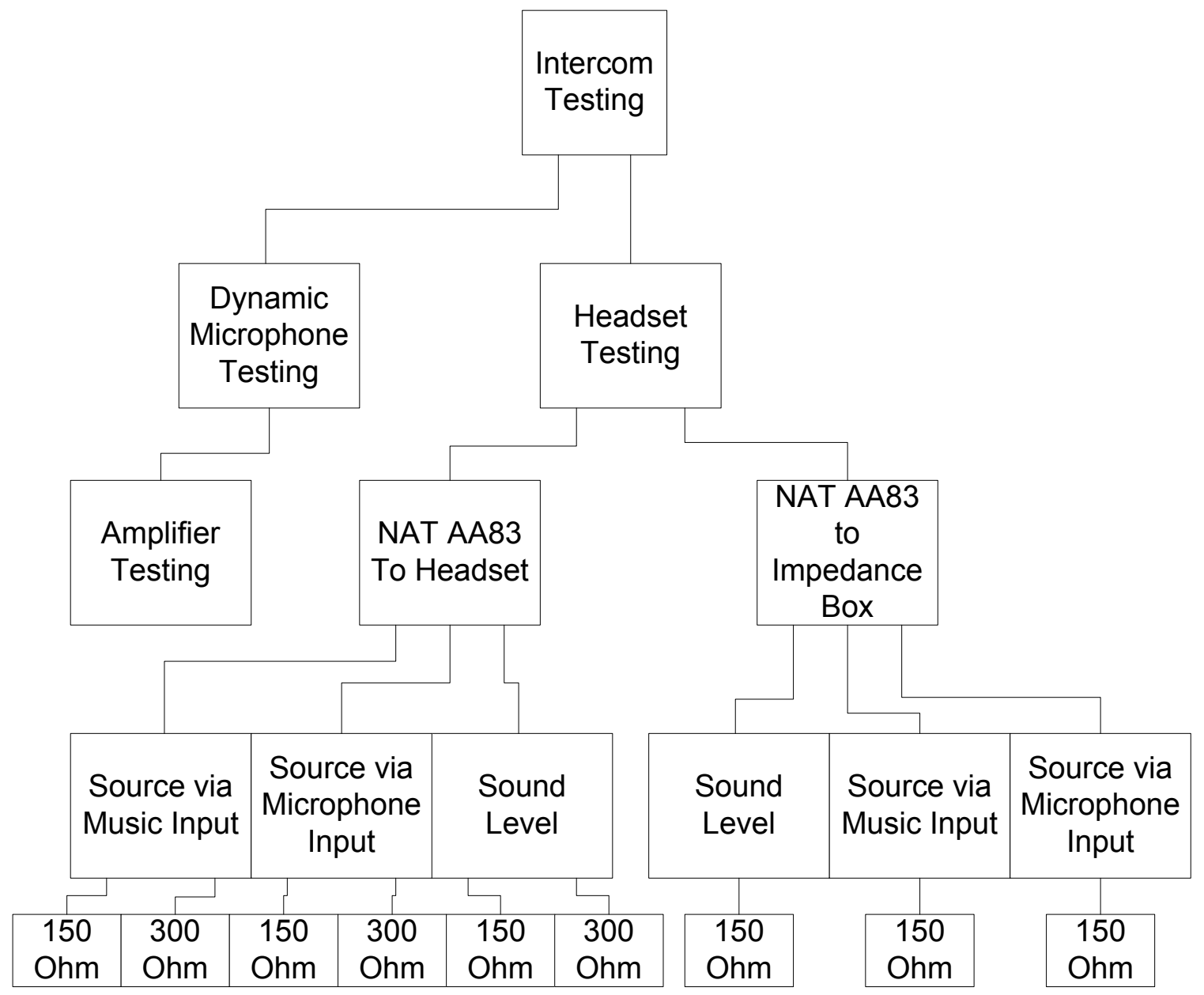

Figure 13 - Intercom Testing Tree

The source signal generator used was a Tektronix CFG253 which is shown to have a flat voltage versus frequency for its output for loads that match its internal 
impedance. The output impedance of the signal generator is specified at $50 \mathrm{Ohms}$. The oscilloscope probe used in the tests, Tektronix TDS3052B oscilloscope, is a high impedance voltage probe. All of the tests involving the AA83 were conducted with the volume control knob set at half the full level unless otherwise stated.

\subsubsection{Methods of Calculation}

The majority of the graphs show levels of either voltage gain in $\mathrm{dB}, \mathrm{dBm}$ or $\mathrm{dBuV}$. A dB, or Decibel, is a logarithmic scale that allows humans to deal with large dynamic range numbers instead of extremely large or extremely small numbers. A decibel refers to a relative change, such as a voltage gain (voltage out divided by voltage in). This can also assume "voltage in" to be a standard of one volt (dBV), or one microvolt $(\mathrm{dB} \mu \mathrm{V})$.

Voltage gain in terms of $\mathrm{dB}$ (i.e. referenced to one volt) is shown in equation (1). Another type of decibel calculation is the dBV (decibel volt), which is nothing more than a decibel with reference to one volt, as shown in equation (2). The calculation of power in $\mathrm{dBm}$, or $\mathrm{dB}$ milliwatts, is determined by ten times the log of the power in milliwatts, as shown in equation (3) with an input power of $1 \mathrm{~mW}$. 


$$
\begin{gathered}
\text { Gain }=\frac{P_{o}}{P_{i}}=\frac{V_{o} \cdot I}{V_{i} \cdot I}=\frac{\frac{V_{o}^{2}}{R}}{\frac{V_{i}^{2}}{R}}=\frac{V_{o}^{2}}{V_{i}^{2}} \\
d B=10 \cdot \log _{10}\left(\frac{V_{o}}{V_{i}}\right)^{2}=20 \cdot \log _{10}\left(\frac{V_{o}}{V_{i}}\right)=20 \cdot \log _{10}(\text { Gain })
\end{gathered}
$$

Decibel Gain, $d B=20 \cdot \log _{10}\left(\frac{V_{o}}{V_{i}}\right)$

Decibel Volt,$d B V=20 \cdot \log _{10}($ Voltage $)$

Decibel milliwatts, $d B m=10 \cdot \log _{10}\left(P_{\text {milliwatts }}\right)$

Power calculations used for testing were of two different types, real power and apparent power. Real power, or sometimes the average power, is a measure of how much heat is delivered to the real, or just resistive, components of a system. This measurement is calculated in Watts, as shown by equation 4, where V is the RMS (Root Mean Square) voltage across the system and $\mathrm{R}$ is the resistance, in Ohms, of the system. The calculation of apparent power consists of combining the real power and the complex reactive power. In this case speakers are involved, therefore inductance fills the role of reactance in the equation, shown by equation 5 . Reactance is a function of the inductors physical properties in Henries, represented by $\mathrm{L}$, and the frequency that the system is operating under, as shown by equation 6 . Apparent power is a combination of the real power and reactive power, which forms the unit VA (Volts-Amps), and is shown in equation 7. The 
foundation of apparent power, which consists of real power and complex power is demonstrated by Figure 14 - Power Triangle, which is the classical power triangle.

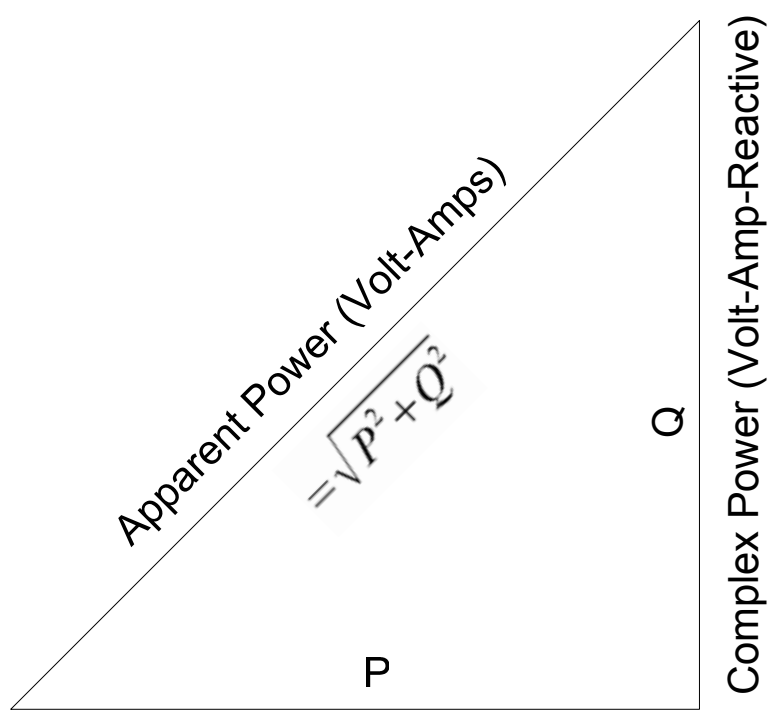

Real Power (Watts)

Figure 14 - Power Triangle

Real Power $=V_{d c} \cdot I_{d c}=V_{m m s} \cdot I_{m m s}=\frac{V_{m s}^{2}}{R}$ (Watts)

Reactive Power $=|Q|=\left|V_{m s} \cdot I_{m s}\right|=\left|\frac{V_{m s} \cdot V_{r m s}}{j X}\right|$

$$
|Q|=\left|\frac{V_{m s}^{2}}{X}\right|(\text { Volt-Amp-Reactive })
$$

Reactance of a Inductor $X=L \cdot 2 \cdot \pi \cdot f(\mathrm{Ohm})$

Apparent Power $=\sqrt{P^{2}+Q^{2}}$ (Volt-Amps) 


\subsubsection{Headset tests}

The voltage gain versus frequency tests, $20 \mathrm{~Hz}$ to $20 \mathrm{kHz}$, of the headset are broken down into several categories. First, testing of the NAT AA83 intercom device with the 150 and $300 \mathrm{ohm}$ headsets connected directly to the proper device outputs. The NAT AA83 has three connections for inputs, microphone, music, and the tie line. All three or one of the inputs can be used simultaneously, but for the following tests only the microphone input or the music input was used. Second, measurements were taken with the impedance matching device driving the $150 \mathrm{Ohm}$ headset to determine how well it performs. Third, a sound dB meter, type RadioShack 33-2055, was used to measure the sound ouput in terms of sound pressure level (SPL) of the headsets.

The Military specification headset, which generally has a speaker impedance of $150 \mathrm{Ohms}$, was used as the $150 \mathrm{Ohm}$ test headset. The mono civilian and military headsets have the same speaker specification, so only one type of these is required. The Flightcom Denali ANR headsets are 300 Ohm stereo speakers. Both speakers of the Denali headset were connected in stereo mode. Only the left channel was used for voltage measurements, except during the sound level tests where stereo mode was utilized.

\subsubsection{AA83 Frequency Response Using the Microphone Input}

This testing section is devoted to testing different headset impedances while using the microphone input of the NAT AA83. The AA83 has an input that is specifically designed for electret microphone signal level, know as the microphone input. This connection, one of many, was used as an input to the system for these tests. The signal generator impedance $(50 \mathrm{Ohm})$ did not meet the impedance specifications that the AA83 
requires for a microphone input $(150 \mathrm{Ohm})$, so the source impedance of the signal generator was raised with a series resistor $(100 \mathrm{Ohm})$. A diagram of this test is shown in

Figure 15. The volume knob on the AA83 remained in the half position during all testing. The input voltage levels varied from $125 \mathrm{mVrms}$ to $500 \mathrm{mVrms}$, which is the specified range in the AA83 specifications. The voltage output of the signal generator is show in Figure 16, and is flat for all tests. Voltage output from the AA83 to the headsets is shown in Figure 17. Voltage gain of the AA83 output from the signal generator is shown in Figure 18. Apparent power from the two input levels is shown in Figure $19 \&$ Figure 20.

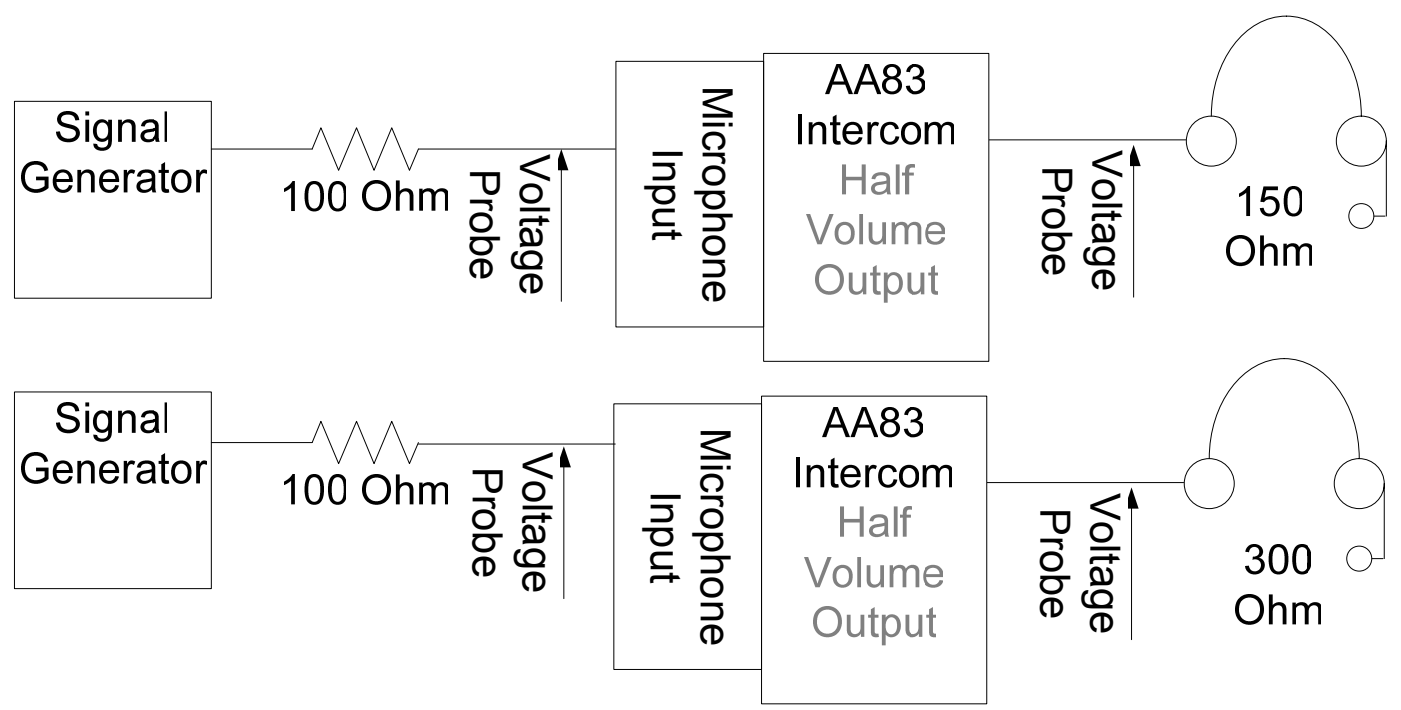

Figure 15 - AA83 Intercom Test Setup using Microphone Input 


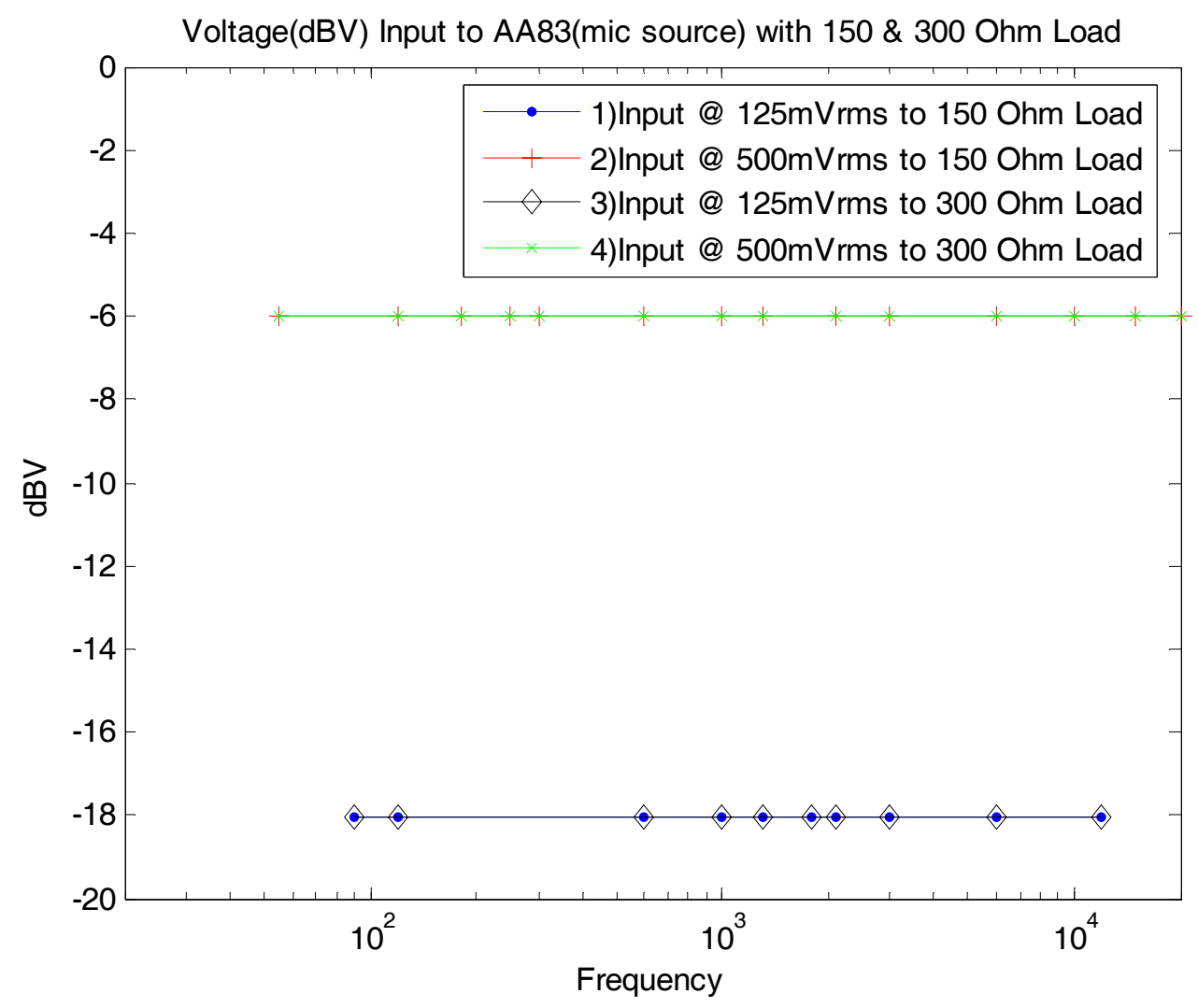

Figure 16 - Voltage vs Frequency AA83 Microphone Input

Voltage(dBV) Output of AA83(mic source) with $150 \& 300$ Ohm Load

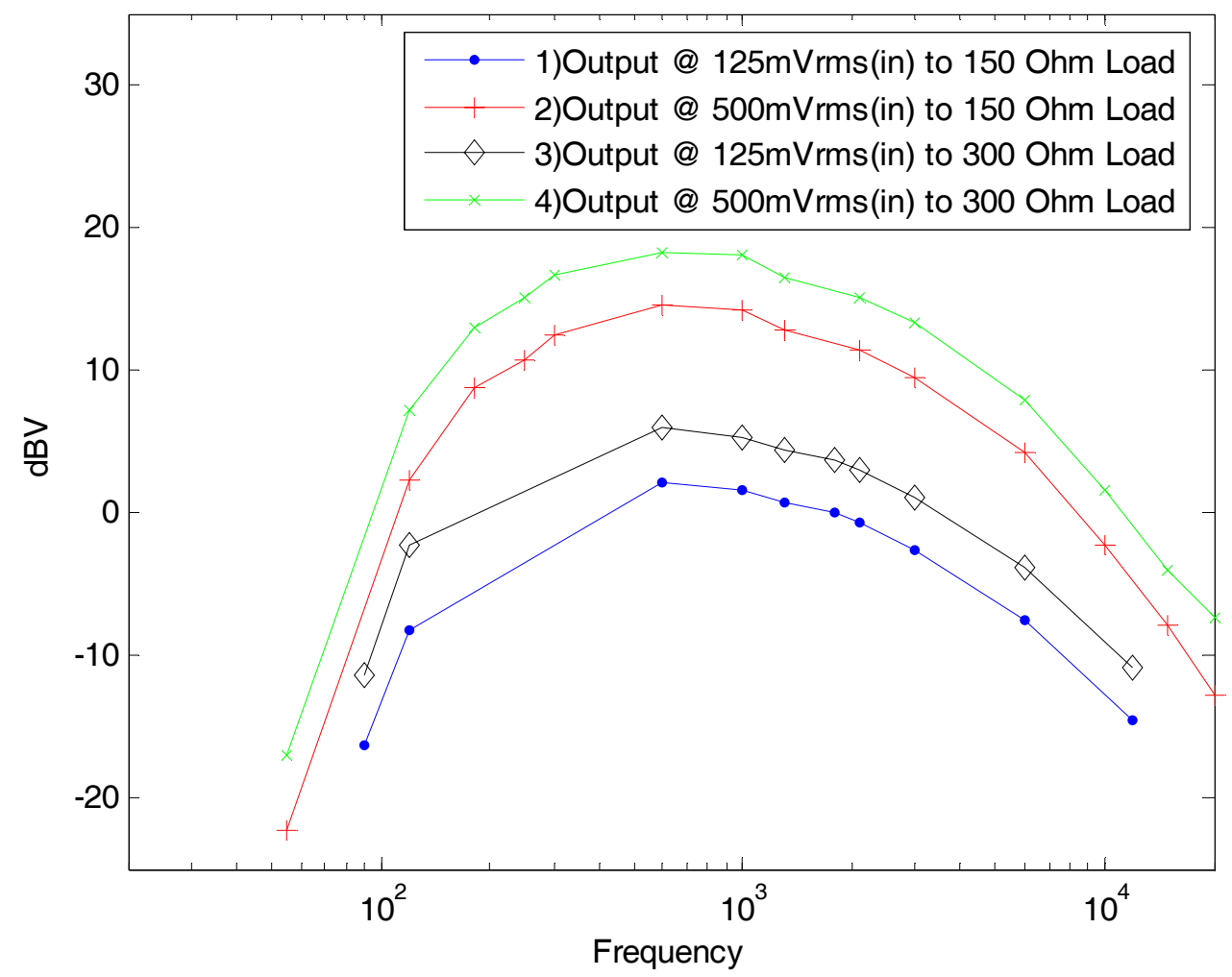

Figure 17 - Frequency Response of AA83 using 150 \& $300 \Omega$ Speaker Loads 
Voltage Gain(dB) of AA83(mic source) with 150 \& 300 Ohm Load

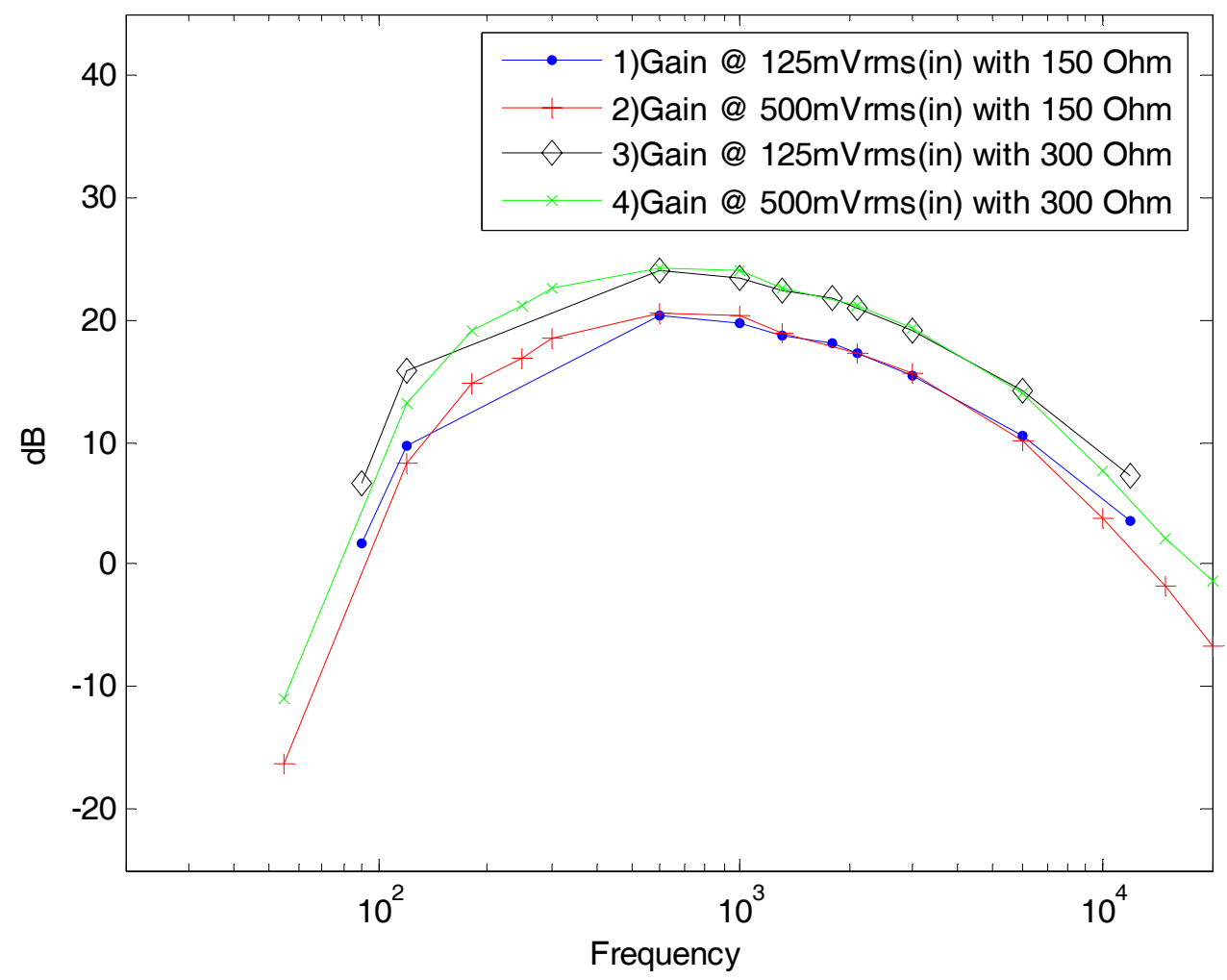

Figure 18 - Calculated Voltage Gain of AA83 with 150 \& $300 \Omega$ Speaker Loads Apparent Power output from AA83(mic source) to $300 \& 150 \mathrm{Ohm}$

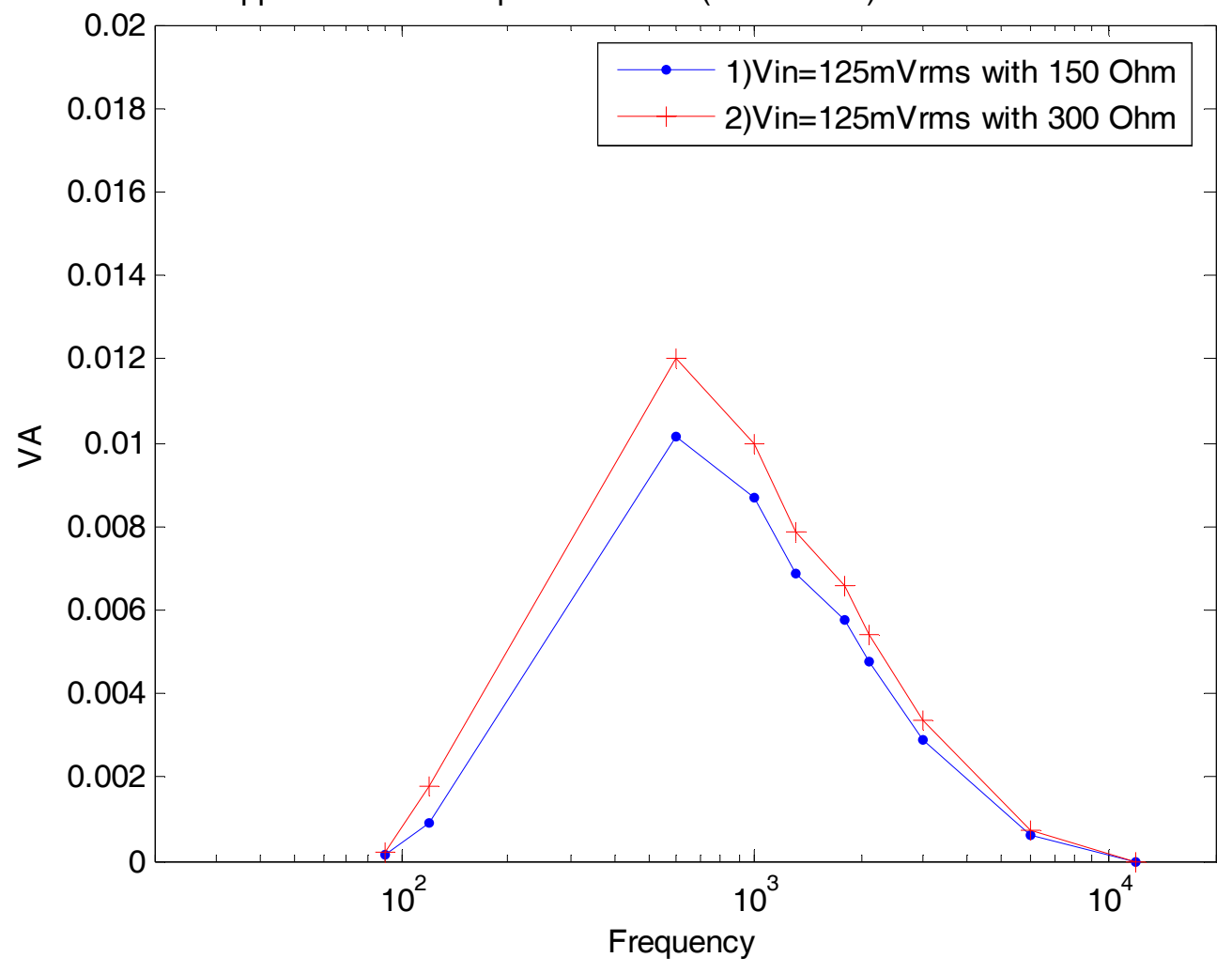

Figure 19 - Calculated Apparent Power@125mVrms to 150 \& $300 \Omega$ Speaker Loads 


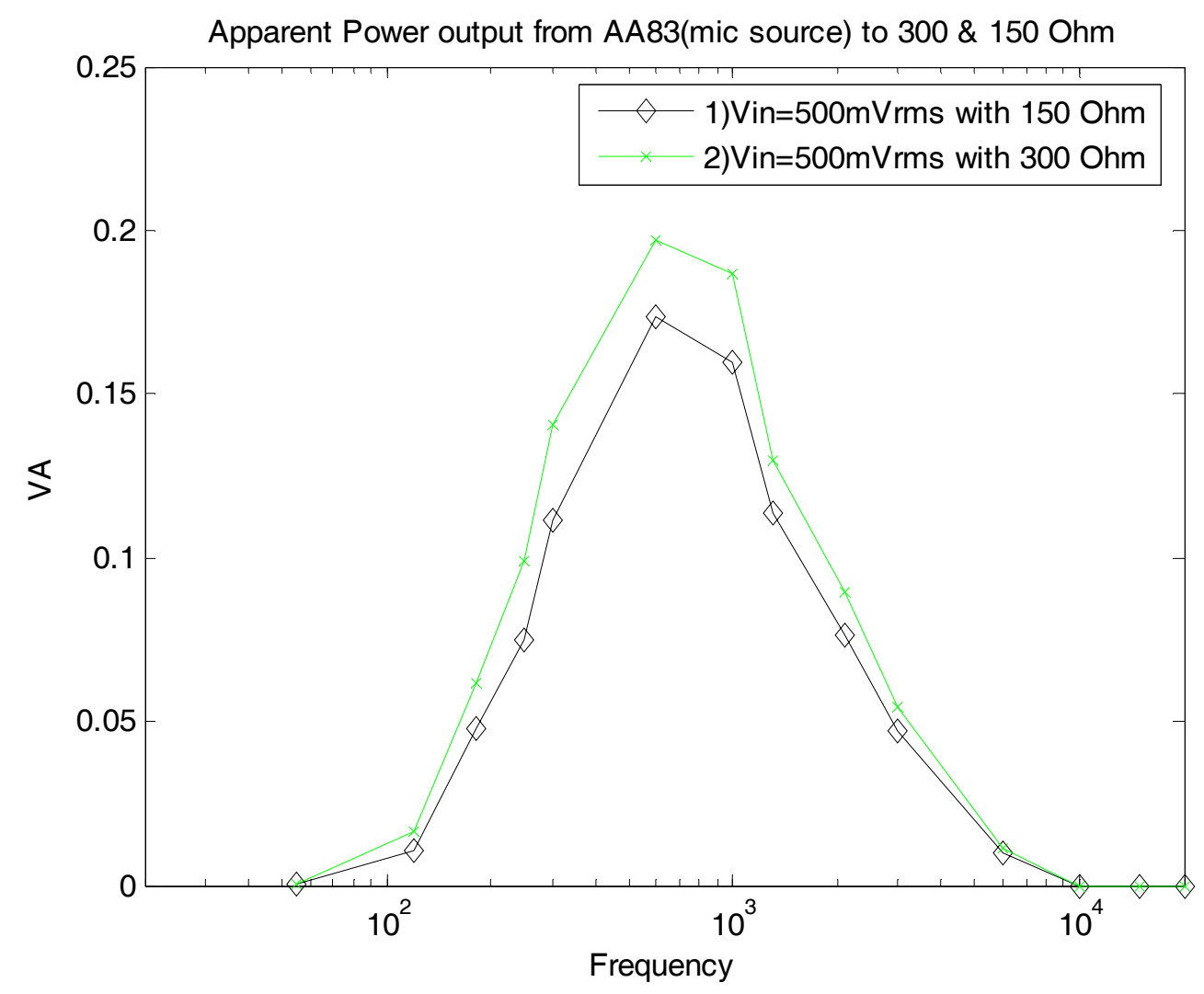

Figure 20 - Calculated Apparent Power@ 500mVrms using 150 \& $300 \Omega$ Speaker Loads

\subsubsection{AA83 Frequency Response Using the Music Input}

This testing section is devoted to testing different headset impedances while using the music input of the NAT AA83. The AA83 has an input that is specifically designed for common music device level inputs. These signal levels are about 1 Vrms, and are usually stereo, but for this test only one channel was used. The music source was used as an input to the system for these tests. The signal generator did not meet the impedance specifications that the AA83 requires for a microphone input, so this was balanced with a series resistor of value $10 \mathrm{kOhm}$. The volume knob on the AA83 remained in the half position during all testing. The setup of the tests is shown in Figure 21 .The input voltage levels varied from $900 \mathrm{mVrms}$ to $1.5 \mathrm{Vrms}$, which is the specified range in the AA83 specifications for a music input. The voltage output of the signal generator is shown by 
Figure 22, and is flat for all tests. The voltage output from the AA83 to the headsets is shown in Figure 23Figure 17. Voltage gain of the AA83 output from the signal generator is shown in Figure 24. Apparent power from the two input levels is shown in Figure 25 \& Figure 26.

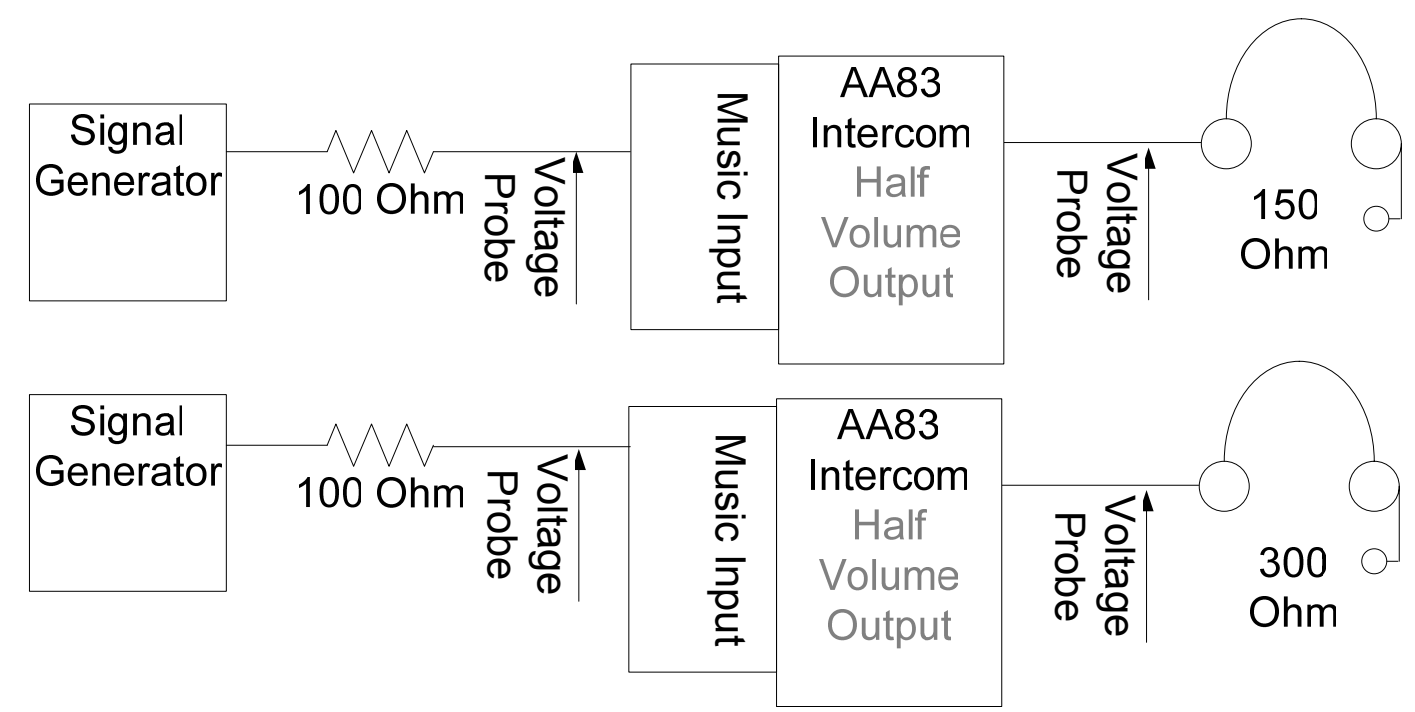

Figure 21 - AA83 Intercom test Setup using Music Input

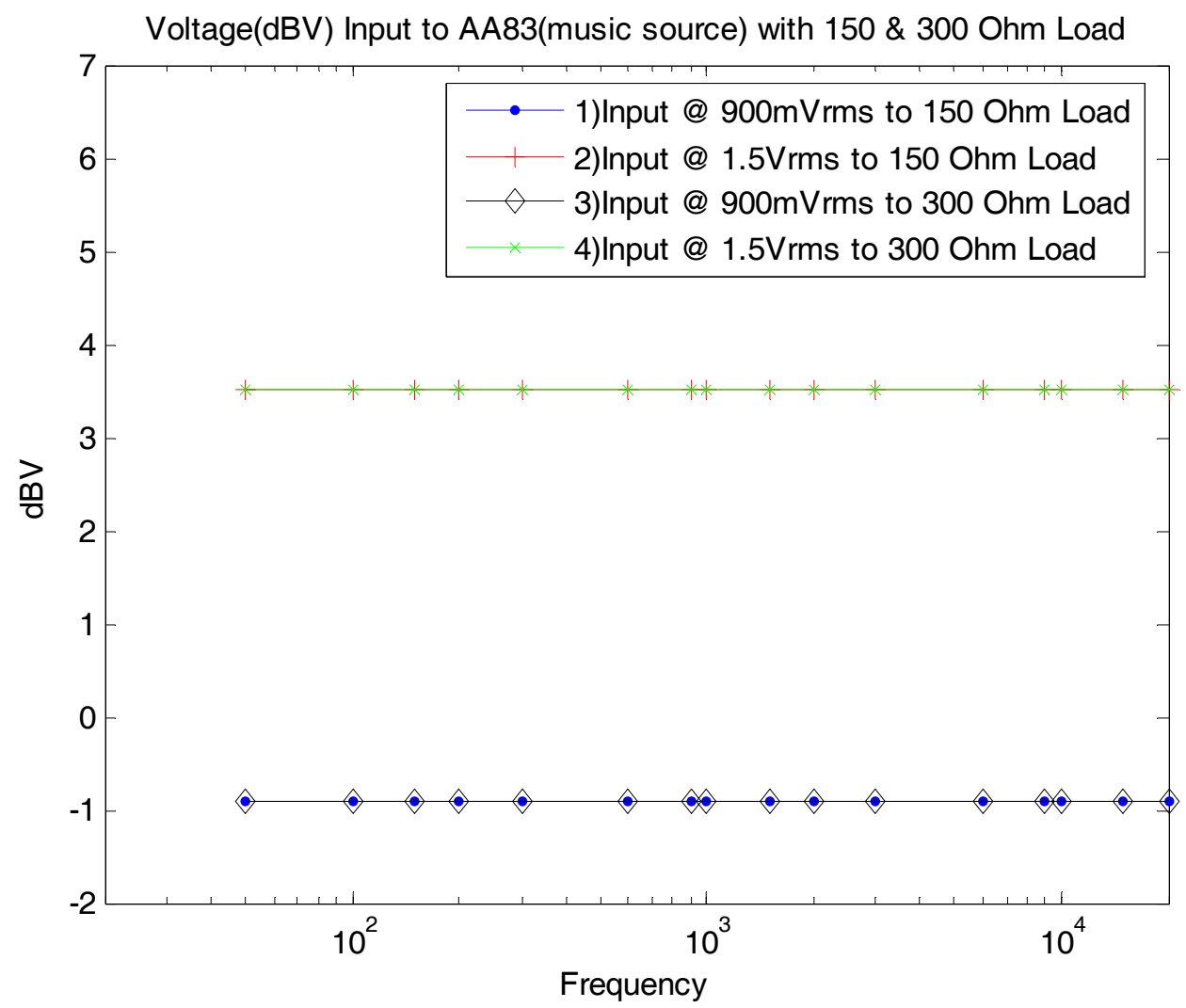

Figure 22 - Voltage vs Frequency to AA83 Music Input 


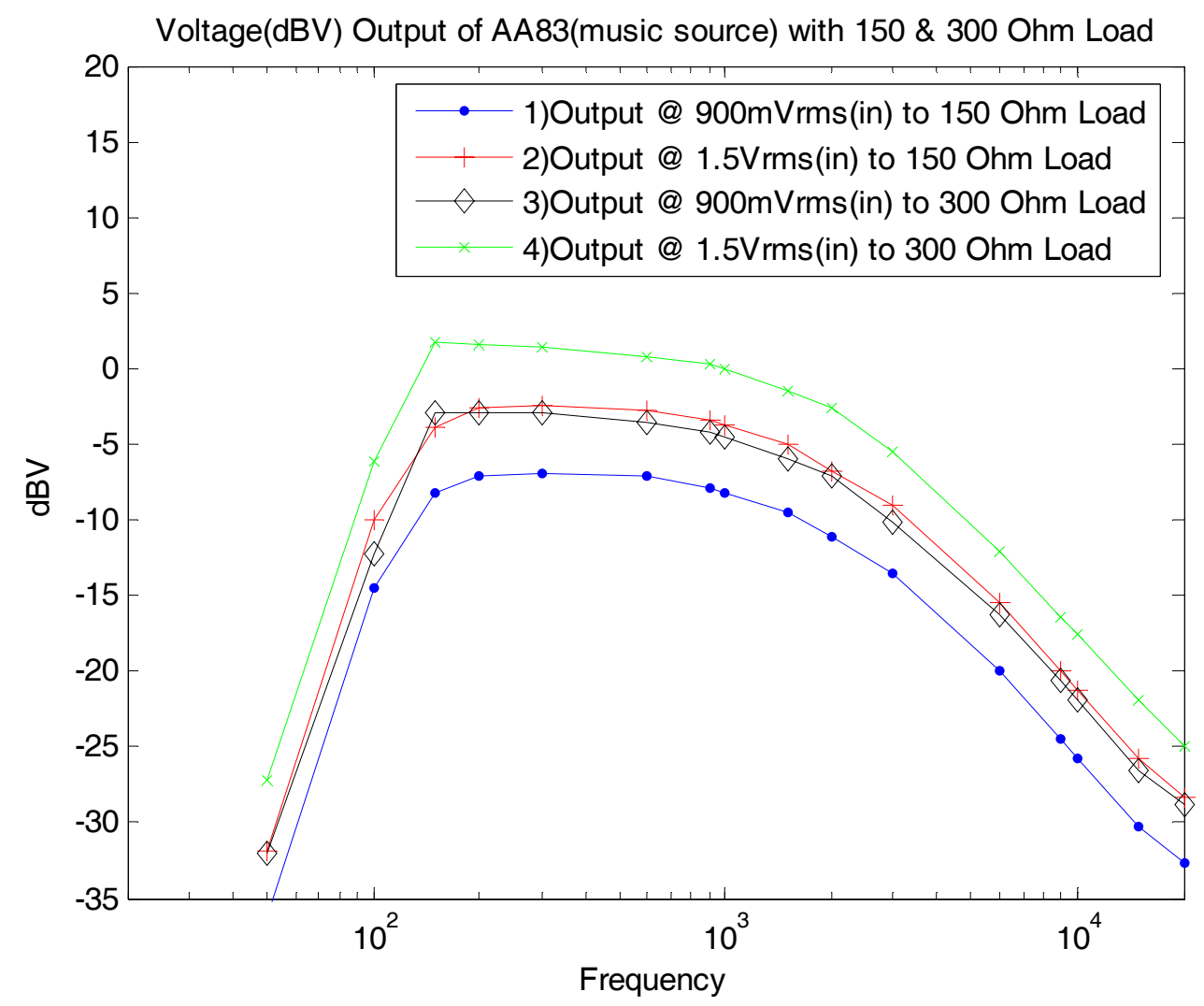

Figure 23 - Frequency Response of AA83 using 150 \& $300 \Omega$ Speaker Loads Voltage Gain(dB) of AA83(music source) with 150 \& 300 Ohm Load

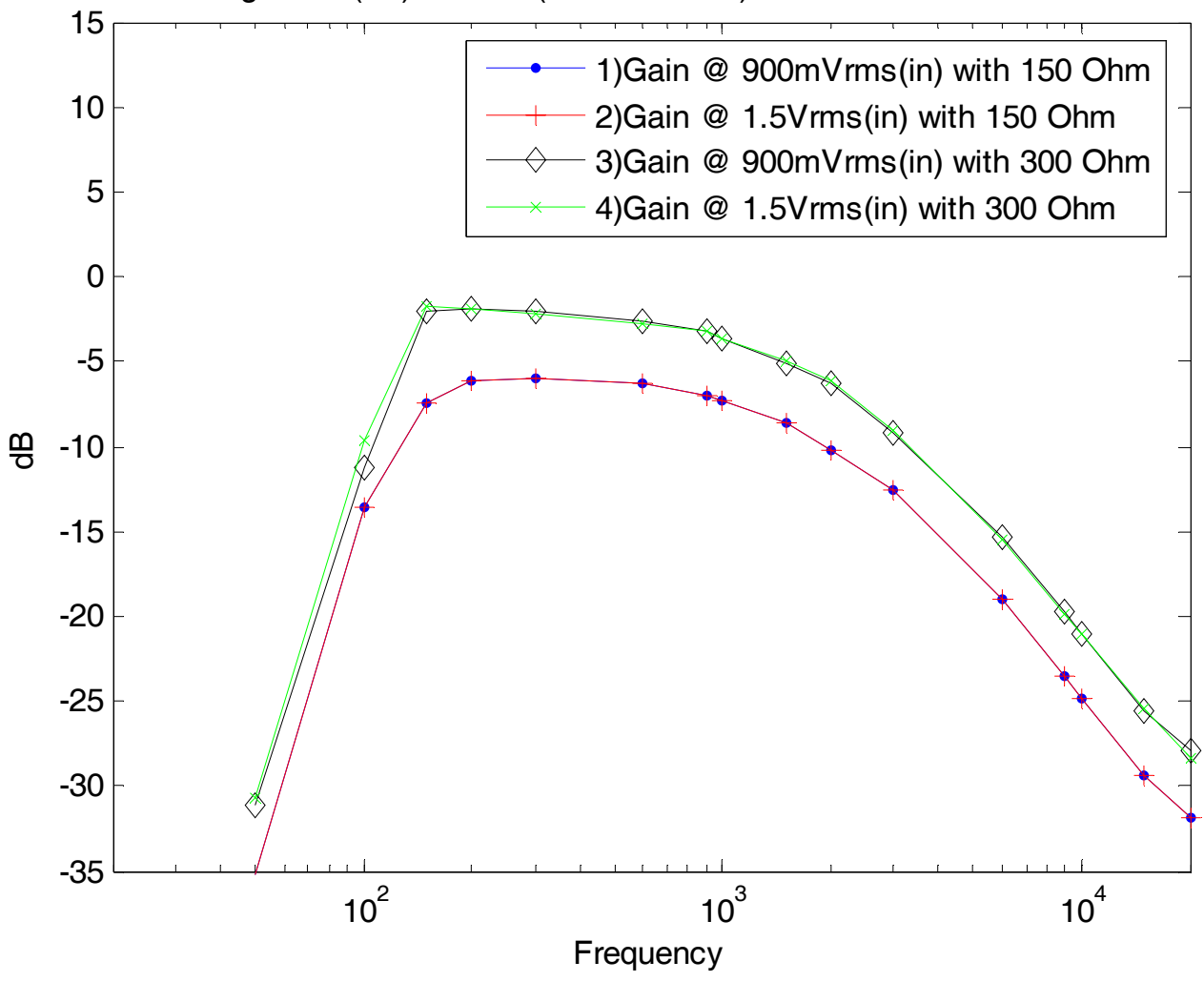

Figure 24 - Calculated Voltage Gain of AA83 using 150 \& $300 \Omega$ Speaker Loads 


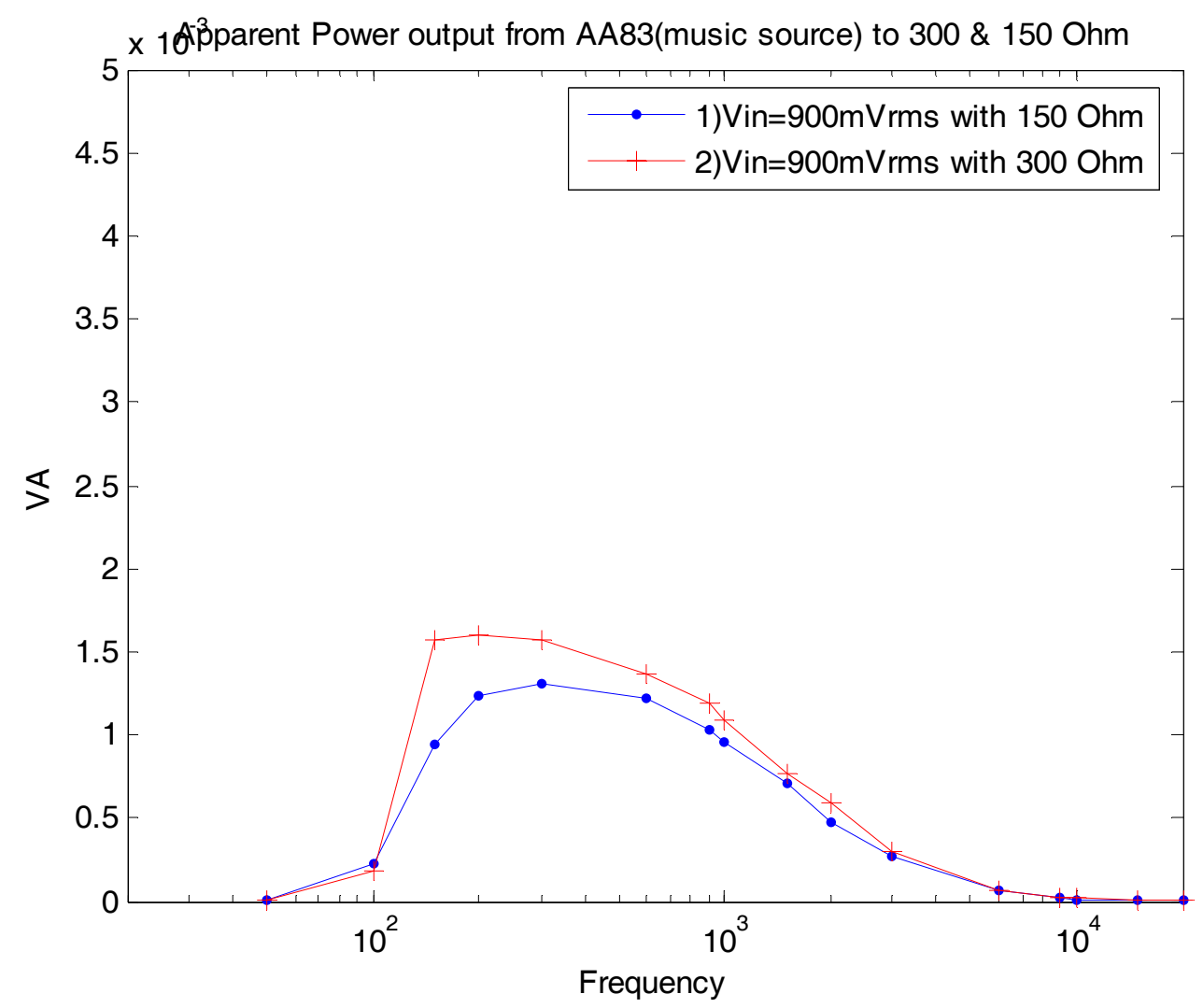

Figure 25 - Calculated Apparent Power@ 900 mVrms using 150 \& $300 \Omega$ Speaker Loads

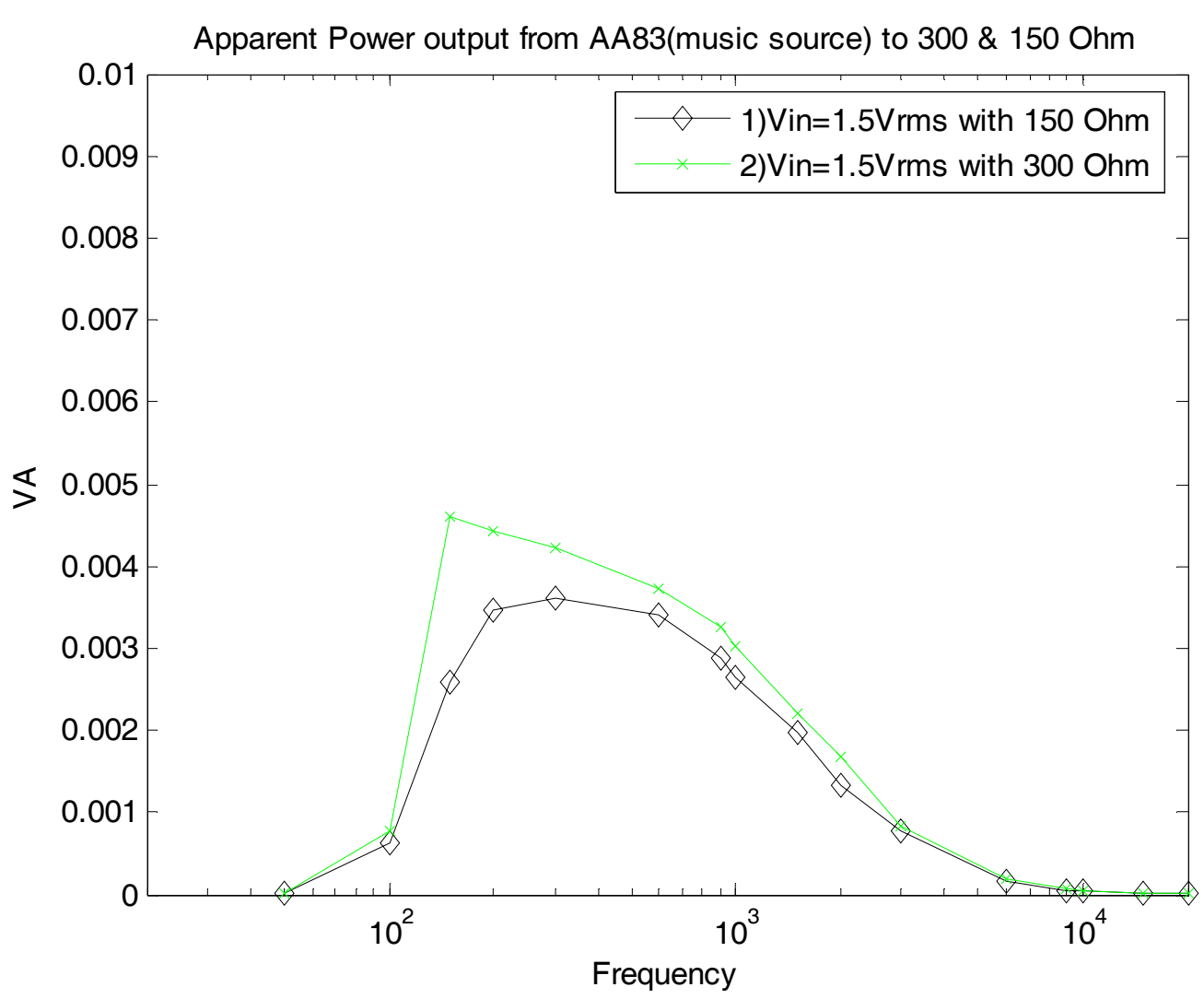

Figure 26 - Calculated Apparent Power @ 1.5 Vrms using 150 \& $300 \Omega$ Speaker Loads 


\subsubsection{Directly Driving Headsets from AA83 Conclusion}

The previous tests show two major properties of the NAT AA83. First, the inputs for both the microphone and music have a bandpass filter that is centered around the human voice range ( $300 \mathrm{~Hz}-3 \mathrm{kHz}$ ). Figure 23 demonstrates that the highest levels coming out of the AA83 occur around the human voice range. Second, the $150 \mathrm{Ohm}$ headset does not receive as much power as the $300 \mathrm{Ohm}$ headset, which is to be expected because the AA83 is designed for a $300 \mathrm{Ohm}$ load.

\subsubsection{Impedance Matching Circuitry Tests}

Testing the impedance box involved three tests. First, the impedance matching box was connected to the AA83, while the mic input was connected to the signal generator, as shown by Figure 27, and various measurements were taken. Second, the impedance matching box was connected to the AA83 while using the music input, shown by Figure 34, and various measurements were taken. Third the impedance box was subjected to different voltage level frequency sweeps to determine the response of the impedance box by itself.

The input voltage to the AA83 using the microphone input is shown in Figure 28 and the music input is shown in Figure 35. Voltage output from the AA83 and input to the impedance box with the microphone input can be shown by Figure 29 and music input by Figure 36. Voltage output from the impedance box with the $150 \mathrm{Ohm}$ headset load using the AA83 microphone input is shown by Figure 30 and the music input is shown by Figure 37. Voltage gain of the AA83, impedance box, and overall through the AA83 and impedance box with the AA83 microphone input is shown by Figure 31 and the music input is shown by Figure 38. Apparent power from the $150 \mathrm{Ohm}$ headset when the AA83 was using the microphone input is shown by Figure 32 and Figure 33. 
Measurements were taken with the signal generator, at different voltage levels, connected to the impedance box to determine the frequency response of the impedance box alone. The test setup is shown by Figure 41 . Voltage delivered by the signal generator is shown by Figure 42, output voltage is shown in Figure 43, and voltage gain is shown in Figure 44. Apparent power is shown by Figure 45.

\subsubsection{Impedance Matching Box Test with Microphone input to AA83}

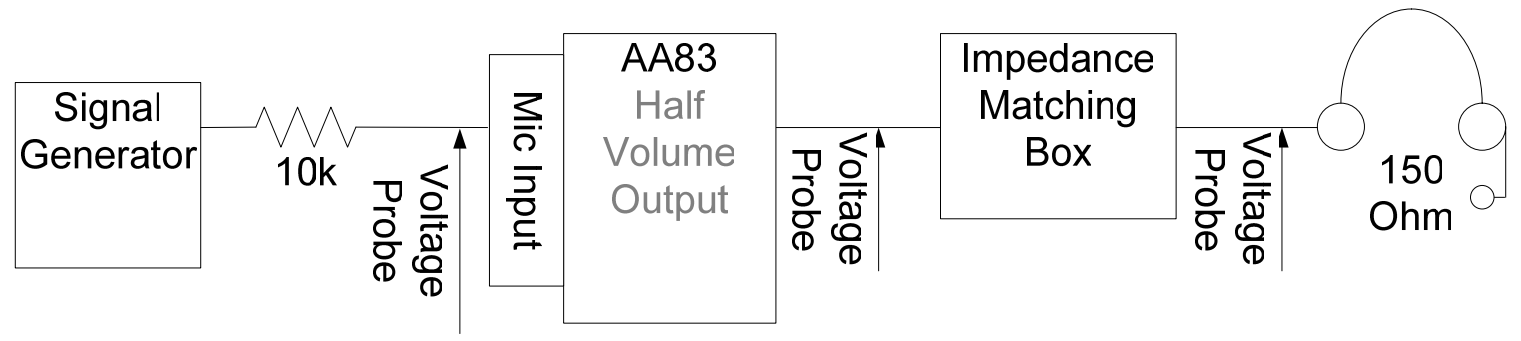

Figure 27 - Impedance box testing setup with AA83 mic input

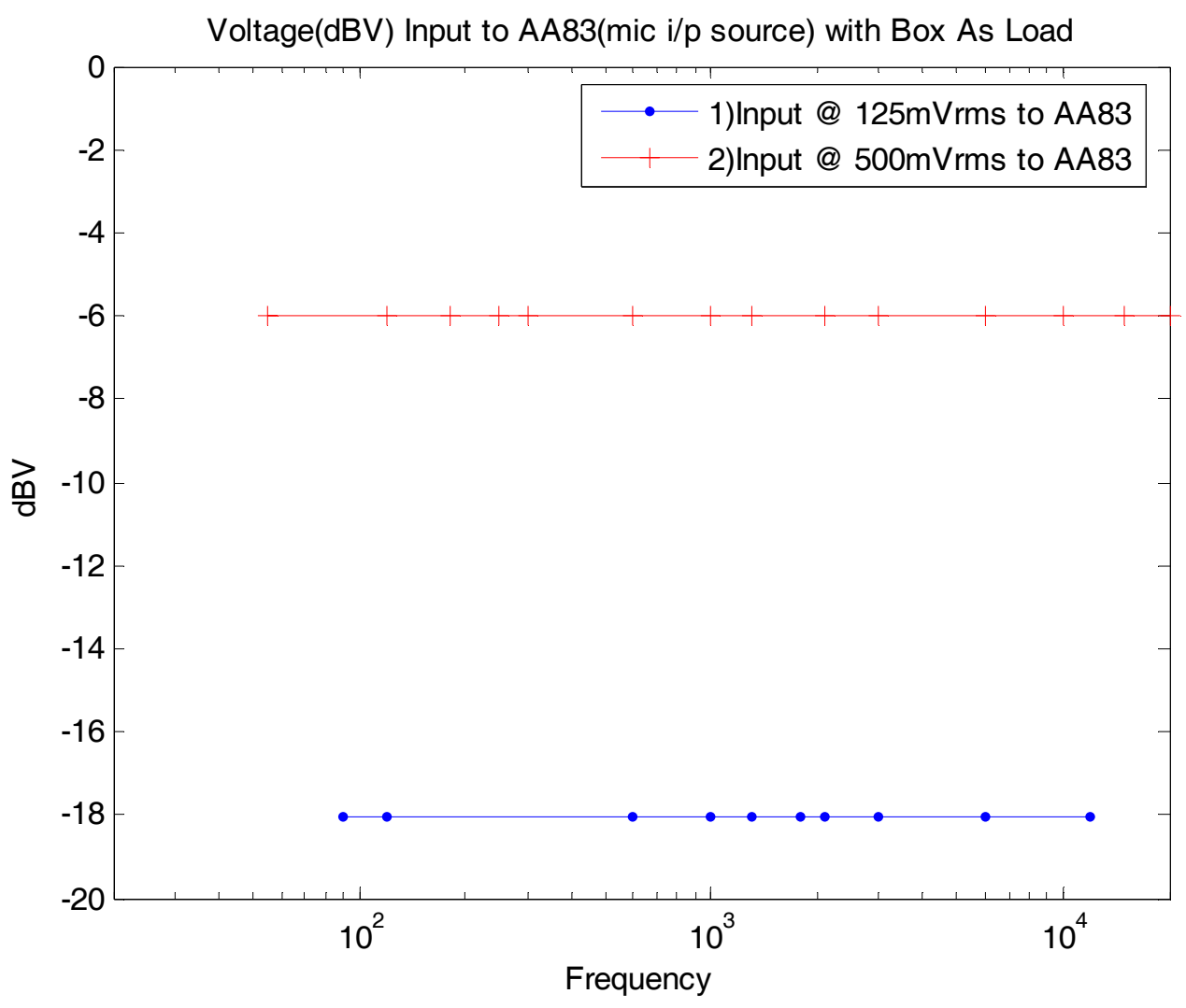

Figure 28 - Voltage input to AA83 (mic i/p) with box 


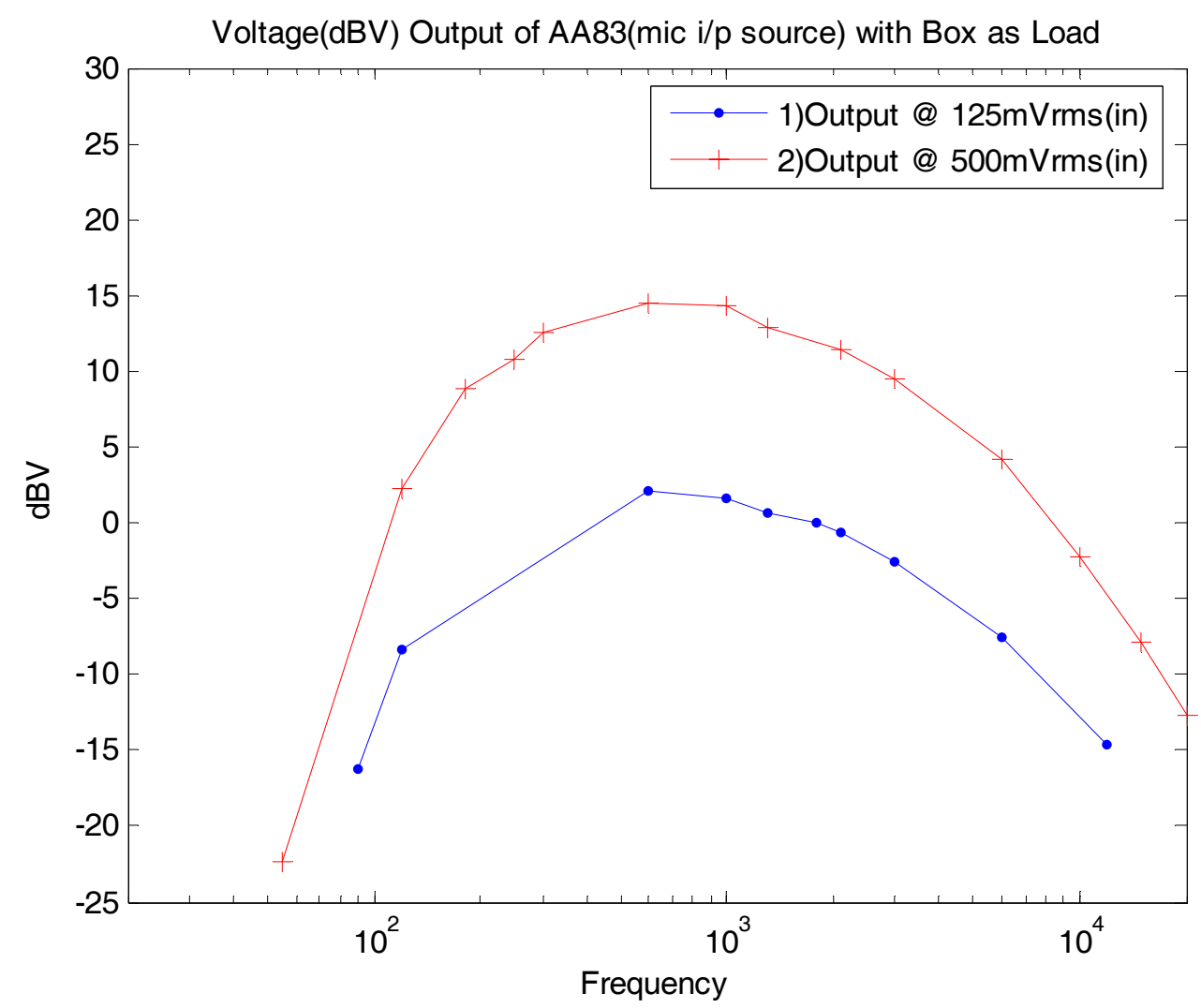

Figure 29 - Frequency Response of AA83 (mic i/p) with Impedance box as load

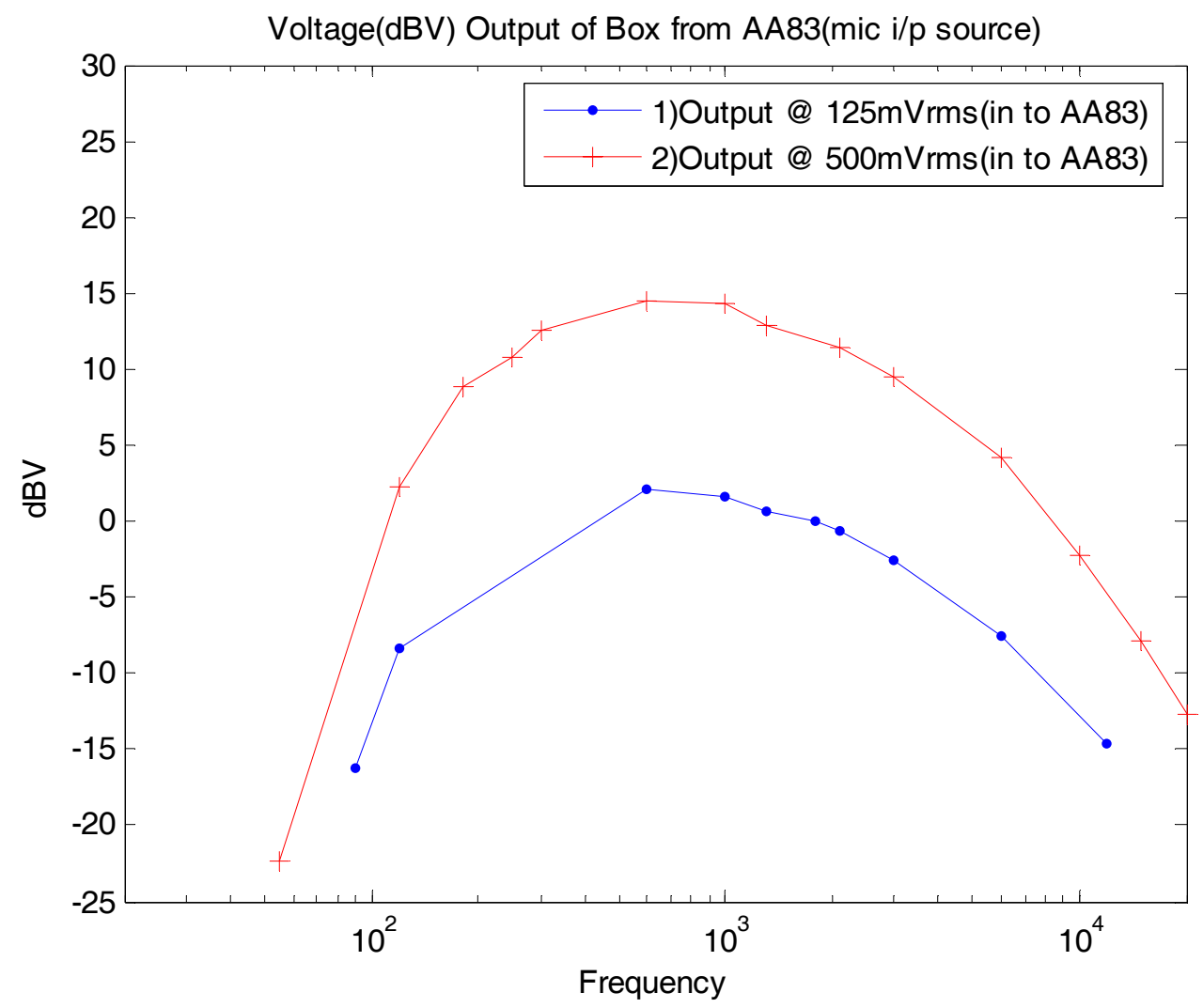

Figure 30 - Frequency Response of Impedance box driven by the AA83(mic i/p) with $150 \mathrm{Ohm}$ headset load 


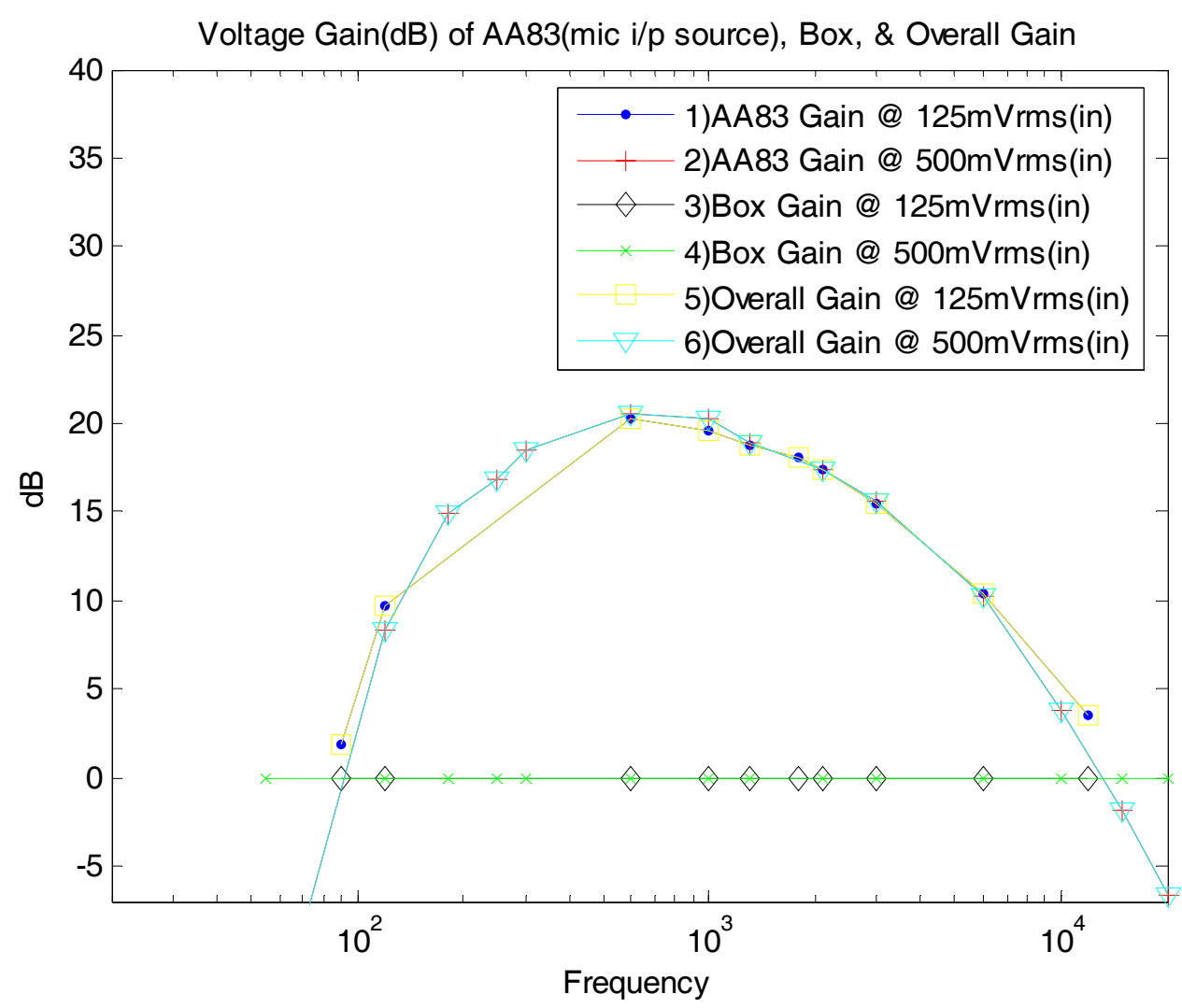

Figure 31 - Calculated Voltage gain of AA83(mic i/p), box, \& overall gain

Apparent Power output from Box[AA83(mic i/p)] to 150 Ohm

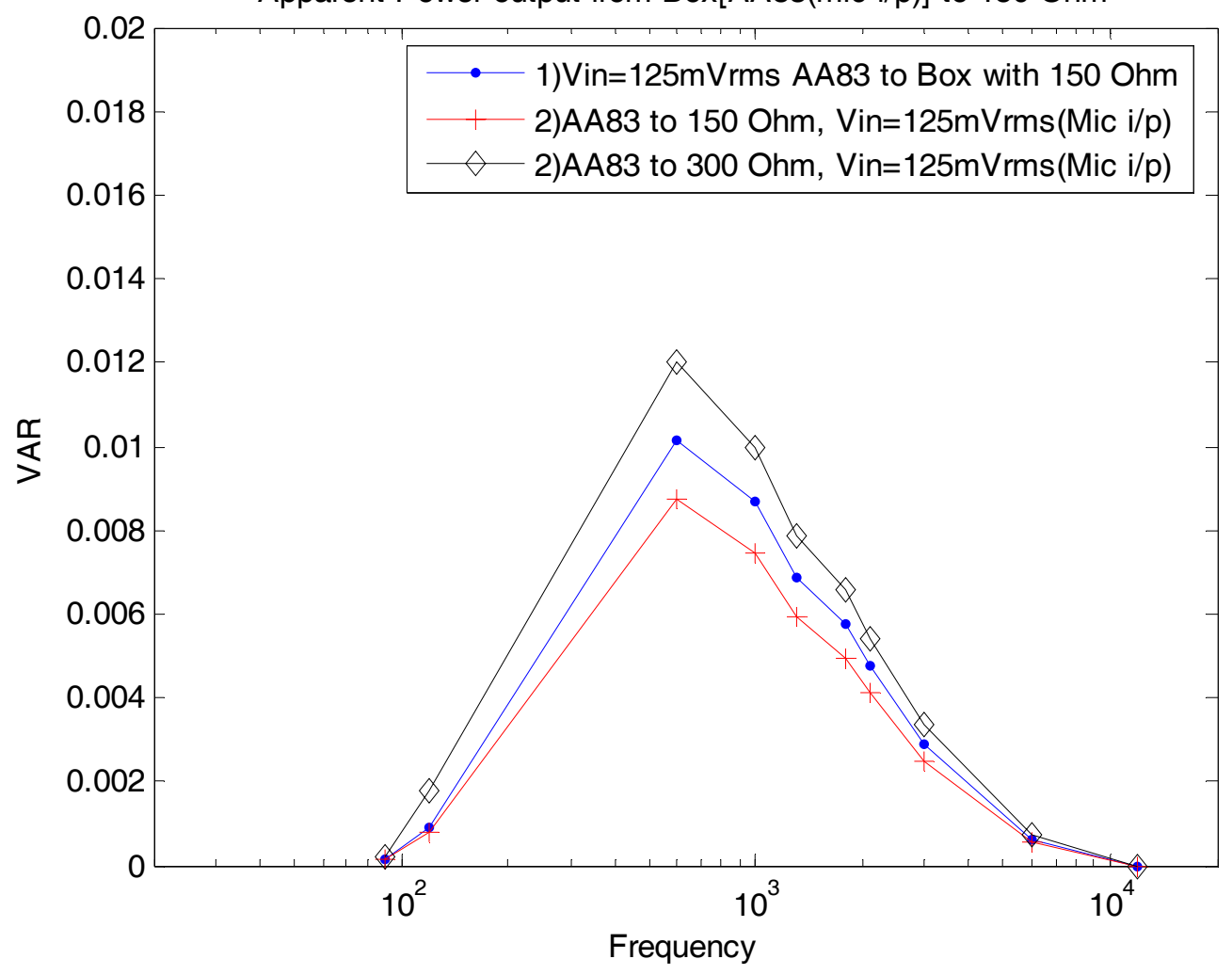

Figure 32 - Calculated Apparent Power to $150 \Omega$ HS from box \& AA83(mic i/p) @ 125mVrms 


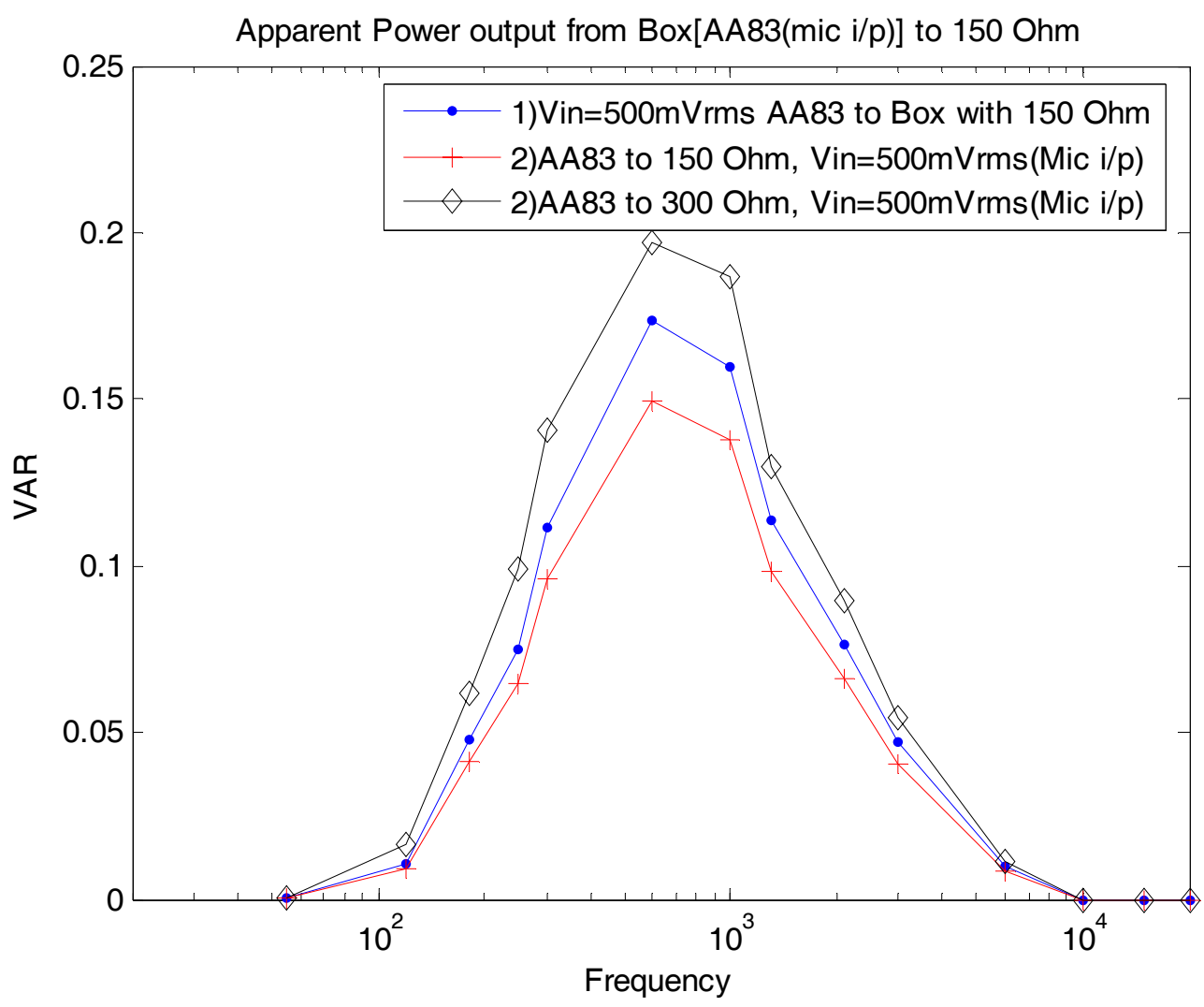

Figure 33 - Calculated Apparent Power to $150 \Omega$ HS from box \& AA83(mic i/p)@ 500mVrms

\subsubsection{Impedance Matching Box Test with Music input to AA83}

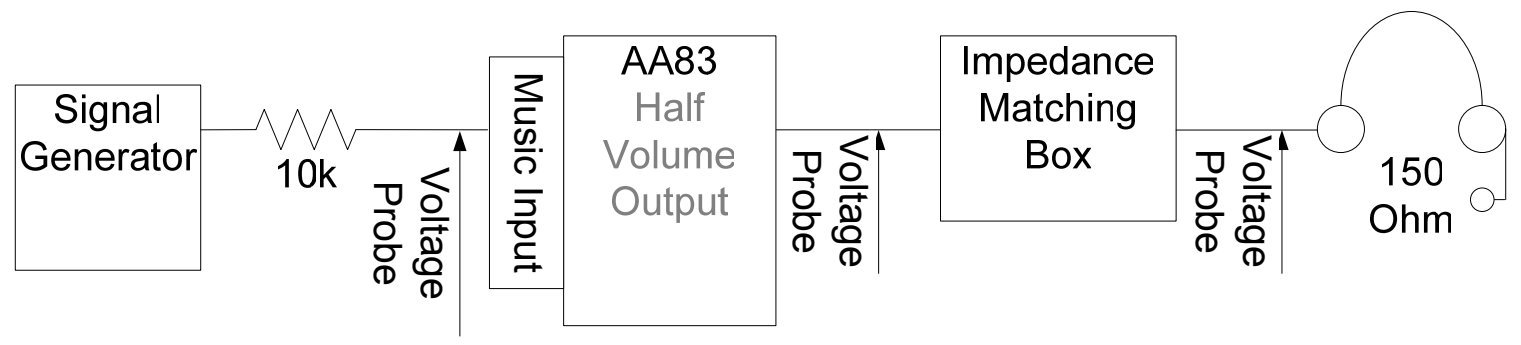

Figure 34 - Impedance box testing setup with AA83 music input 


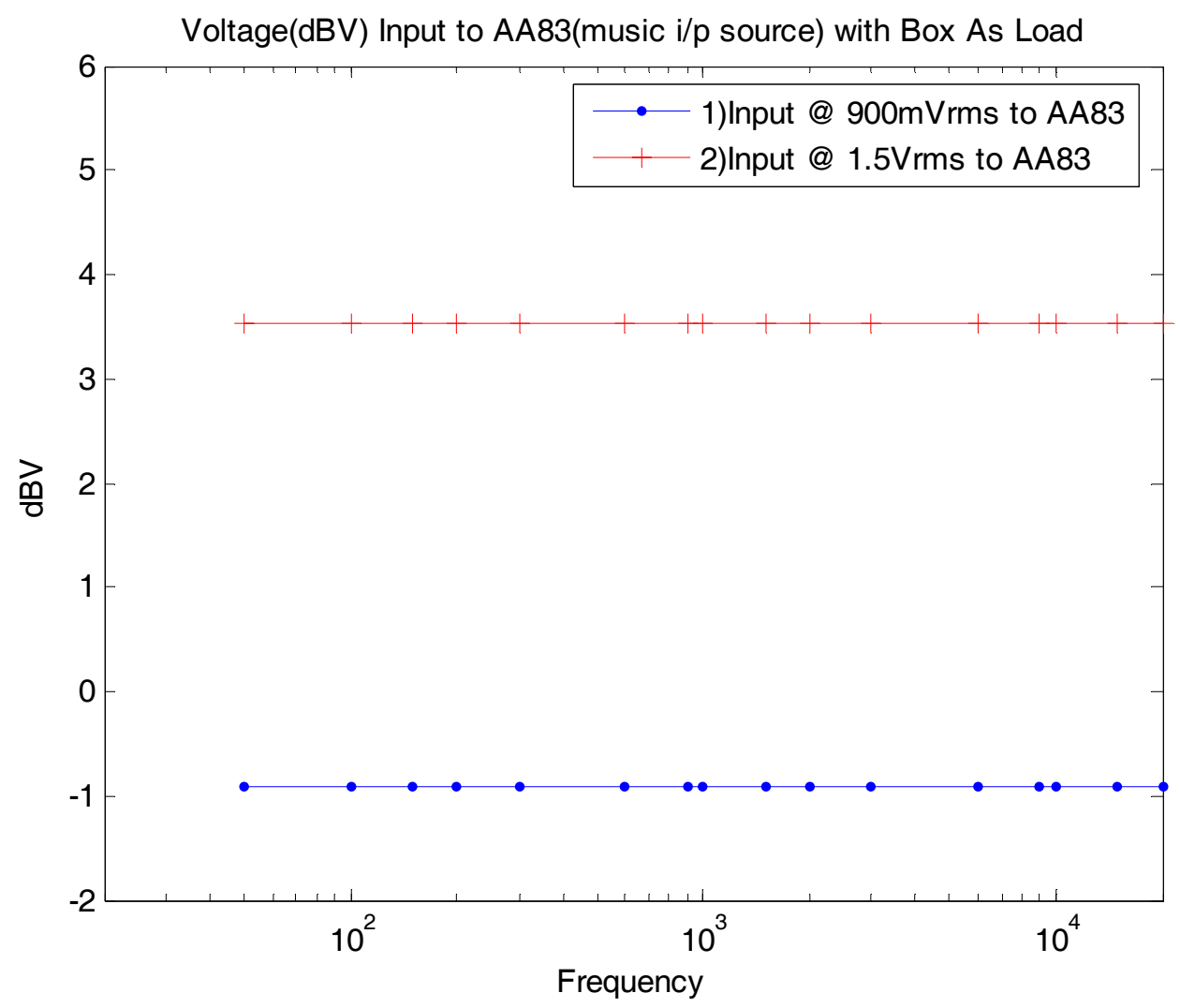

Figure 35 - Voltage input to AA83 (music i/p) with Impedance box as load Voltage(dBV) Output of AA83(music i/p source) with Box as Load

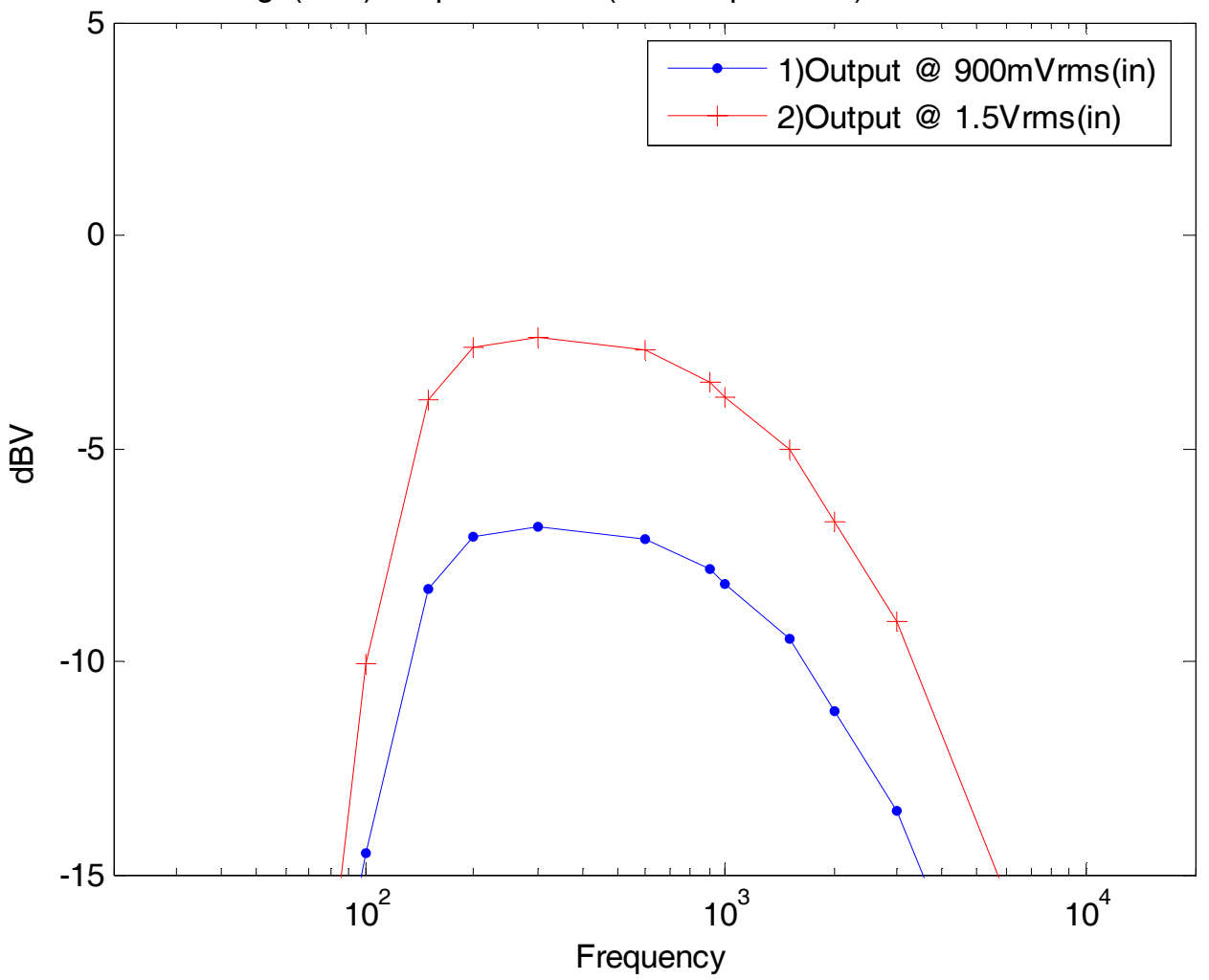

Figure 36 - Frequency Response of AA83 (music i/p) with Impedance box as load 


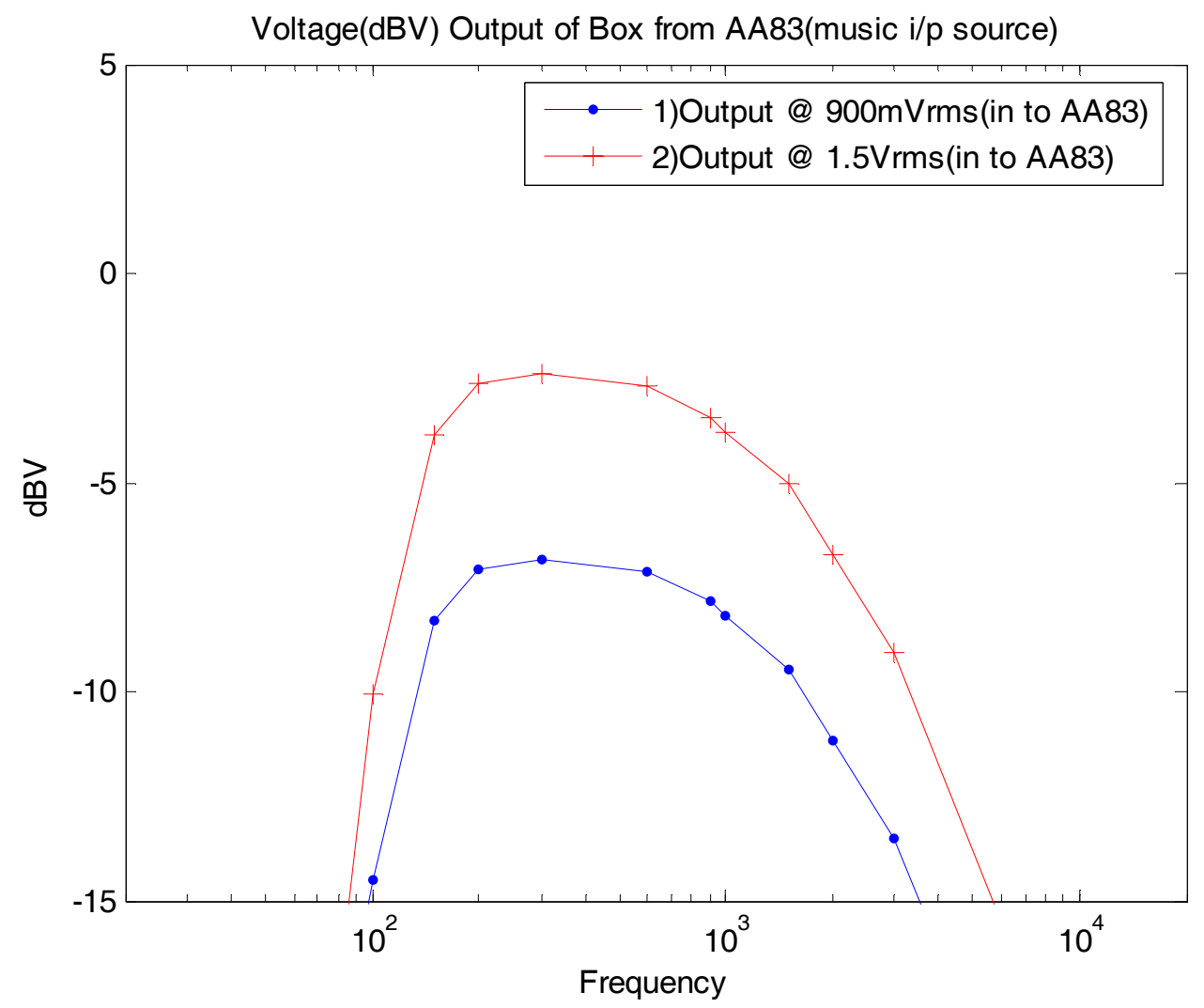

Figure 37 - Frequency Response of Impedance box driven by AA83(music i/p) and $150 \mathrm{Ohm}$ headset load

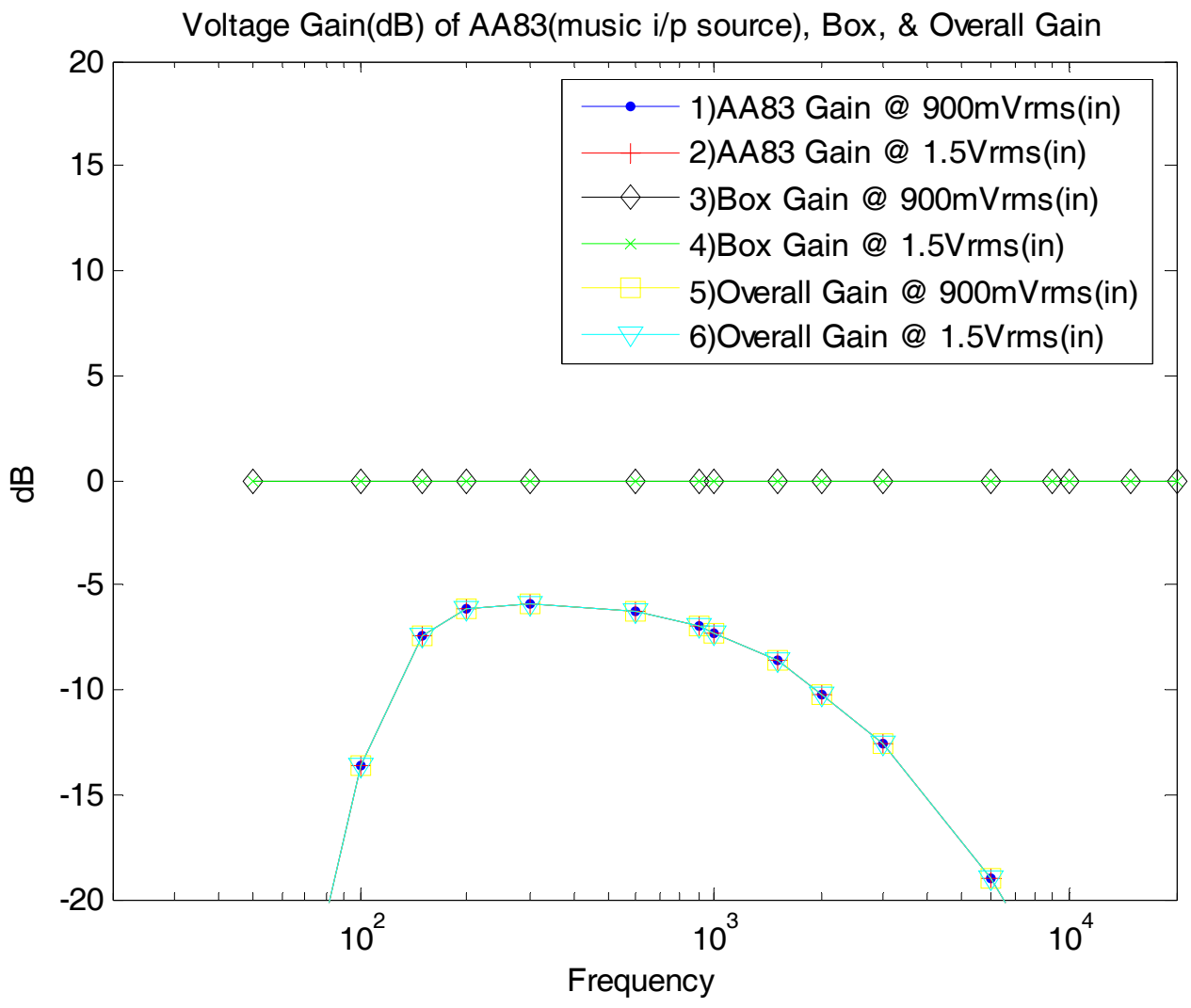

Figure 38 - Calculated Voltage gain of AA83(music i/p), box, \& overall gain 


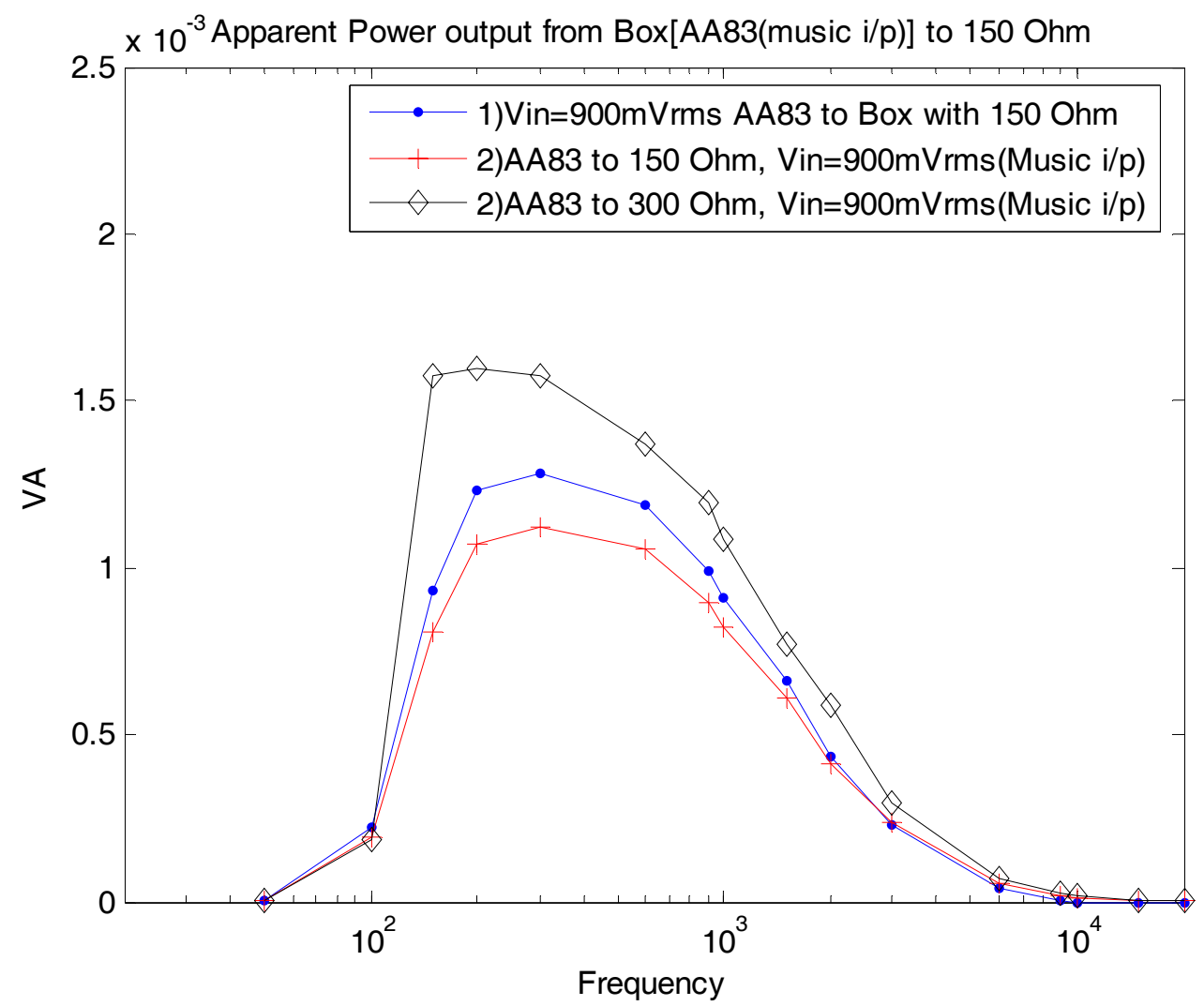

Figure 39 - Calculated Apparent Power to $150 \Omega$ HS from box \& AA83 Music I/P

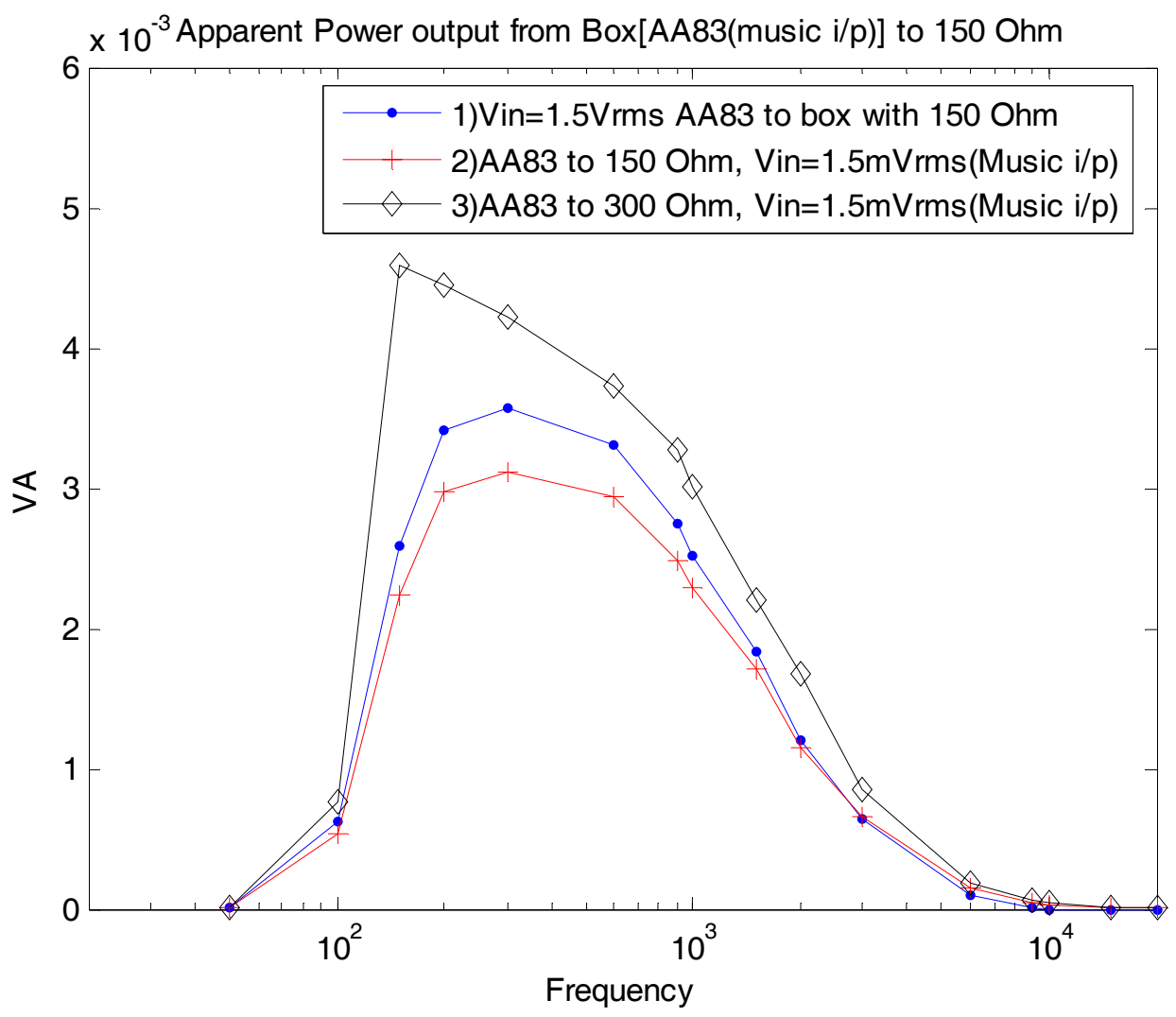

Figure 40 - Calculated Apparent Power to $150 \Omega$ HS from box \& AA83 Music I/P 


\subsubsection{Impedance Matching Box Test without AA83}

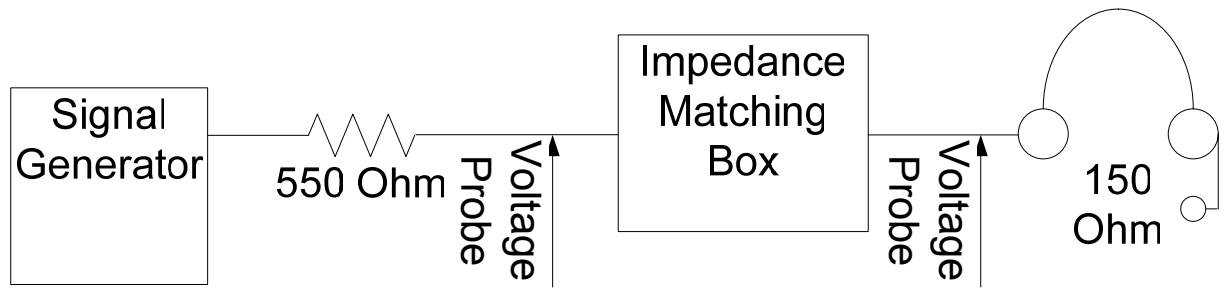

Figure 41 - Impedance Box Individual Testing Setup

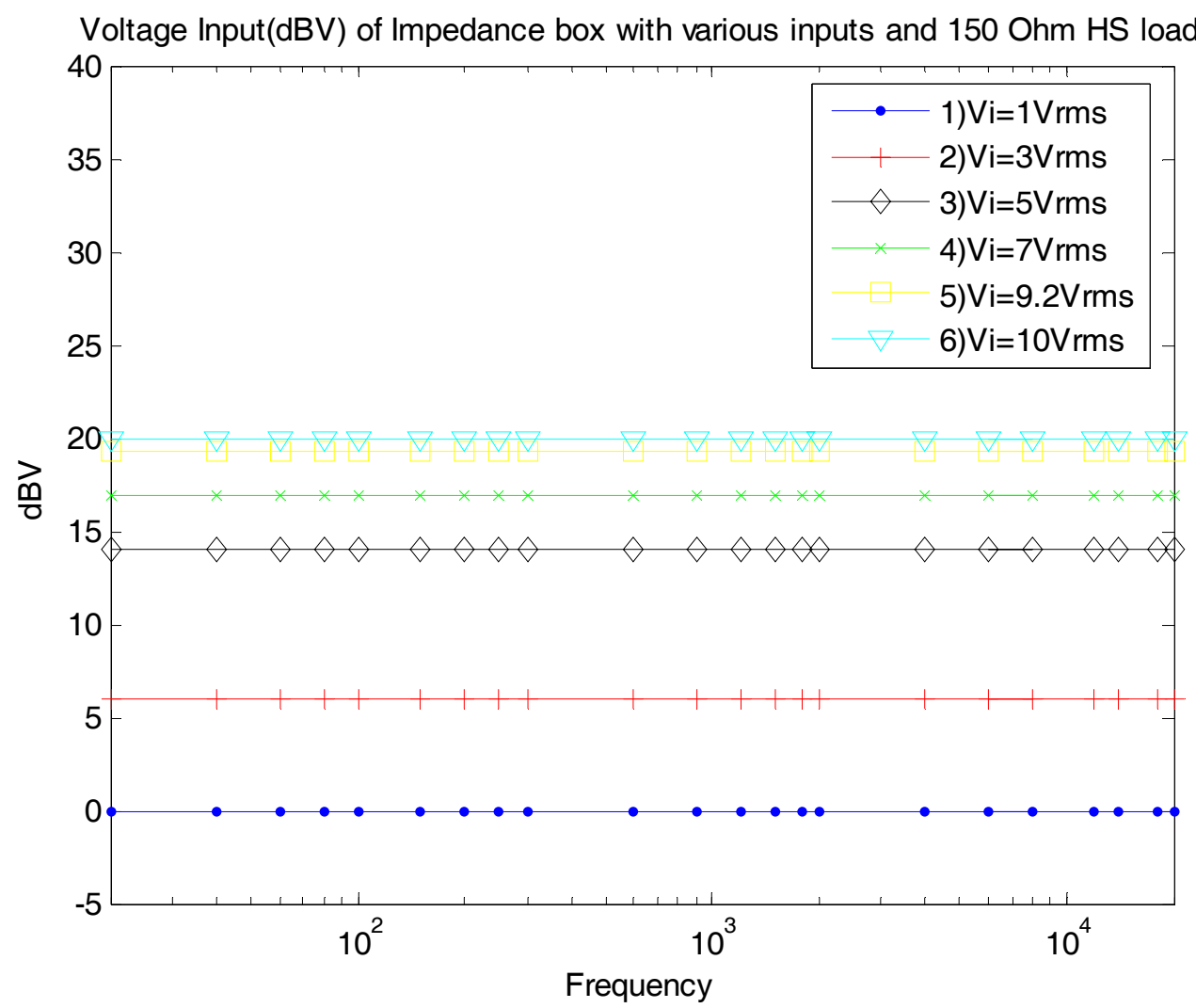

Figure 42 - Voltage input from the Signal Generator to the Box 


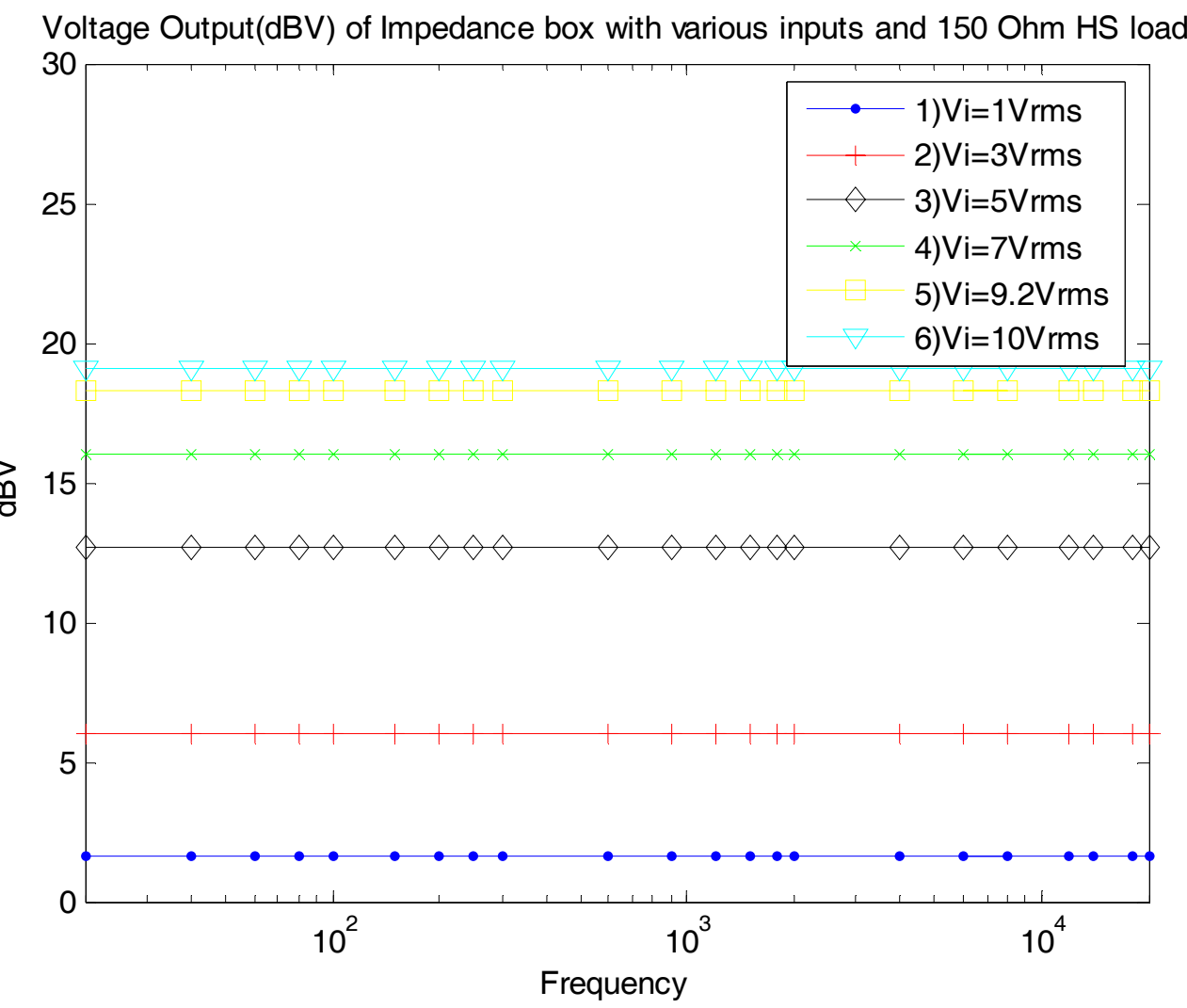

Figure 43 - Frequency Response of the impedance box

Voltage Gain(dB) of Impedance box with various inputs and 150 Ohm HS load

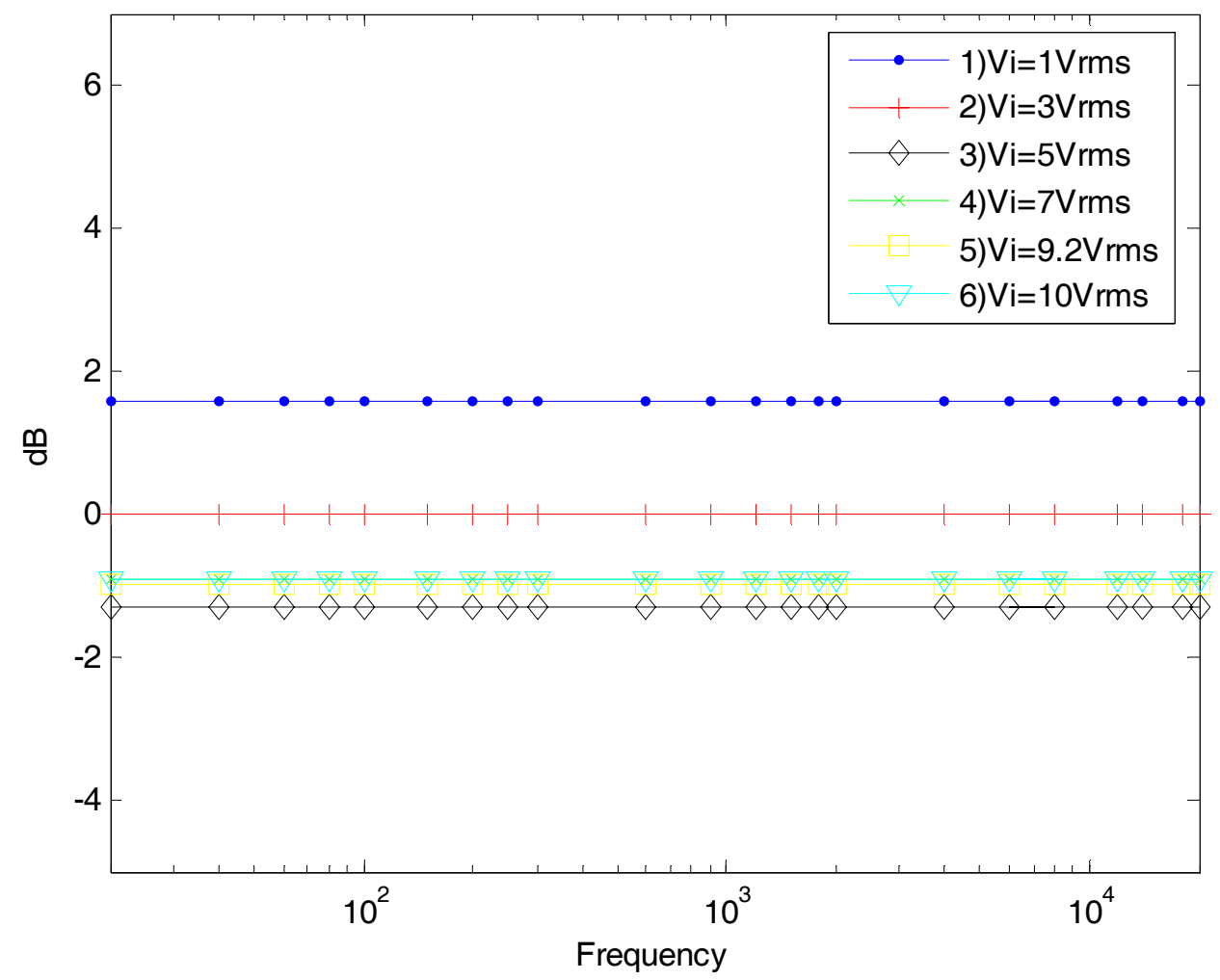

Figure 44 - Frequency Response of the impedance box 


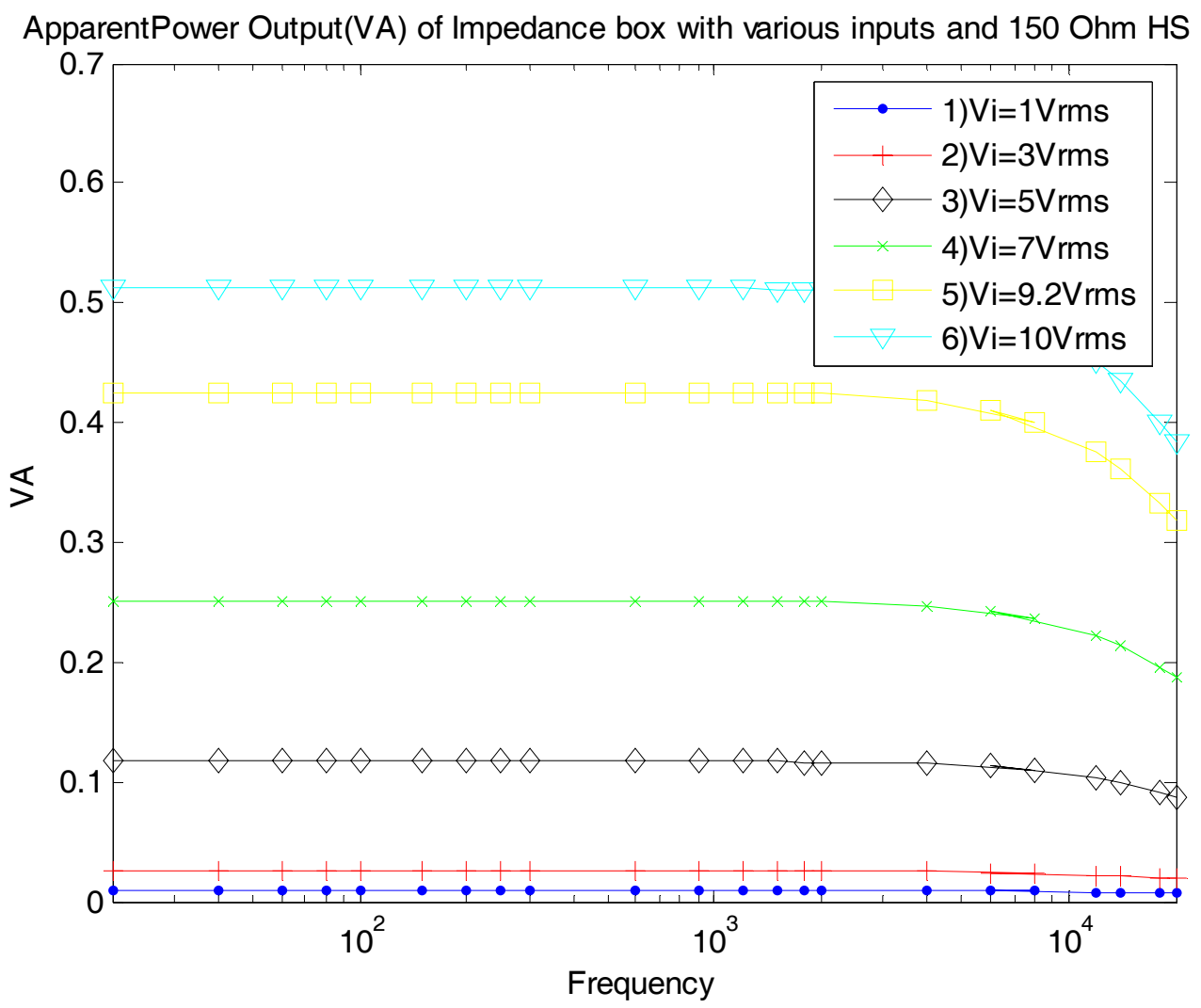

Figure 45 - Calculated Apparent Power to the $150 \Omega$ HS from the box

\subsubsection{Impedance Matching Box Test without power}

This test demonstrates that the impedance matching box is capable of passing signals through the device when power is lost to the device. This is known as a safety pass-through component and was tested with the power turned off only to the impedance matching box, and is shown by Figure 46. Although the power levels delivered to the headset are not the same when directly connected to the AA83, it is very close and the box will let the signal pass when no power is present to the box and is acceptable.

Voltage input to the AA83 is shown by Figure 47. Voltage output from the AA83 and the impedance box without power is shown by Figure 48. Voltage gain of the AA83 and of the AA83 with the impedance box without power is shown by Figure 49. Apparent power delivered to the $150 \mathrm{Ohm}$ headset by the AA83 and through the impedance box without power is shown by Figure 50 . 


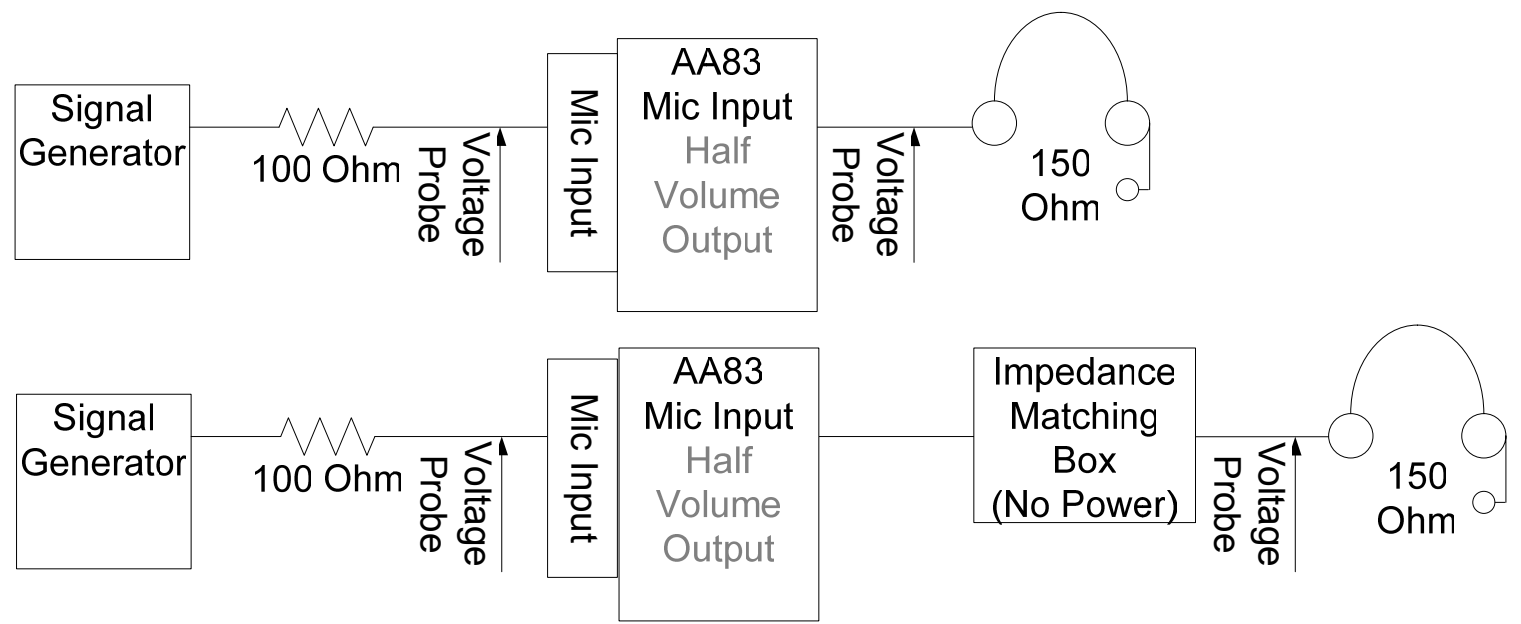

Figure 46 - No Power impedance box testing setup

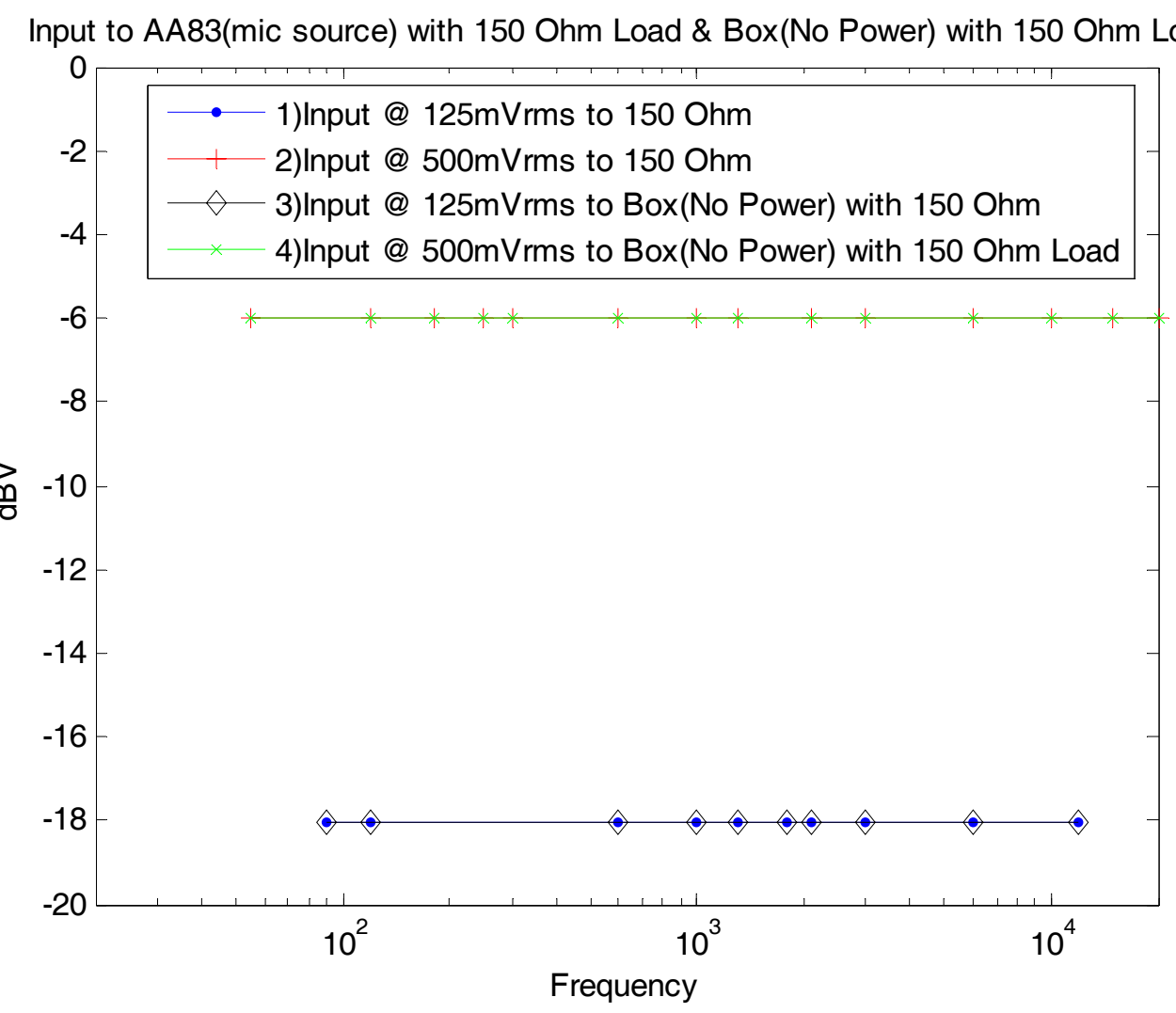

Figure 47 - Input to AA83(mic i/p) \& Box without power 
Voltage(dBV) Output of AA83(mic source) with 150 \& Box(No Power) with 150 Ohm Load

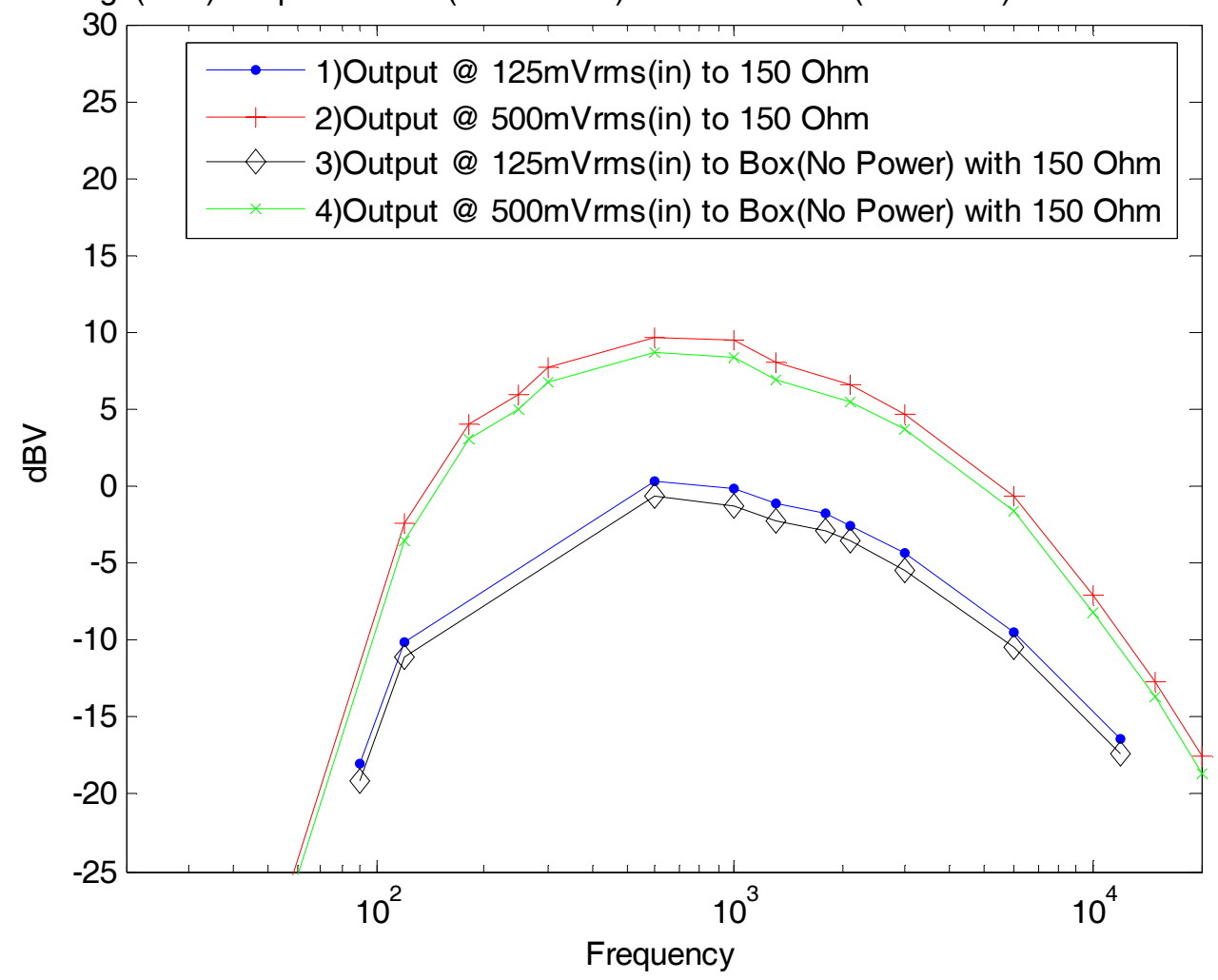

Figure 48 - Frequency Response of AA83(mic i/p) \& Impedance box without power

Voltage Gain(dB) of AA83(mic source) with 150 \& Box(No Power) with 150 Ohm Load

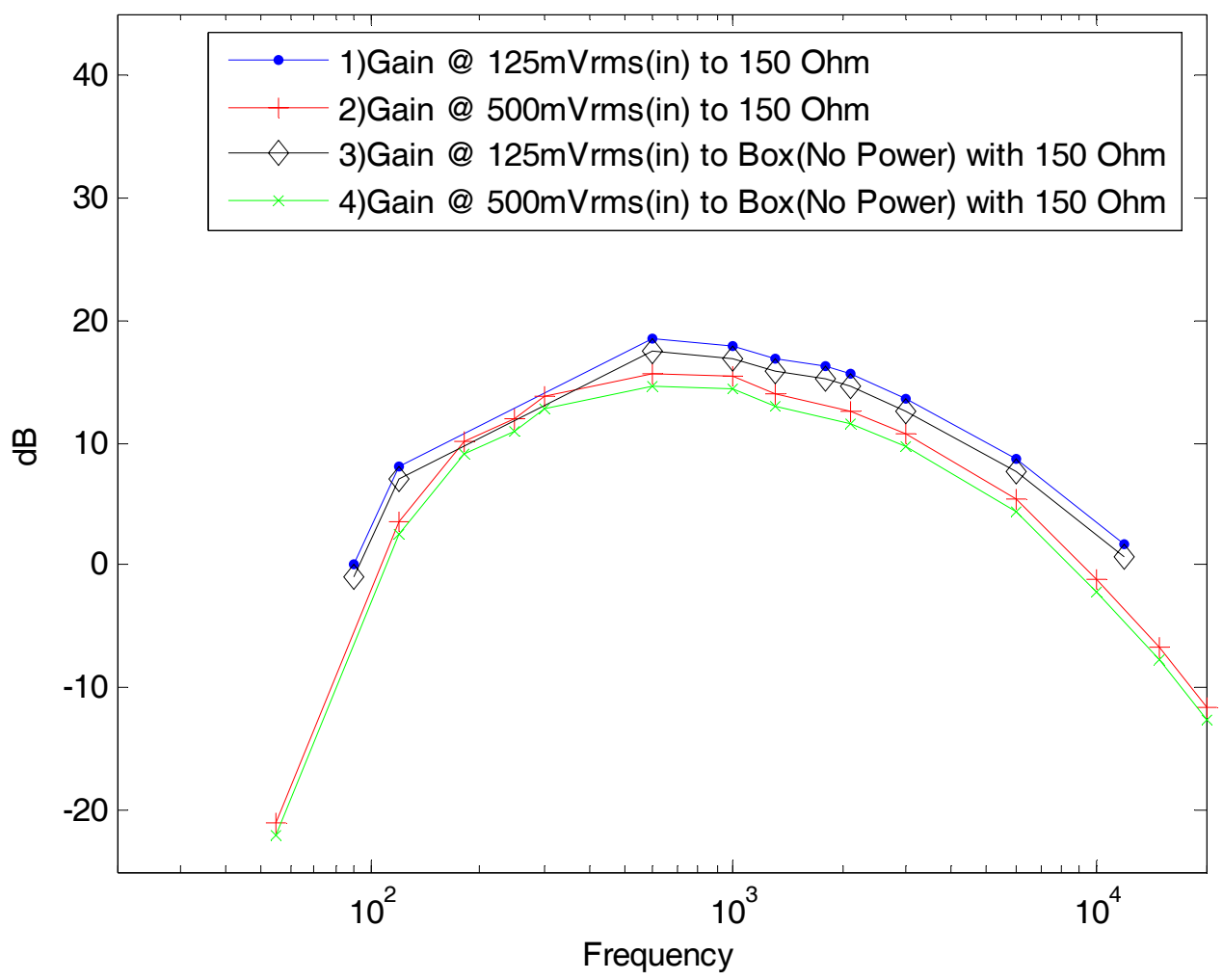

Figure 49 - Calculated Voltage Gain of AA83(mic i/p) \& Box without power 


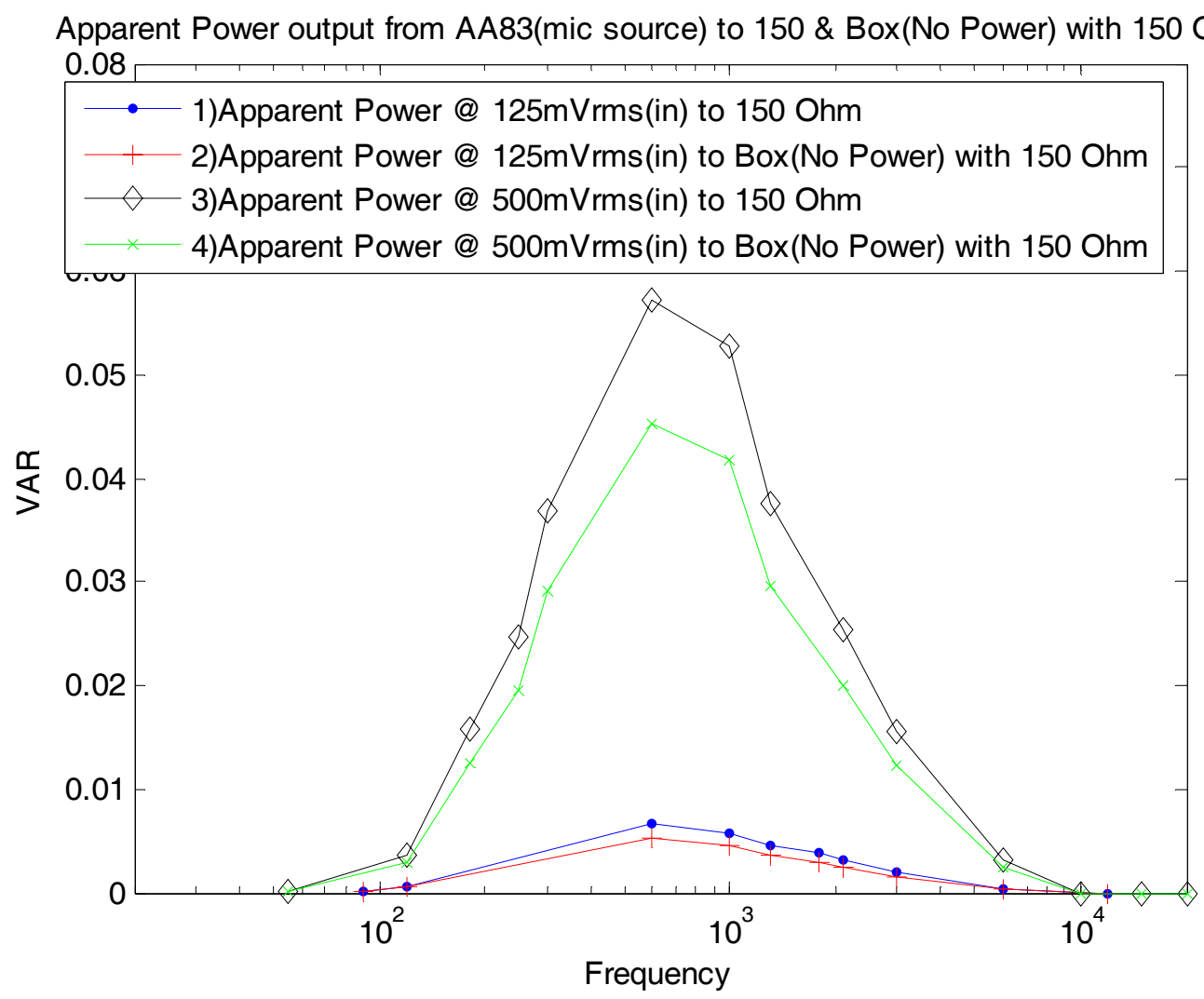

Figure 50 - Calculated Apparent power to $150 \Omega$ HS from AA83(mic i/p) \& Box W/O power

\subsubsection{Impedance Matching Box Tests Conclusion}

The above tests demonstrate properties of the impedance matching box. First,

more power is delivered to the $150 \mathrm{Ohm}$ headset than if the $150 \mathrm{Ohm}$ was directly driven from the AA83, but not as much power as the $300 \mathrm{Ohm}$ headset directly driven from the AA83. Second, the power delivered to the headsets is dominated by the filter inside the AA83. If there was no filter, the power delivered would be flat. Third, if DC supply power is lost to the impedance matching box, there will still be sound delivered to the headsets. The sound level will be much less than if there is DC supply power to the Impedance box but not much less then if the headset were driven directly from the AA83. Overall the impedance matching box seems to perform as designed. This was 1) due to mismatch of impedances to deliver more power to the $150 \mathrm{Ohm}$ headset than the AA83 
would allow and 2) to allow operation when DC supply power is lost to the impedance matching box.

\subsubsection{Sound Level Tests}

Sound Pressure Level (SPL) is another method, besides electrical power, to measure output of a speaker. A SPL meter determines how much air is moved by the speaker and is rated in $\mathrm{dB}$. The sound level tests of the headsets were conducted using a RadioShack digital sound level meter model 33-2055, which reports the sound level in SPL (Sound Pressure Level) dB, which is a measurement of how much sound is pressing on the microphone present in the sound level meter. During this test the frequency weighting was type ' $\mathrm{C}$ ' and the response was slow. The sound level meter was placed very close to the speaker, as shown in Figure 51, and was not moved during testing. The tests conducted have an input frequency range from $20 \mathrm{~Hz}$ to $20 \mathrm{kHz}$, and are shown in Figure 52. The results demonstrate that with the impedance matching box the $150 \mathrm{Ohm}$ level will sound just as loud as the $300 \mathrm{Ohm}$ headset connected directly to the AA83. Also shown is how low the sound levels of the $150 \mathrm{Ohm}$ headset are when connected directly to the AA83 and demonstrating the need for the impedance matching box. The volume level remained in the middle position during testing and the microphone input on the AA83 was used as the signal input. 
1) $300 \mathrm{Ohm}$ to $\mathrm{AA} 83$

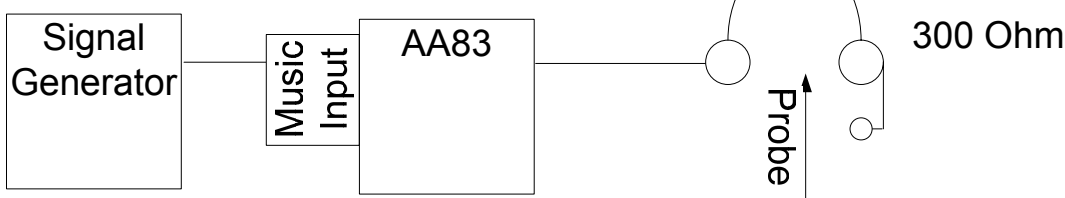

2) $150 \mathrm{Ohm}$ to $\mathrm{AA} 83$

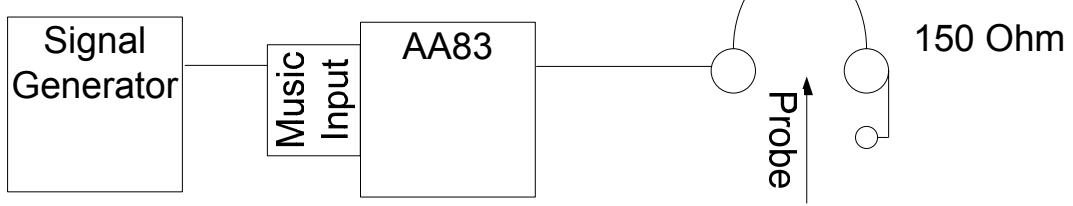

3) $150 \mathrm{Ohm}$ to Impedance Box (Powered)

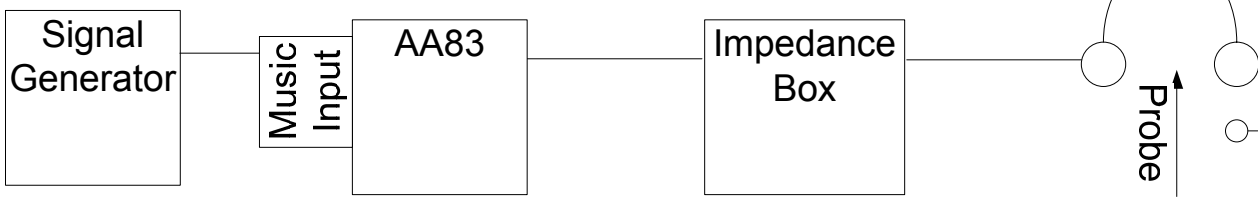

$150 \mathrm{Ohm}$

4) $150 \mathrm{Ohm}$ to Impedance Box (No Power)
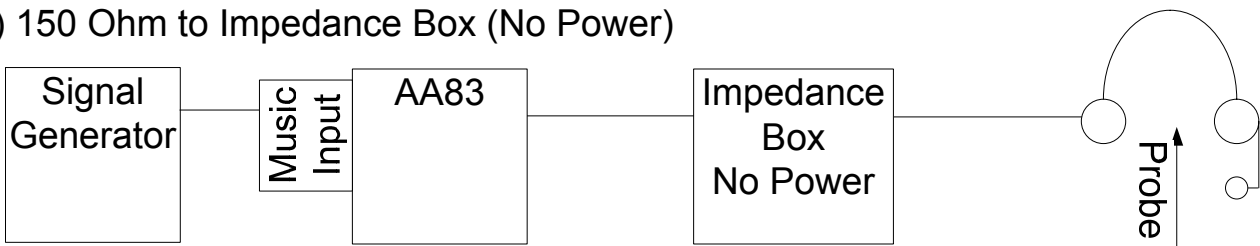

$150 \mathrm{Ohm}$

Figure 51 - Sound Level Test Setup

Measured Audio Frequency Response

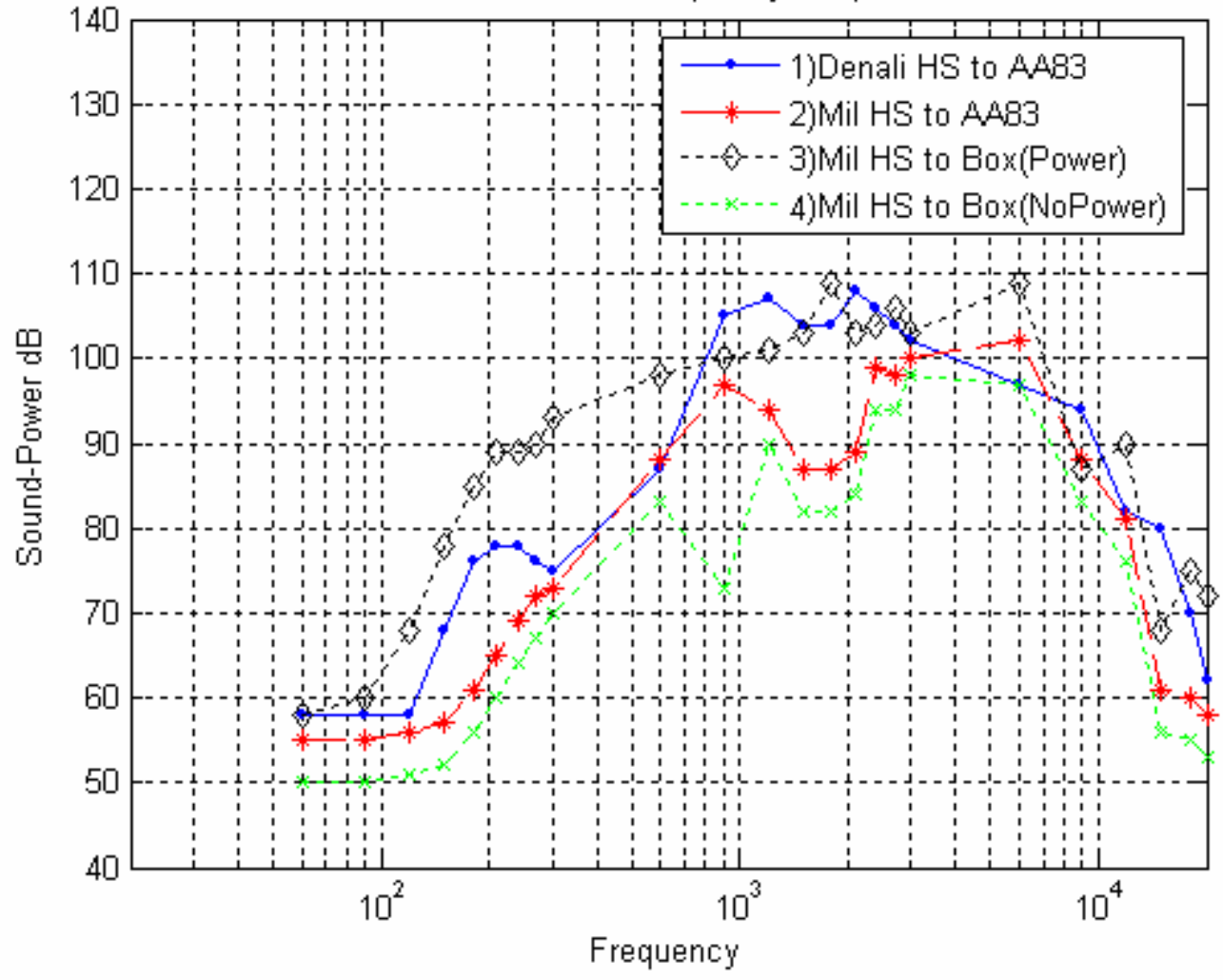

Figure 52 - Sound level test graph 


\subsubsection{Dynamic Microphone Amplifier Circuit Tests}

The dynamic microphone amplifier test consists of frequency responses $(300 \mathrm{~Hz}$ to $3 \mathrm{kHz}$, which estimates what a human voice can produce) in various points in the circuit with and without a load. The signal generator did not output a low enough voltage for the circuit, so an external circuit was required to drop the voltage level to acceptable levels $(\sim 20 \mathrm{mV})$. This circuit design of the voltage reducer, shown in Figure 53, needed capacitors to filter the high frequency components that are not wanted and disrupted testing during this measurement.

The voltage gain input frequency response of the microphone circuit test was conducted to demonstrate the flat signal coming from the voltage reducer preformed as it was designed. The test setup can be shown in Figure 54. The test setup involved the open loop, $1 \mathrm{k}$ Ohm resistive load, and the NAT AA83 microphone input response of the impedance matching box with the dynamic microphone component.

The voltage of the signal generator is shown by Figure 55 . The output voltage of the reduction circuit is shown by Figure 56. The inputs and outputs of the impedance matching box in this test were the dynamic microphone input and the microphone output. The output of the impedance box is shown by Figure 57 . The voltage gain of the reducer, shown by Figure 58, was calculated by the input to the reducer and the output of the reducer. The voltage gain of the dynamic microphone circuitry of the impedance matching box, shown by Figure 59, was calculated with the input to the box and its output. The overall gain of the entire circuit is shown by Figure 60, and was calculated from the signal generator input and the impedance box output. 


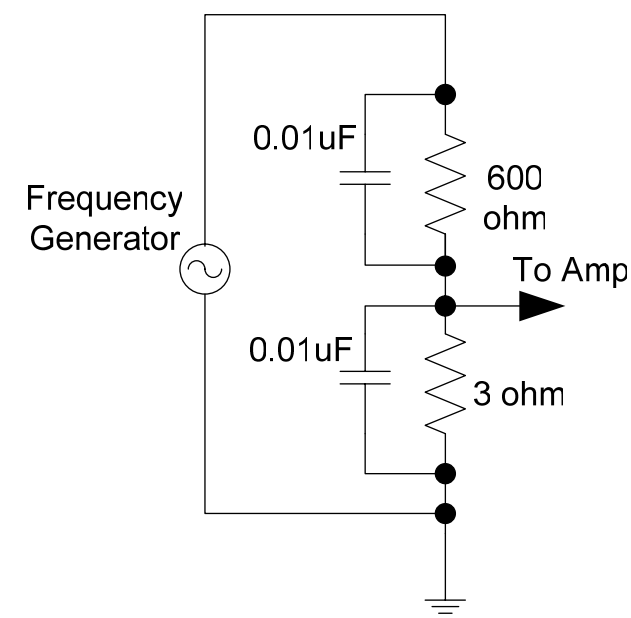

Figure 53 - Voltage Reduction Circuit

1) Output No Load

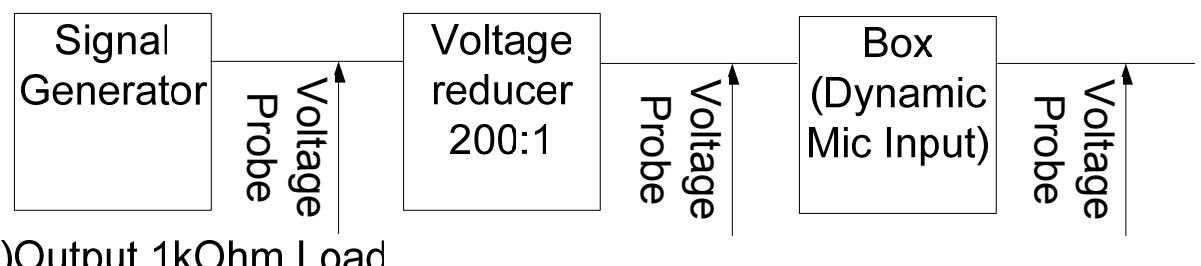

2)Output $1 \mathrm{kOhm}$ Load

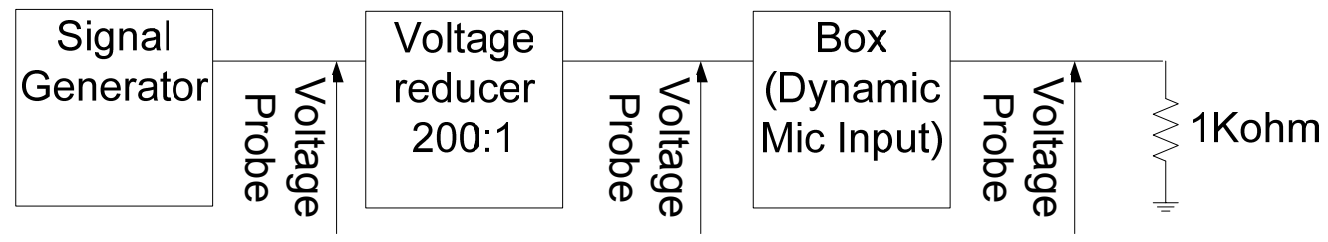

3) Output to AA83 Open

\begin{tabular}{|c|c|c|c|c|c|c|}
\hline \multirow{2}{*}{$\begin{array}{c}\text { Signal } \\
\text { Generator }\end{array}$} & & \multirow{2}{*}{$\begin{array}{c}\text { Voltage } \\
\text { reducer } \\
200: 1\end{array}$} & & \multirow{2}{*}{\begin{tabular}{|c|} 
Box \\
(Dynamic \\
Mic Input)
\end{tabular}} & & \multirow{2}{*}{$\begin{array}{c}\text { AA83 } \\
\text { Mic Input }\end{array}$} \\
\hline & 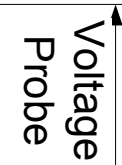 & & 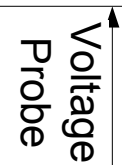 & & 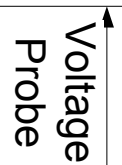 & \\
\hline
\end{tabular}

Figure 54 - Microphone Circuit Input Response Setup 


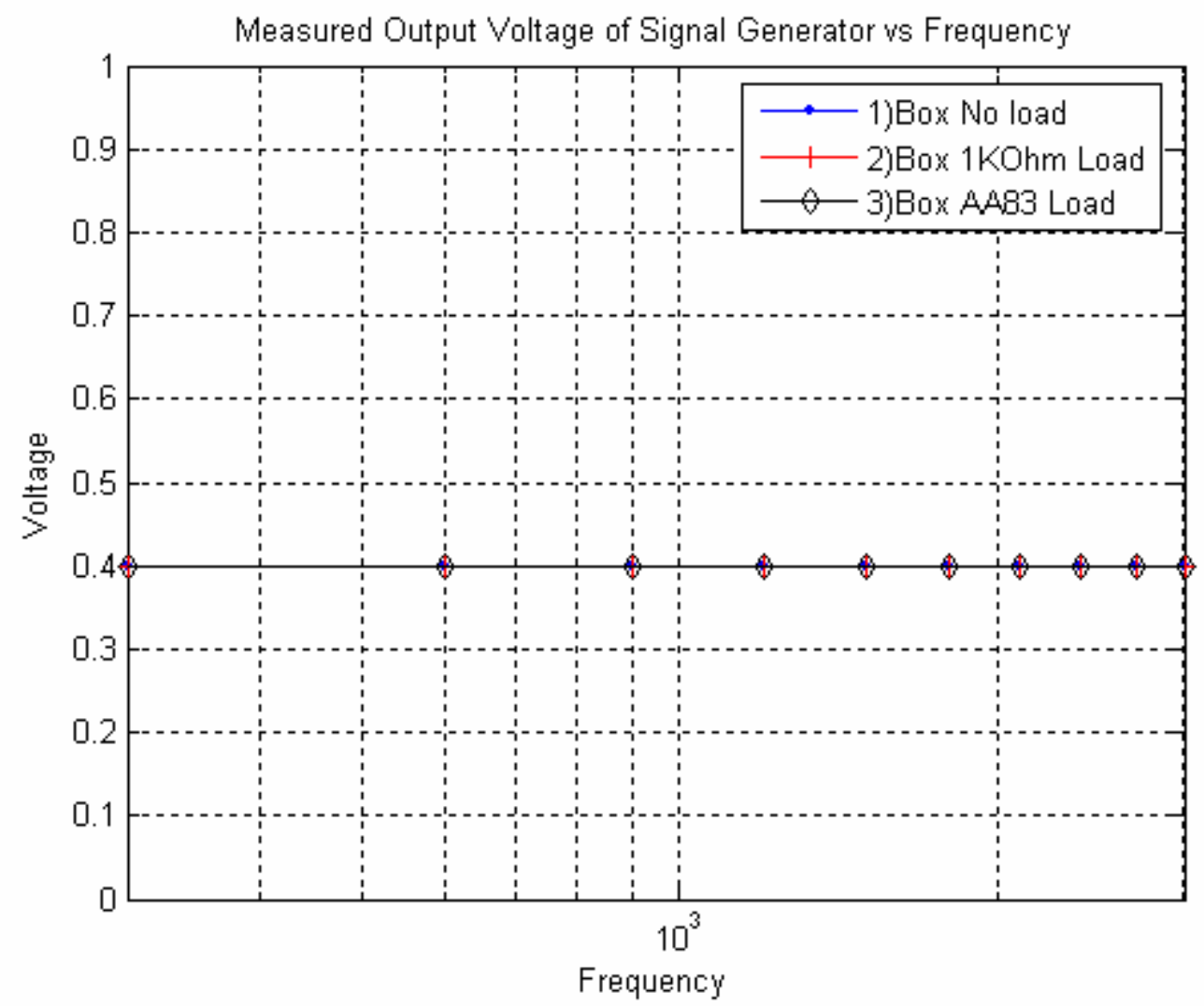

Figure 55 - Microphone Test Signal Generator Output

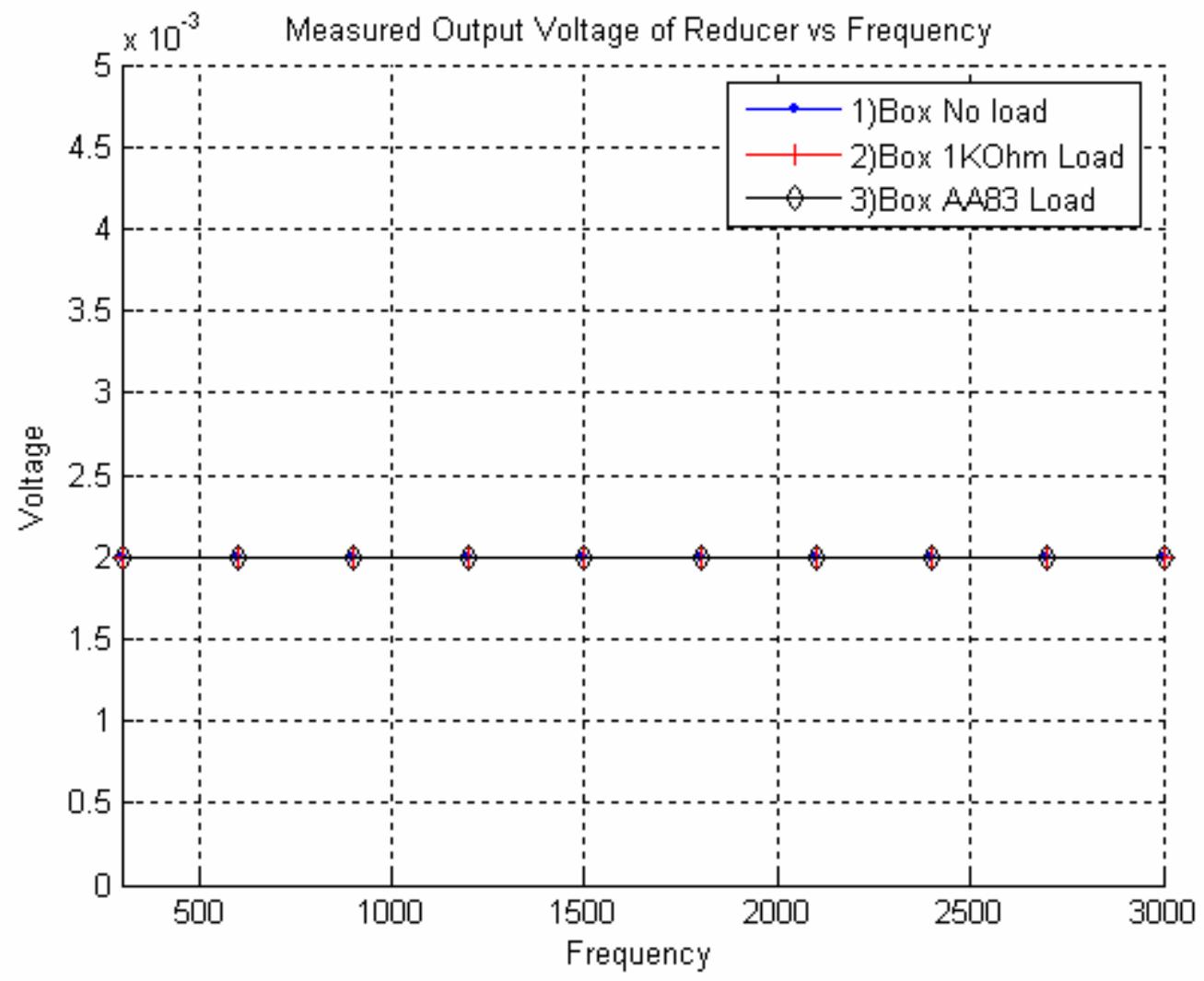

Figure 56 - Microphone Test Reducer Output 


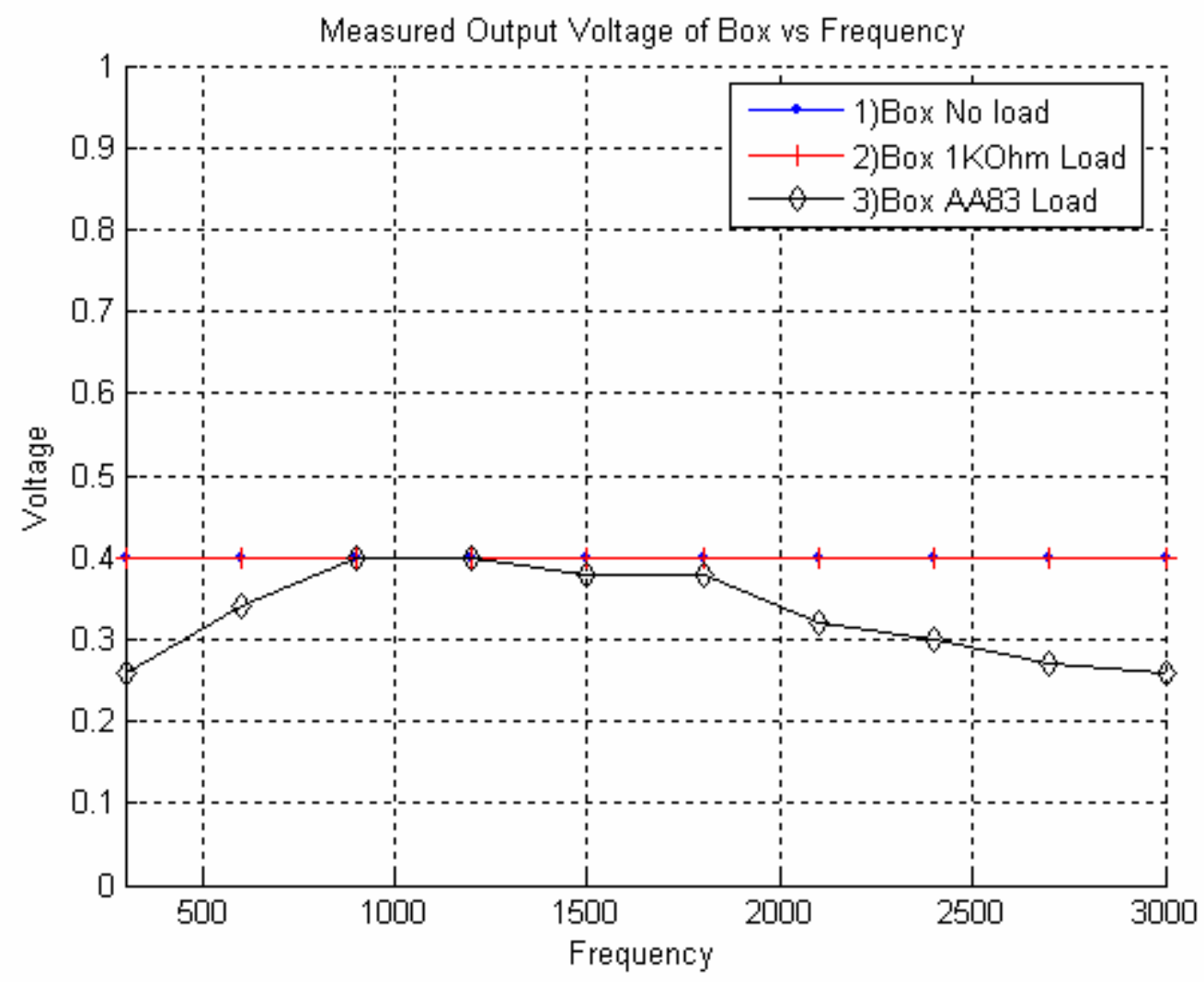

Figure 57 - Microphone Test Impedance Box Output

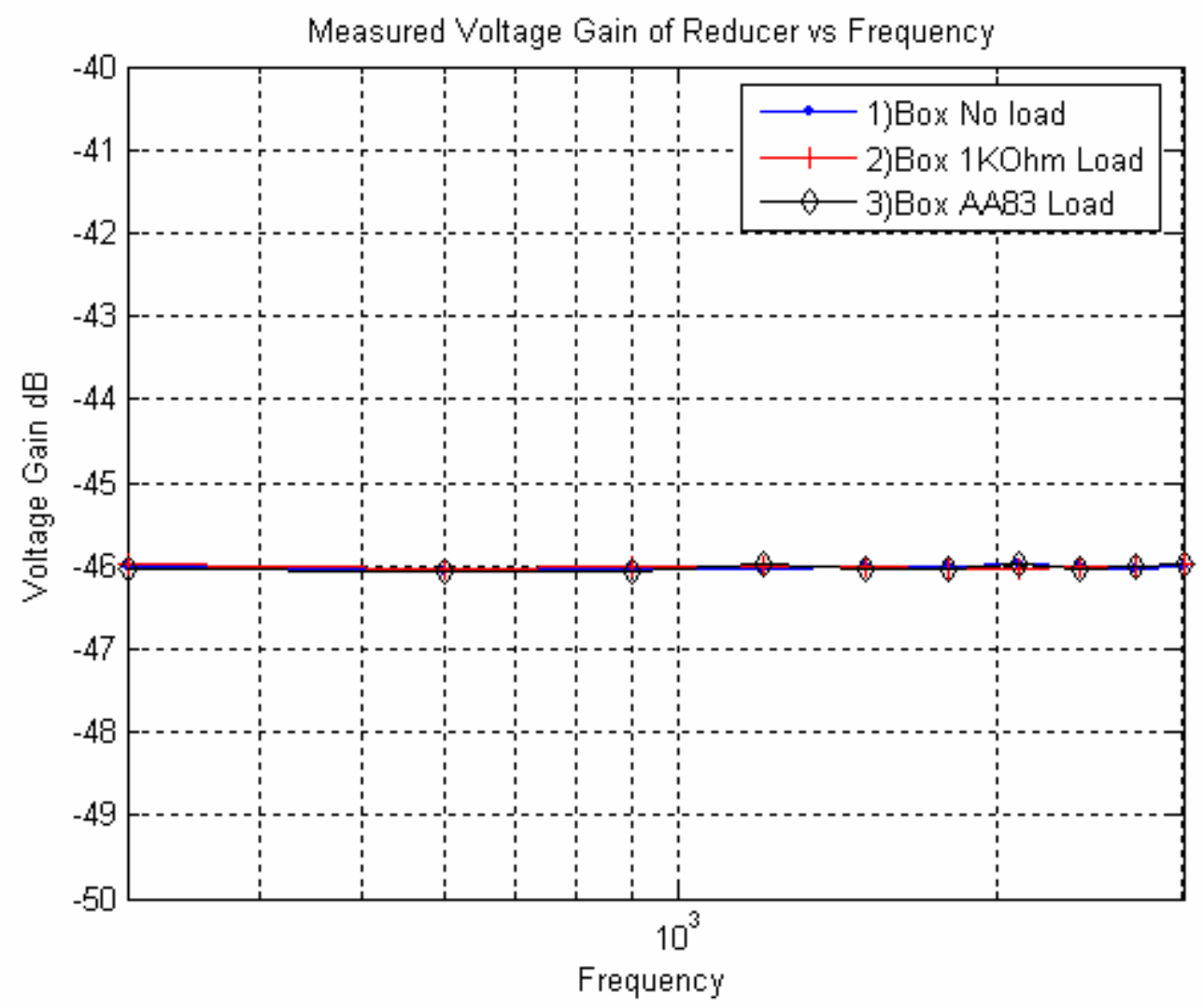

Figure 58 - Calculated Voltage Gain of Reducer 


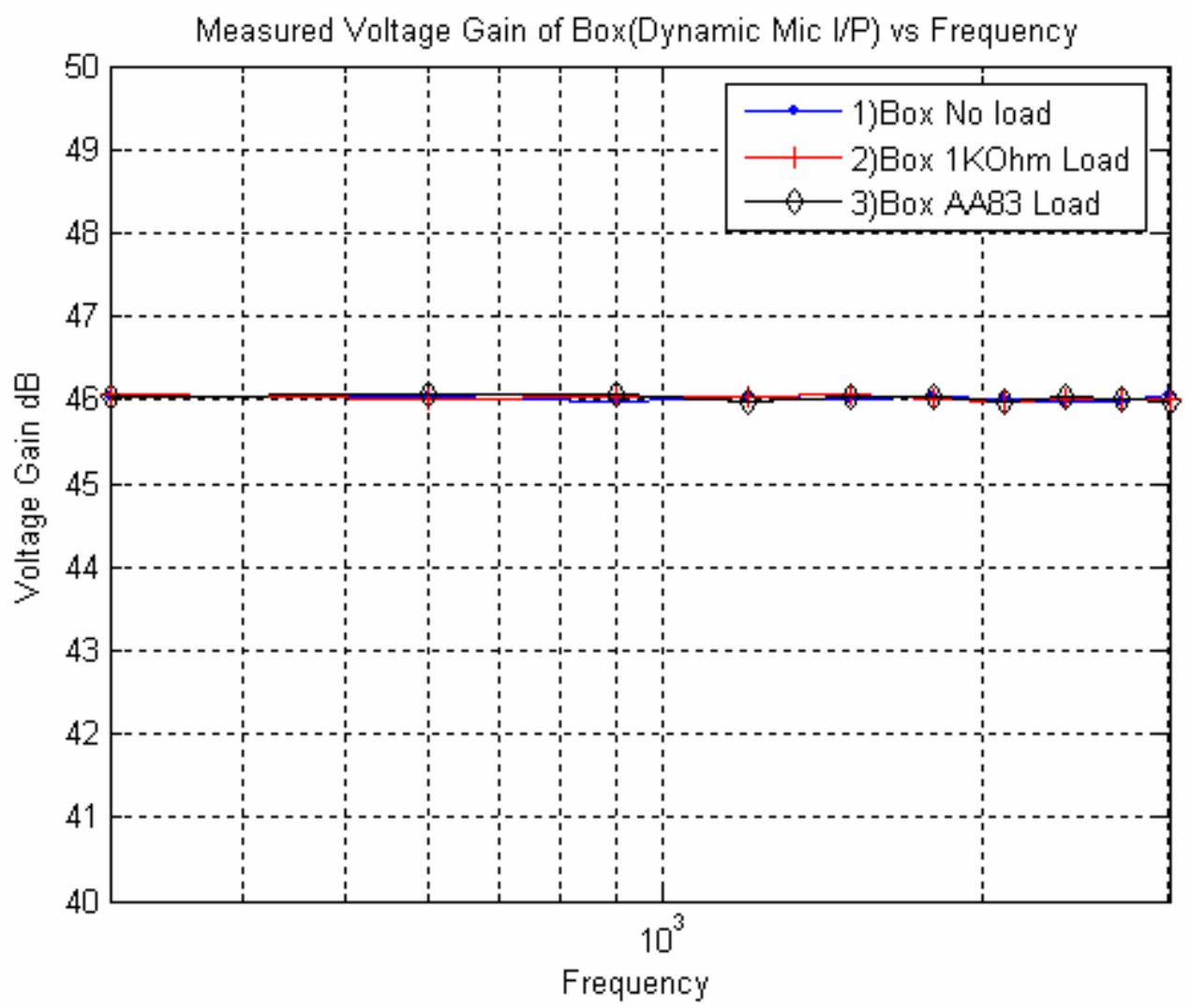

Figure 59 - Calculated Voltage Gain of Box

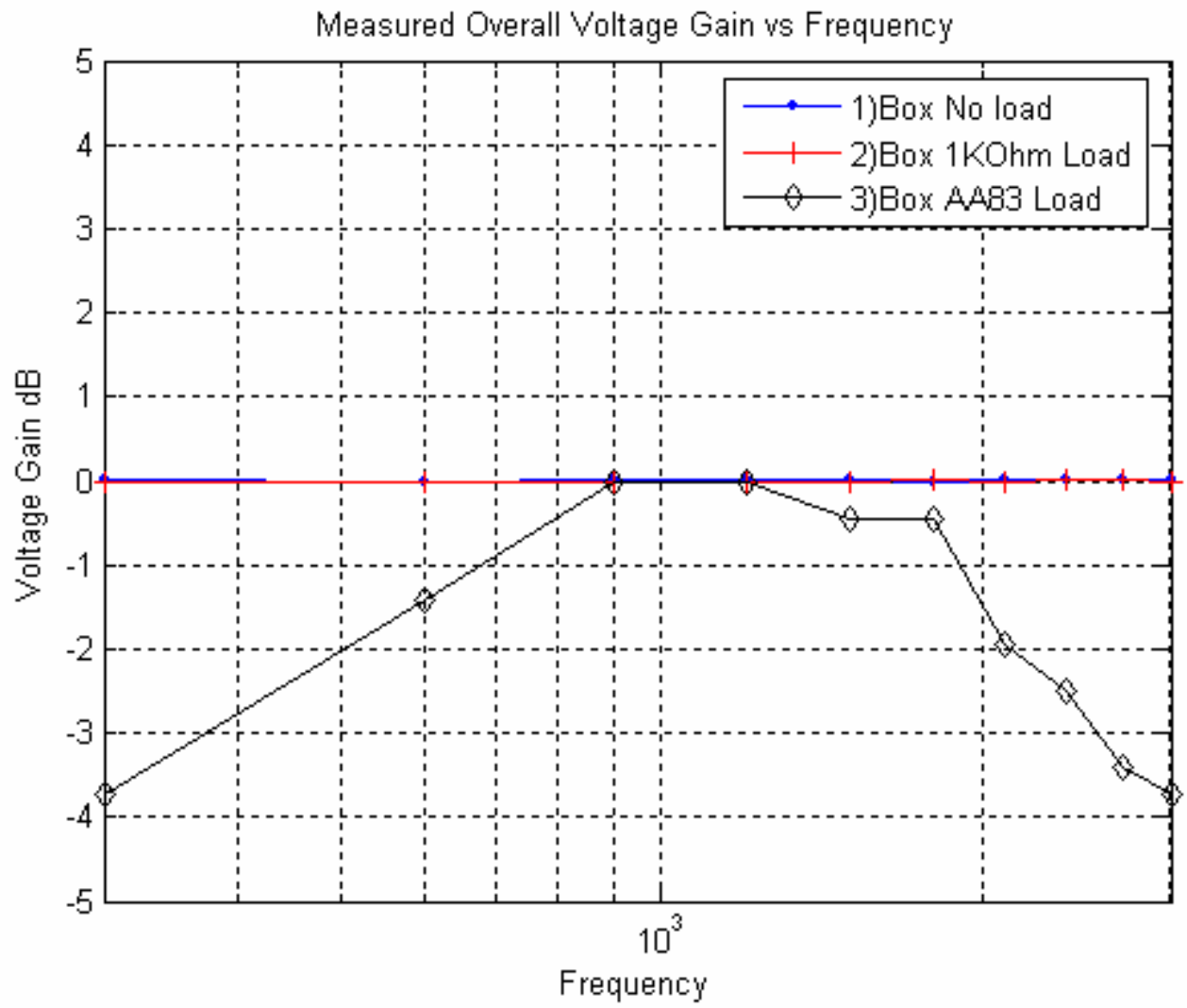

Figure 60 - Calcualted Overall Voltage Gain of Reducer \& Box 


\subsubsection{Dynamic Microphone Amplifier Circuit Tests Conclusion}

The above test demonstrates that the dynamic microphone amplifier in the impedance matching box perform as designed. The goal of the design was to have a constant voltage gain of 200 for the input. The final test where the NAT AA83 received input from the impedance matching box demonstrates that the AA83 contains a filter that is centered around the middle of the human voice range, and does not affect the performance of the impedance matching box.

\subsubsection{Overall Tests Conclusion}

The measurements taken demonstrate two dominate properties. First, the AA83 clearly has a filter that is most likely used to remove unwanted frequencies from being attenuated through the device. The good part about this filter is that unwanted noise will not be propagating through the device creating a cleaner sound. The bad part is that very high and very low frequencies are removed or greatly hindered, which will reduce sound quality from music sources. Second, the impedance matching device and its underlying circuitry perform as it was designed. The impedance matching device boosted the power, which in turn boosted the sound output to let $150 \mathrm{Ohm}$ appear almost as loud as $300 \mathrm{Ohm}$ headsets.

The microphone amplification circuit demonstrated a voltage gain of $46 \mathrm{~dB}$ or 200, and the headset circuit brings the power output of the $150 \mathrm{Ohm}$ headset into range similar to that of the $300 \mathrm{Ohm}$ headset. Overall the use of a transformer would probably have produced similar results as the op-amps. The advantage of the op-amp is that a future version could easily increase the voltage levels so that a $150 \mathrm{Ohm}$ headset was just as loud or louder as a $300 \mathrm{Ohm}$ headset. 


\subsection{Conclusion}

Civilian and military aircraft headsets have evolved into two different connection and impedance standards that are incompatible. Needed, but not available, was a device that would allow for operators to use their favorite type of headset on any intercom.

This thesis reports on an intercom system which when tested, demonstrated full functionality while allowing for expandability of the intercom system. This design allows an operator to use whichever type of headset they choose, based on comfort or feature levels. This should help ensure satisfaction with the complete Project Oculus system.

In conclusion, an intercom system was designed, constructed, and tested that uses military and civilian headsets to communicate both internally for Project Oculus and externally to the C-130 crew and provides support for a headset to be connected externally of the operator station

\subsection{Future Work}

Time and budget constraints did not allow for certain items to be considered in this thesis. Testing of more headsets from different manufacturers with the impedance matching circuit would allow for more compatibility. The layout of the PCB (shown in Appendix A) could be much smaller and more efficiently laid out. The use of surface mount technology and smaller components would reduce board size. A loud speaker with proper electrical connections needed implemented and installed. Finally, radio switches need designed and installed to accommodate multiple radios for each person in the operator station. 


\section{Works Cited}

[1]. The 20th Century's Greatest Airlifter Will Also Be the 21st Century's Greatest. http://www.lockheedmartin.com. 2004.

[2]. Wowczuk, Zenovy, "Design of a Standardized Sensor Platform for a C-130 Aircraft", Thesis, August 2004. West Virginia University, C-130 Project Oculus.

[3]. Feragotti, Lawrence A., "Sensitiviy Analysis of the C-130 Sensor Deployment System Arm using the Finite Element Method", Thesis, 2004, West Virginia University, C-130 Project Oculus.

[4]. Naternicola, Adam. "Dynamic Modal Analysis and Optimization of C-130 Project Oculus' Mechanical/Pod Sensor Deployment System using Finite Element Method", Thesis, May 2004, West Virginia University, C-130 Project Oculus.

[5]. Shawlee, Walter. "Avionics System Design: Voodoo Audio". http://www.aviationtoday.com.

[6]. Hayes, Robert. "Control System design for a C-130 Ro-Ro Sensor Deployment Platform”, Thesis, May 2004, West Virginia University, C-130 Project Oculus.

[7]. Communication/Navigation Systems \& Specifications. http://www.aviationnow.com.

[8]. Northern Airborne Technology AA83-001 Inter Music Stereo Intercom Data Manual. April 2004, http://www.northernairborne.com.

[9]. Northern Airborne Technology AA36-100 Digital Tie Line Adapter Data Manual. November 2003, http://www.northernairborne.com.

[10]. National Semiconductor LM358. http://www.national.com. March 2004.

[11]. National Semiconductor LM386 Information page. http://www.national.com. August 2000.

[12]. National Semiconductor LM340 Information page. http://www.national.com. August 2004.

[13]. "Impedance Matching: A Primer. Electus Distributin”, 2001. http://www.electusdistribution.com.au. 
[14]. Phoenix Contact terminals specifications. http://eshop.phoenixcontact.com. June 2003

[15]. E-Switch specifications. http://www.e-switch.com.

[16]. Dialight LED datasheet. http://www.dialight.com.

[17]. Hirose HR10A-10R-10S Data Sheet. http://www.hirose.co.jp.

[18]. Flightcom Denali ANR Headset Specifications. http://flightcom.net. 


\section{Appendix A Custom Impedance Matching Circuit}

\section{A.1 Headset Impedance Matching Circuit Design}

Impedance matching of speakers can be achieved by two different methods. An impedance matching transformer can be placed between the amp and the speaker or the signal may be buffered by an amplifier which changes the impedance. The disadvantage of using the transformer is that low frequencies can be attenuated, which degrades audio signals. Amplifiers require power, and a bit of external circuitry, but don’t necessarily filter frequencies. Each of the methods has advantages and disadvantages, but for this design the amplifier method was chosen. The goal was to have a somewhat flat frequency response from $20 \mathrm{~Hz}$ to $20 \mathrm{kHz}$ and to match impedances with an op-amp (Operational Amplifier).

Transformers provide a passive component that does not require power or control. They also can be physically large and heavy mainly because they require many windings around an iron core. Transformers are rated by the number of turns, what type and size of wire, and the size of the iron core. Many different transformers were found that would possibly allow for passive impedance matching, but they were too large, and heavy. Transformers also have a specific frequency response, which if not matched to the application could be a hindering factor. 


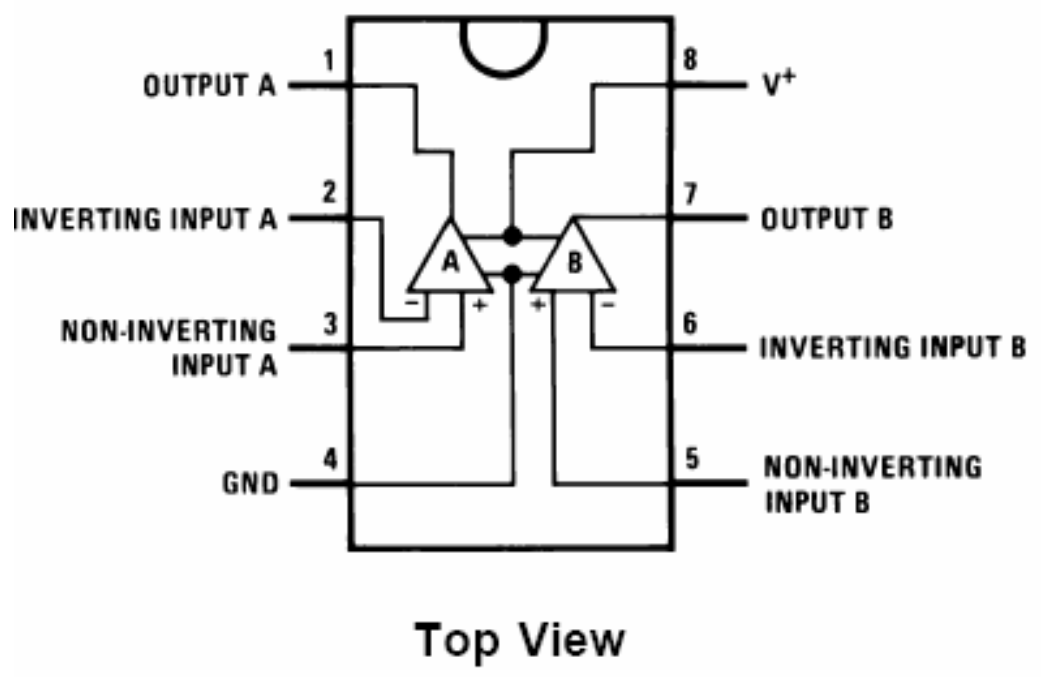

Figure 61 - LM358 Dual Op-Amp

For a dual op-amp situation such as the LM358 [10], the output impedance is very low, which eliminates the need for impedance matching. Therefore buffering an audio signal is a much better method of matching impedances because the components are not expensive and very small. Although this method is active and requires a power supply, it is much simpler to customize for a specific application than to create a transformer. In this case a very popular amplifier produced by National Semiconductor, the LM358 (Figure 61) was chosen. This small 8-pin DIP package allows for the use of a single rail power supply, meaning that only a ground and positive voltage were required instead of a ground, positive and negative power supply.

The LM358 contains two amplifiers in one package. Both of the amplifiers were used to buffer the right channel (Figure 62) coming from the NAT AA83 speaker output which has a source impedance of $300 \mathrm{Ohms}$. Because two amps are contained on a single chip, both were used for two reasons. First it was preferable to avoid the possibility of driving a commercial headset and a military headset at the same time from a single amplifier. This would only occur if the user was to plug both a civilian and military 
headset at the same time in a single amplifier situation. If this was to happen then the impedance would not be match, most likely drop significantly, and the amount of current required to drive more speakers could damage the amplifier. Second the addition of parts was minimal.

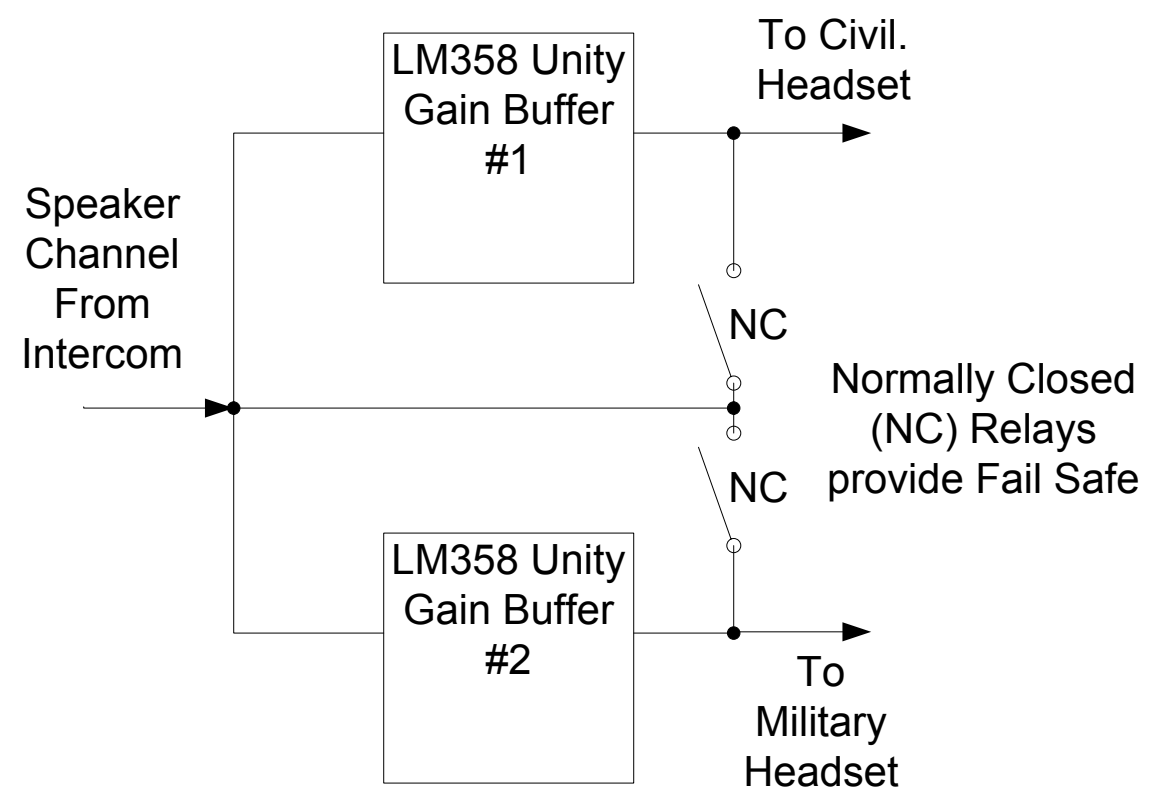

Figure 62 - Headphone Buffer Block Diagram

Single power supply op-amps that amplify audio signals, which are AC, require that the input be biased by a DC source. This is necessary because the single supply opamp cannot amplify the negative part of the AC signal, only the positive. This was achieved by biasing the input, after blocking the DC components of the signal, with a large capacitor to half of the supply voltage. Also the single supply op-amps have an output that is biased. To combat this to be able to drive a speaker, which is AC, a large capacitor was placed on the output.

The gain or input voltage divided by the output voltage of the LM358 was to be of value of one, which makes the op-amp a unity gain buffer. The biased input connects to the positive input of the op-amp and the negative input receives the un-altered feedback from the output. 


\section{A.1.1 Schematic of Headphone Buffer}

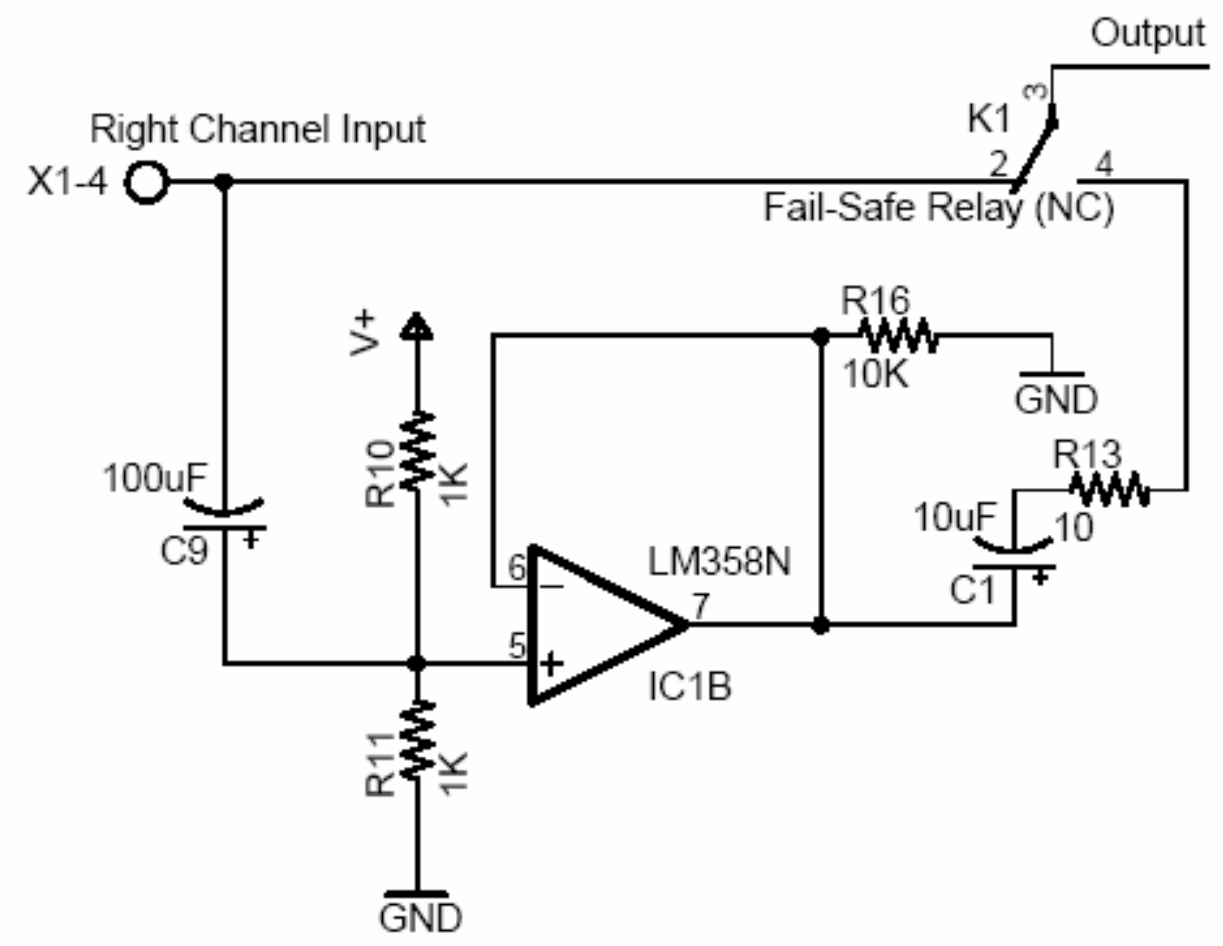

Figure 63 - Headphone Buffer Schematic

\section{A.2 Microphone Amplifier}

\section{A.2.1 Microphone Amplifier Description}

Dynamic microphones output a very small voltage, about $2.5 \mathrm{mVrms}$. The NAT

AA83 requires that the input voltage be around 500mVrms. The dynamic microphone then requires an amplifier to have a voltage gain of 200, which is very large. National Semiconductor produces a general purpose amplifier that contains a built in gain that can vary from 20 to 200 and requires only a single rail supply. This small 8-pin DIP package chip is the LM386 (Figure 64) [11]. Because the gain is internal, the noise is minimized and the numbers of components are decreased. 


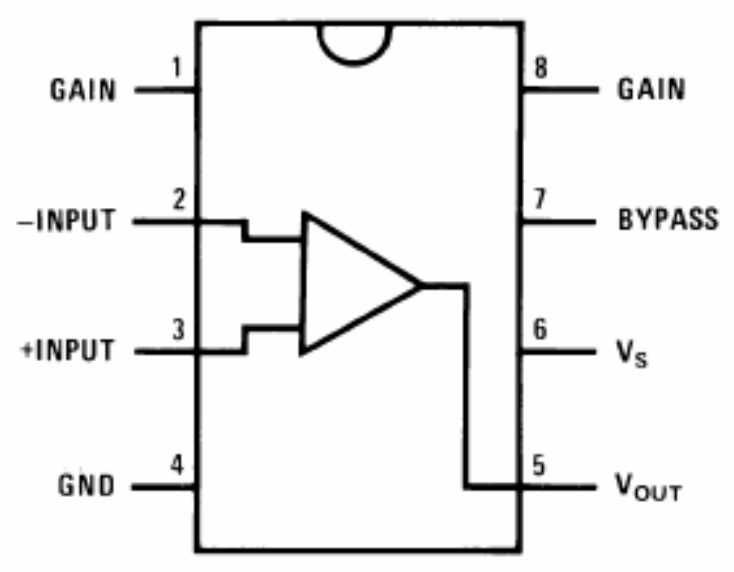

Figure 64 - LM386 Low Voltage Power Amplifier

In addition the NAT AA83 expects the microphone impedance to be, about 150 ohms, which is much larger than the $5 \mathrm{ohm}$ impedance of the dynamic microphone. The most important part is this circuit is to have a correct signal level for the input on the NAT AA83. Impedance is almost not an issue because nothing is being driven from the signal and it is at a significant voltage level.

The microphone circuit (Figure 65) must support three separate microphones and a switching circuit must be used to control which signal is selected as an output. Even though it would be possible to connect the three signals together to form an output it is not advisable. If more than one signal is connected, impedances will not match and energy would flow between the sources creating problems. Also when the dynamic microphone is not in use its output from the amplifier should be switch off for noise reasons.

The switching circuit was constructed using three relays and a three position switch. A single relay is selected from one of the three by the switch and it is activated to select its source as the output. A fail safe situation needed to exist where if power failed the microphone signals would pass through the device. This was accomplished by connecting the relays with their normally closed position. Once powered up the circuit 
opens the relays that are not to be used, and shuts off power to the relay that is selected as the source which causes the relay to default to the closed state. Figure 66 shows the connection paths of the relays and Figure 67 displays the control circuit for the LED's and relays based on the switch.

\section{A.2.2 Schematic of the Microphone Circuit}

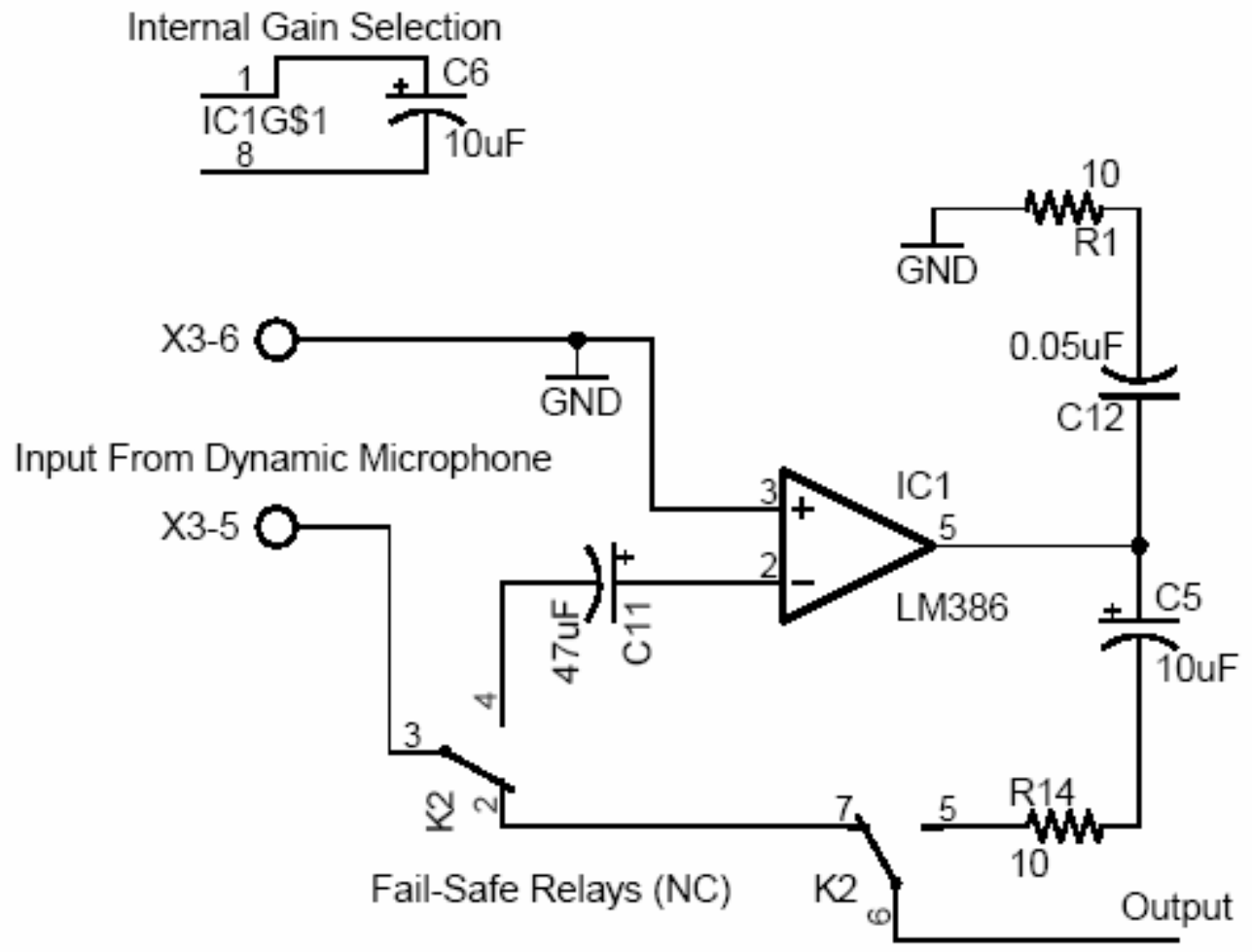

Figure 65 - Dynamic microphone amplifier Circuit 


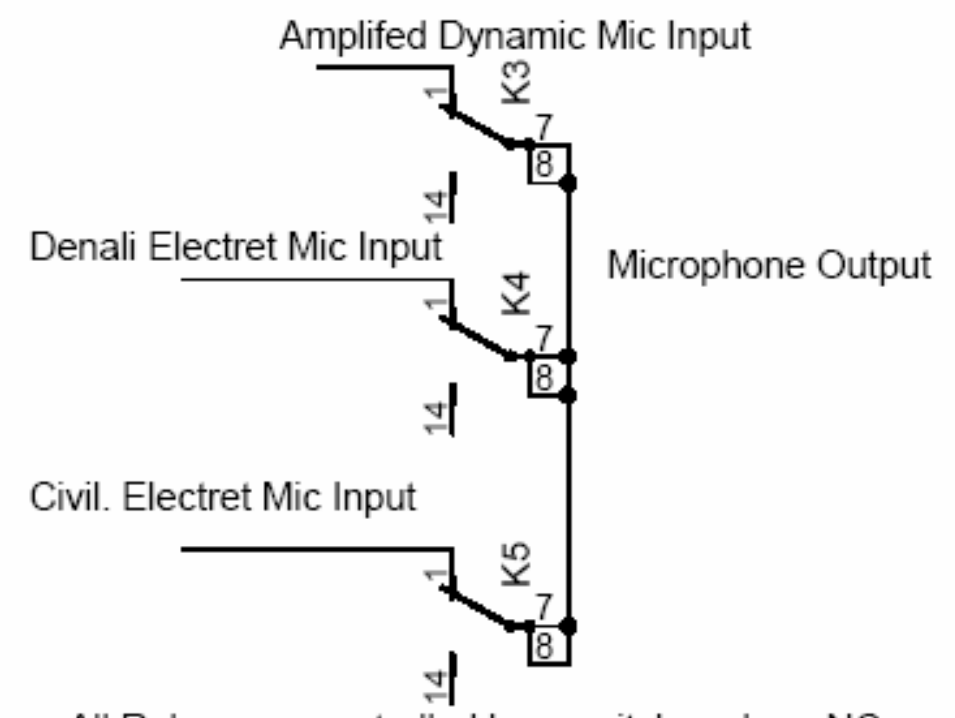

All Relays are controlled by a switch and are NC

Figure 66 - Microphone Relay Selection Circuit

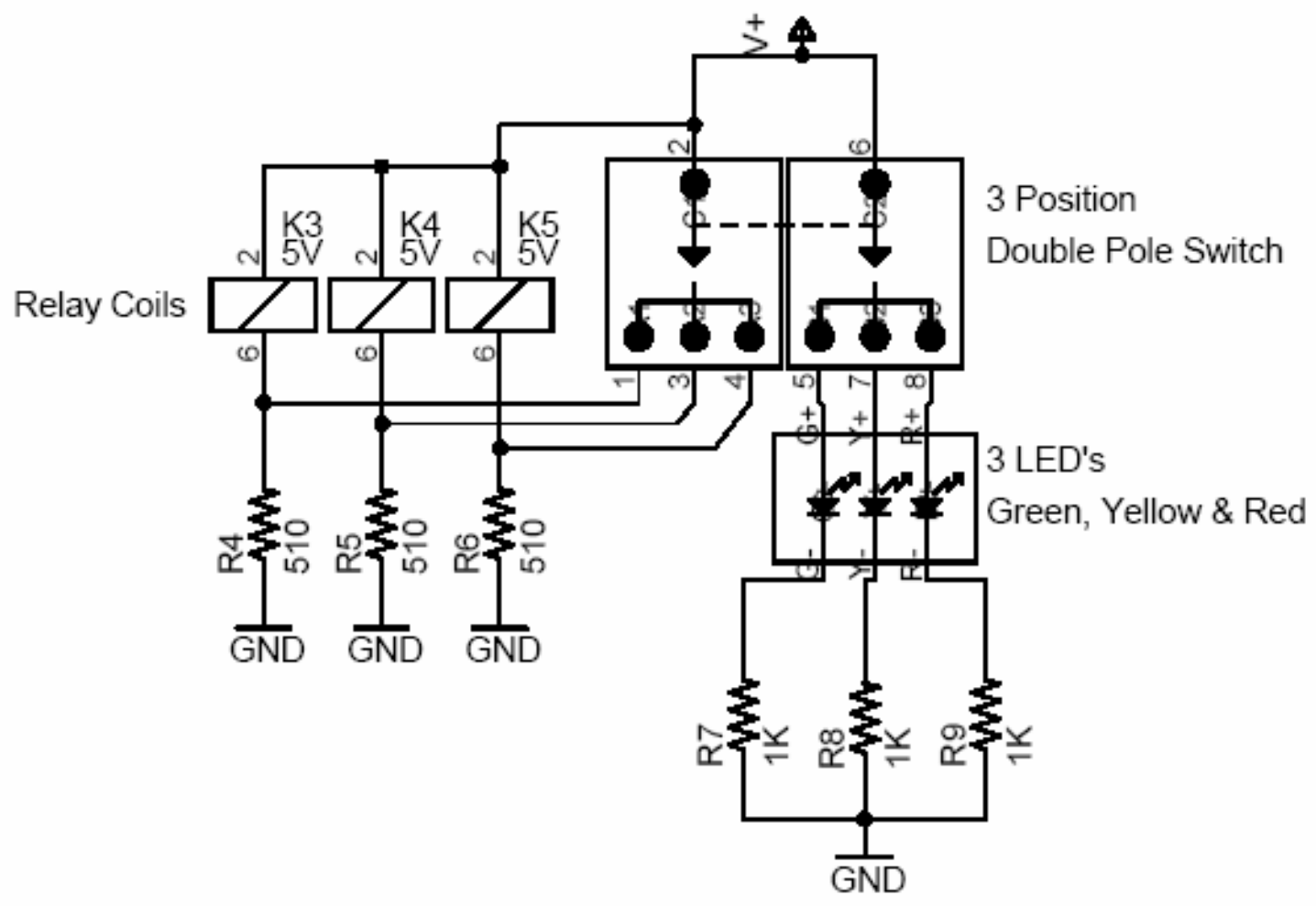

Figure 67 - Microphone Relay Control Selection Circuit 


\section{A.3 Complete Circuit Description}

Combining the headset speaker buffer and the dynamic microphone into a single circuit provides the NAT AA83 a way to communicate with an expanded range of headsets. In addition to the two small circuits the entire circuit needed a power supply, a fail-safe relay system, LED indicators, selection switch and connection terminals.

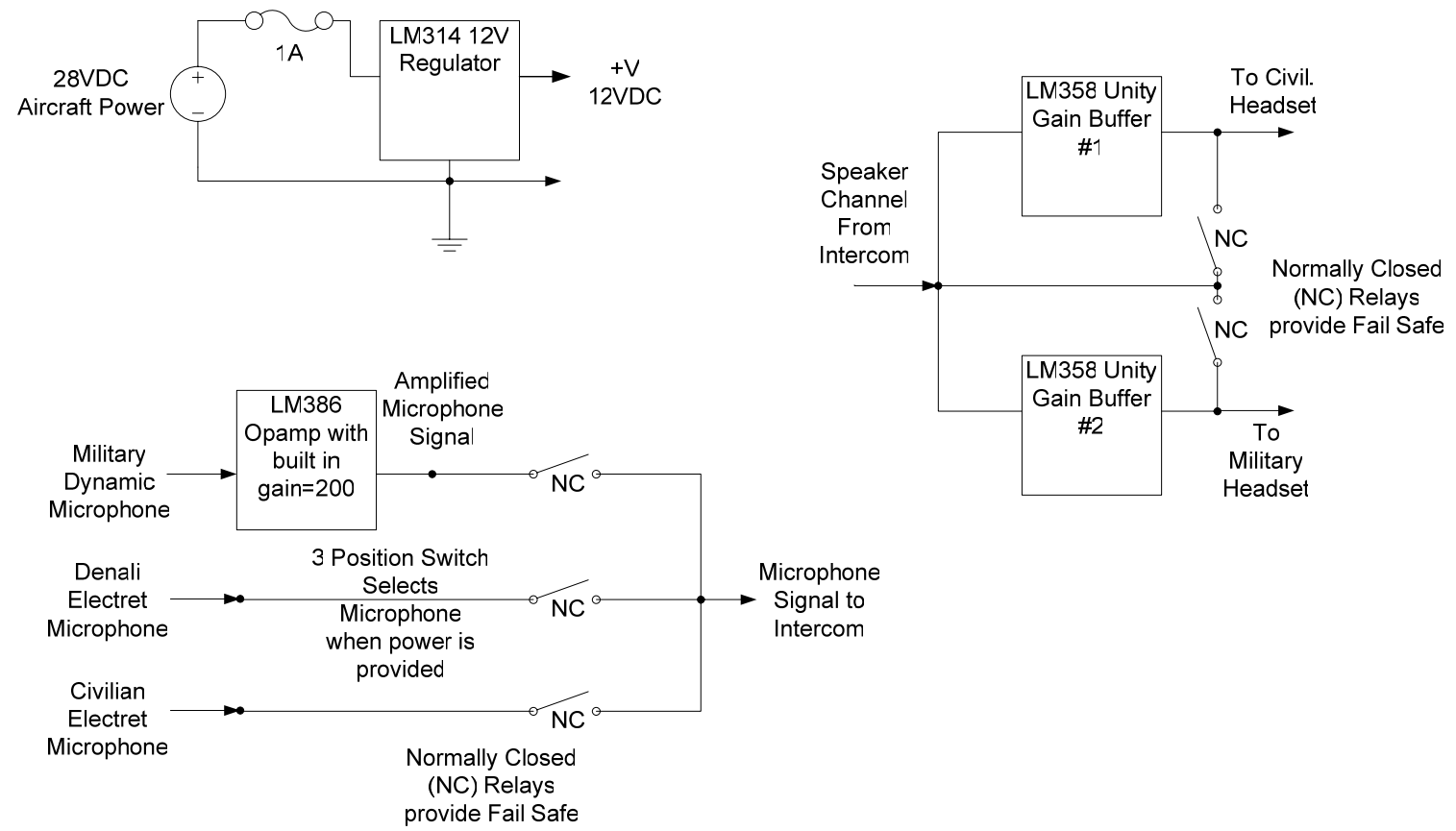

Figure 68 - Active Impedance Matcher Block Diagram 


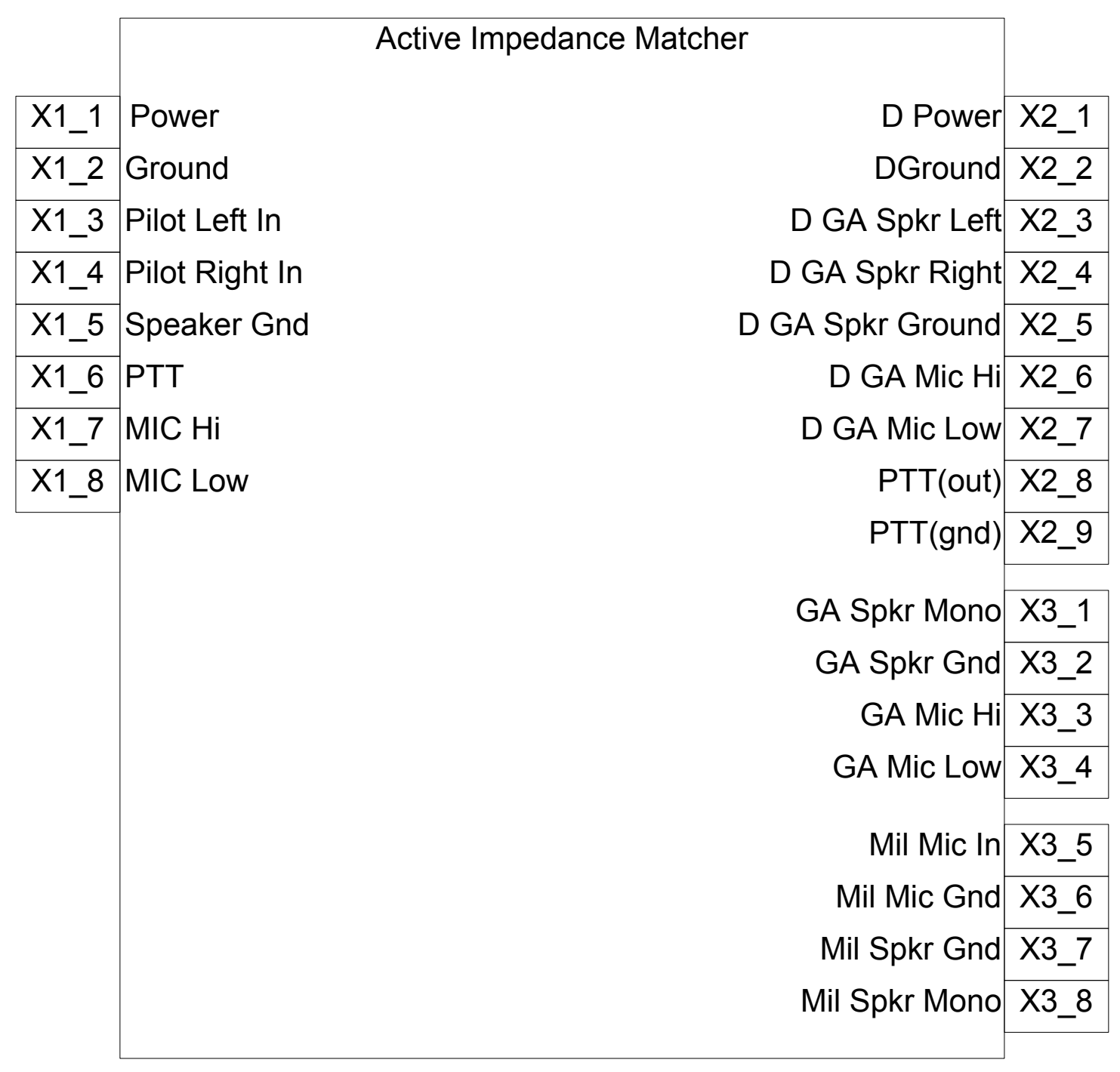

Figure 69 - Block diagram of inputs and outputs

The power supply available to Project Oculus in the C-130 aircraft is either $28 \mathrm{VDC}$ or three phase $208 / 110 \mathrm{VAC} 400 \mathrm{~Hz}$. The AC is eliminated as a power supply because the relays, op-amps and LED's operate on DC and it would be costly and wasteful to include another power converter. $28 \mathrm{VDC}$ is too high for the majority of the components chosen for the board, so either a voltage regulator or DC to DC converter was necessary. DC to DC converters are large compared to voltage regulators, usually require inductors, and have switching noise associated with the conversion but are 
extremely efficient. Most of these traits are unwanted on a small PCB that is meant for noise free audio. A brute force, linear voltage regulator was chosen, even though they have heat dissipation issues while conducting large amounts of current. The single chip three pin LM340-12 [12] provides sufficient voltage regulation of 12VDC at a maximum input of 35VDC and maximum output current of about $2 \mathrm{~A}$. The entire circuit of the impedance matching uses approximately $200 \mathrm{~mA}$ at the worst case. The LM340-12 at the highest PCB load will burn off approximately $3.2 \mathrm{~W}$ of heat in the worst case, which is easily taken care of by the heat sink on the PCB and the extra ground trace on the PCB meant just for heat dissipation.

Power, in the form of 9-32VDC, must be provided to the Flightcom Denali ANR (Active Noise Reduction) headset in order for the ANR to work. This is accomplished in either one of two methods, both of which connect to the Impedance Matching PCB. The selection is achieved by a jumper on the board which will select one or the other methods. First power can be drawn as 28VDC from a shared 1A fuse with the impedance matching board. This allows the LM340-12 to save on heat dissipation. Second the power can come from the $12 \mathrm{VDC}$ which is regulated by the LM340-12. Either way is acceptable and can be configured by the user. 


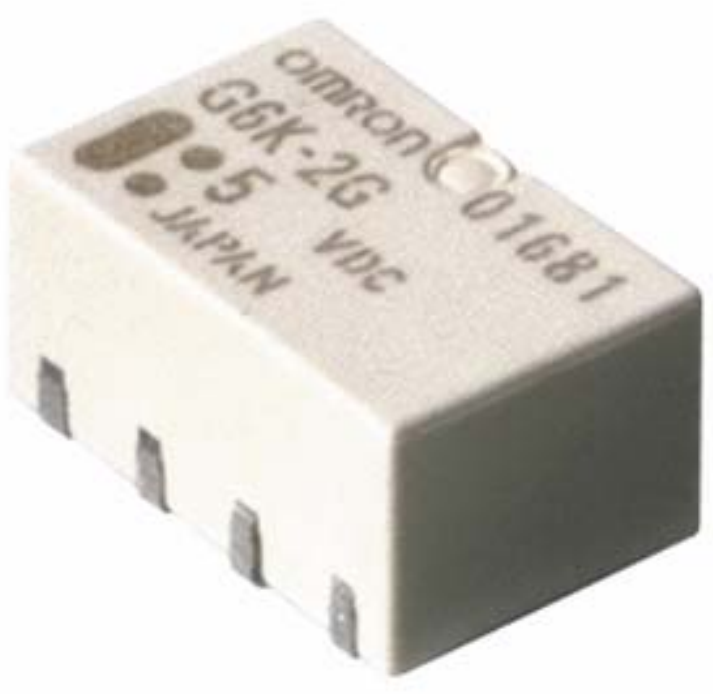

Figure 70 - PCB Relay

Circuitry in aircraft always needs a fail-safe backup system. If a system in aircraft fails it could easily be life threatening. The fail-safe system on the circuit utilizes relays (Figure 70) to bypass the op-amps and directly connect the inputs with the outputs. This is accomplished by using the property of a relay called normally closed. A relay consists of two parts, a coil and contacts. The contacts are nothing more than a switch with typically two poles, one called normally open, the other normally closed. When the energy is lost or disconnected the contact returns back to its normally closed position. When the coil is energized the contacts close to the normally open state. This system provides a fail-safe that if power is lost to the impedance matching circuit the microphone and speaker signals will pass through the device, although they will be at low levels.

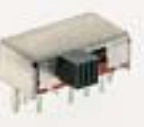

Figure 71 - E-Switch 3 Position 2315 [15] 


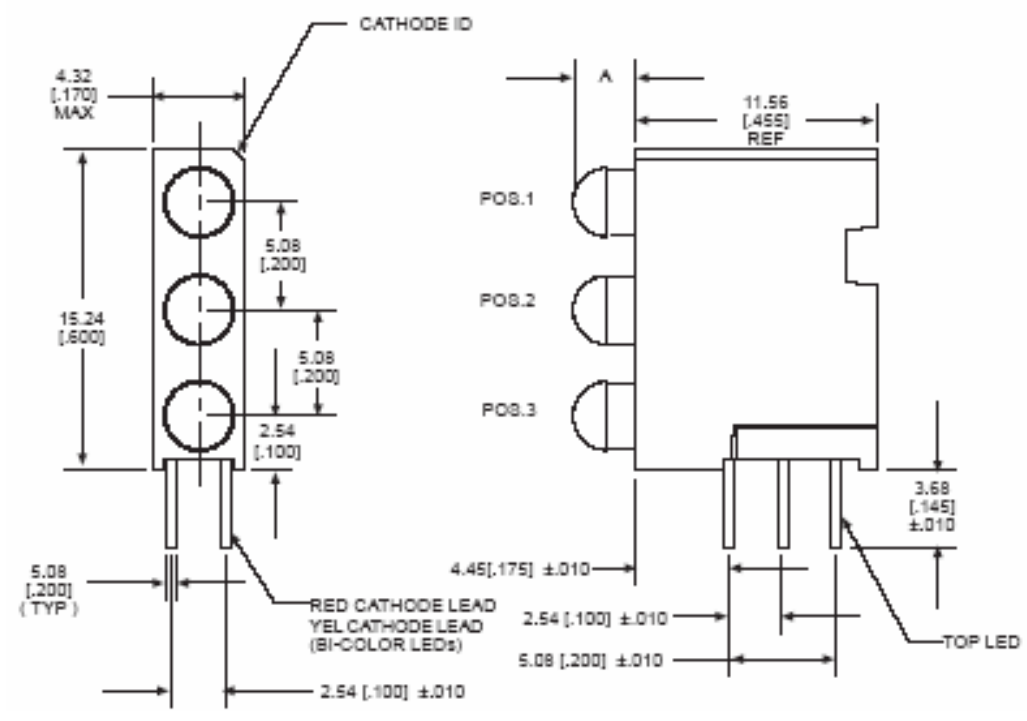

Figure 72 - Dialight 3mm Tri-Color LED [16]

LED indicators and a switch were necessitated to select which microphone input source was in use for several reasons. First the necessary elimination of a noise source through the system by turning off the output of the LM386 op-amp when not used. Second, if for some reason the operator has more than one type of headset connected simultaneously to the circuit then a choice needs to be made as to which microphone is used or else the system may become unusable. Utilizing the fail-safe relays, the inputs from the microphones were connected in a special fashion to allow selection of one of the three from a switch. The switch can be seen as Figure 71, which is a low profile 3 position double pole switch. The LED used to show status can be seen in Figure 72, which is three LED's stacked on top of each other to save PCB space. According to the Dialight LED's datasheet, the voltage drop across the diode is $2.1 \mathrm{~V}$ for green and yellow and $2 \mathrm{~V}$ for red and $10 \mathrm{~mA}$ for each LED. Calculating the resistor value of $1 \mathrm{kOhm}$ was determined by using a source voltage of $12 \mathrm{~V}$, drop across the LED of $2 \mathrm{~V}$, and current as $10 \mathrm{~mA}$, then rounding up the result. The larger resistor value only makes the LED a little 
dimmer, but is safer because it restricts more current and allows for an error in values of the LED and resistors.

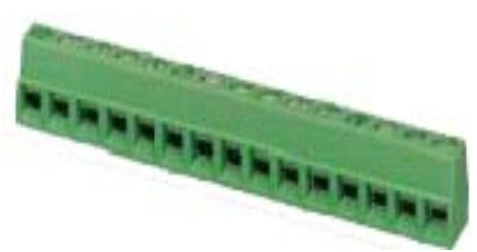

Figure 73 - Multi Connector Phoenix Contact Block [14]

Connection terminals are a very important part of any circuit. Normally, some type of terminal would be used that were quick and easy to connect wires and simple to replace the entire board without disturbing many connections. In this case, three screw connection terminals with ten connections each were chosen to support all of the inputs, outputs and power supply because of the need for reliability.

PTT (Push to Talk) is a method of pressing a button when the user wants to talk into the system. Pressing a button ensures the users that useless background noise will not be transmitted on the intercom at all times. Included on the impedance matching board is a pass-through of the PTT signals from the NAT AA83. This was done to make wiring to the NAT AA83 simple because all the wires from it go into the impedance matching board and no wire can bypasses the impedance matcher. 


\section{A.4 Schematic and Layout of Impedance Matching Circuit}

\section{A.4.1 Schematic}

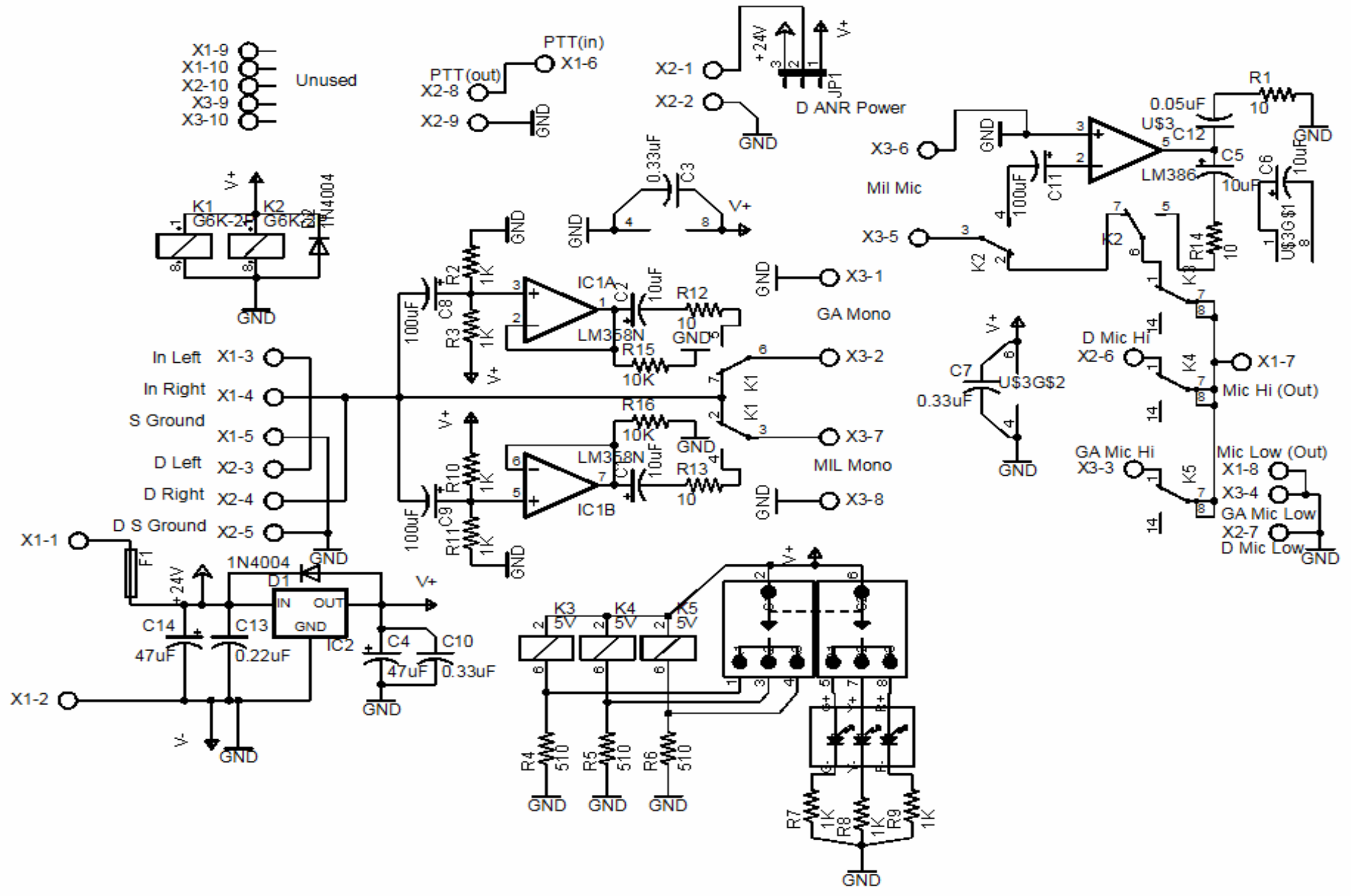

Figure 74 - Schematic as Drawn in Eagle PCB 


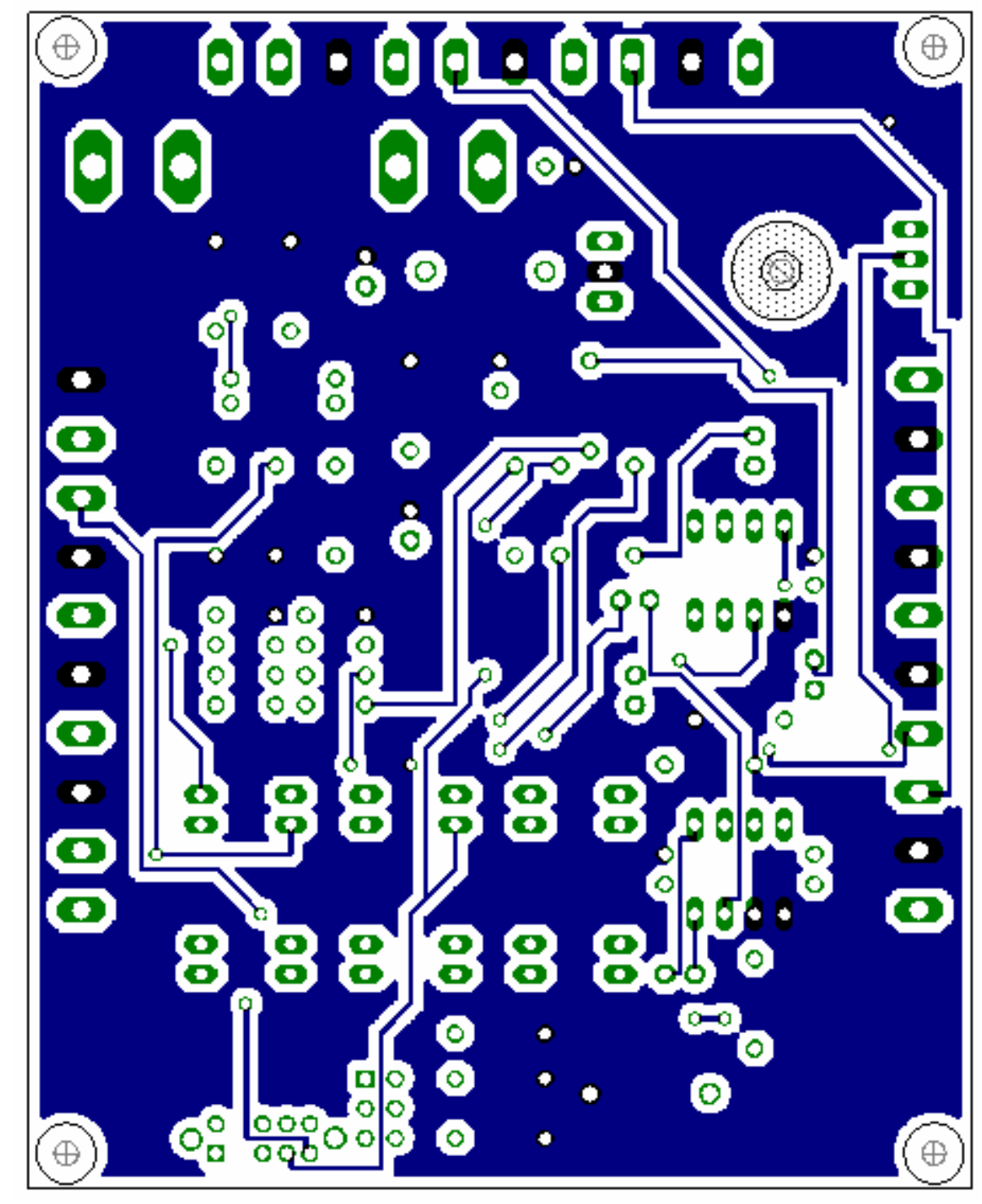

Figure 75 - Bottom Layer of PCB

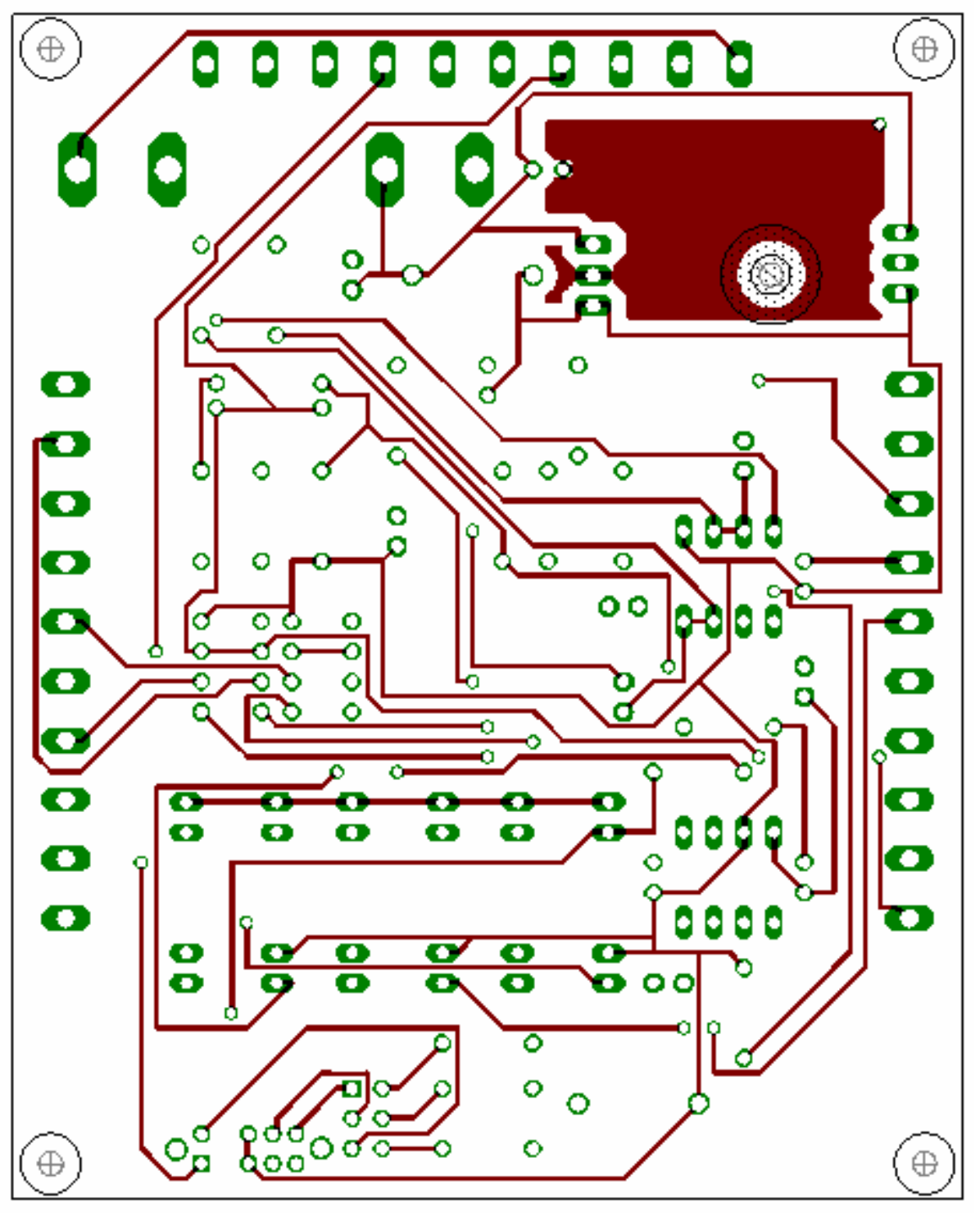

Figure 76 - Top Layer of PCB 


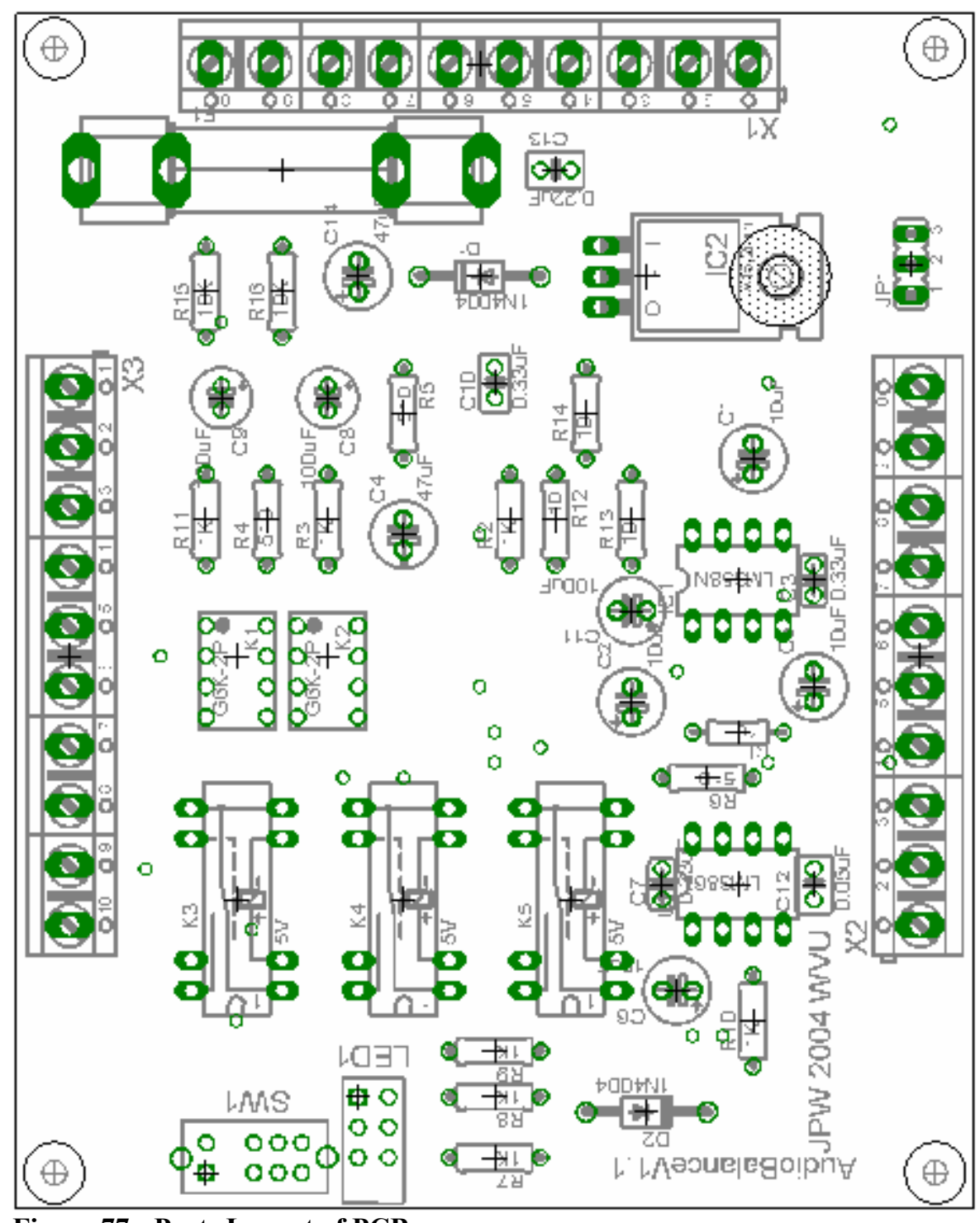

Figure 77 - Parts Layout of PCB 


\section{A.5 List of Inputs and Outputs}

\begin{tabular}{|l|l|}
\hline $\mathrm{X} 1 \_1$ & Power In (28VDC) \\
\hline $\mathrm{X} 1 \_2$ & Ground \\
\hline $\mathrm{X} 1 \_3$ & Left Headphone In \\
\hline $\mathrm{X} 1 \_4$ & Right Headphone In \\
\hline $\mathrm{X} 1 \_5$ & Headphone Ground \\
\hline $\mathrm{X} 1 \_6$ & PTT in \\
\hline $\mathrm{X} 1 \_7$ & Microphone Hi Out \\
\hline $\mathrm{X} 1 \_8$ & Microphone Low Out \\
\hline $\mathrm{X} 1 \_9$ & N/C \\
\hline $\mathrm{X} 1 \_10$ & N/C \\
\hline
\end{tabular}

Table 1 - X1 Connections

\begin{tabular}{|l|l|}
\hline $\mathrm{X} 2 \_1$ & Denali Power Positive (Fused) \\
\hline $\mathrm{X} 2 \_2$ & Denali Power Ground \\
\hline $\mathrm{X} 2 \_3$ & Denali Headphone Right \\
\hline $\mathrm{X} 2 \_4$ & Denali Headphone Left \\
\hline $\mathrm{X} 2 \_5$ & Denali Headphone Ground \\
\hline $\mathrm{X} 2 \_6$ & Denali Microphone Hi \\
\hline $\mathrm{X} 2 \_7$ & Denali Microphone Low \\
\hline $\mathrm{X} 2 \_8$ & PTT (out) \\
\hline $\mathrm{X} 2 \_9$ & PTT Ground \\
\hline $\mathrm{X} 2 \_10$ & N/C \\
\hline
\end{tabular}

Table 2 - X2 Connections

\begin{tabular}{|l|l|}
\hline X3 31 & Civil Headphone \\
\hline X3_2 & Civil Headphone Ground \\
\hline X3_3 & Civil Microphone Hi \\
\hline X3_4 & Civil Microphone Low \\
\hline X3_5 & Military Microphone \\
\hline X3_6 & Military Microphone \\
\hline X3_7 & Military Headphone \\
\hline X3_8 & Military Headphone Ground \\
\hline X3_9 & N/C \\
\hline X3 310 & N/C \\
\hline
\end{tabular}

Table 3 - X3 Connections 


\section{A.6 Parts list for PCB}

\begin{tabular}{llll}
\hline Part & Description & Manufacturer & Manufacturer Part \# \\
\hline IC2 & 12 V Regulator & National Semiconductor & LM340T-12 \\
U\$3 & LM386-4 Amp & National Semiconductor & LM386N-4 \\
IC1 & LM358P Amp & Texas Instruments & LM358P \\
K3,K4,K5 & 5V Reed SPDT & Coto Technology & 8L41-05-011 \\
K1,K2 & 12V Relay DPDT & Omron & G6K-2P DC12 \\
R15,R16 & 10K Resistor & Yageo & CFR-25JB-10K \\
R4,R5,R6 & 510 Resistor & Yageo & CFR-25JB-510 \\
R1,R12,R13,R14,R15 & 10 Resistor & Yageo & CFR-25JB-10 \\
R2,R3,R7,R8,R9,R10,R11 & 1K Resistor & Yageo & CFR-25JB-1K \\
SW1 & 3 Position Switch & E-Switch & EG2315 \\
C1,C2,C5,C6 & 10 uF Electrolytic Cap & Nichicon & UVZ1V100MDD \\
C4,C8,C9,C11,C14 & 47uF Electrolytic Cap & Nichicon & UVZ1V470MDD \\
C3,C7,C10,C12,C13 & 0.33 uF & Panasonic & ECS-F1VE335K \\
LED1 & 3 Color LED & Dialight & 564-0100-132 \\
X1,X2,X3 & 10pin Screw Terminal & Phoenix Contact & 1729092 \\
F1 & 1A Fuse & Littelfuse & 0312001.H \\
F1 & Fuse Holder for PCB & Littelfuse & 01020079H \\
Accessory & 8Pin DIP Socket & Mill-Max Manufacturing & 110-99-308-41-001000 \\
Accessory & Standoffs(0.75) 8-32 Screw & Keystone Electronics & 2218 \\
Accessory & \#8 Screw & Building Fasteners & PMS 832 0038 PH \\
Accessory & Heat Sink & WAKEFIELD ENGINEERING & 273-AB \\
Table 4 - List of parts on the PCB & &
\end{tabular}




\section{A.7 Assembled PCB}

The assembled PCB as shown in Figure 78 shows the assembled PCB. The

corners of the board have holes that are meant as mounting holes. The likely case for mounting would be to have a perpendicular board to the panel (Figure 83) that the boards can be mounted to so it does not come lose with vibration in the aircraft. For the schematic and top, bottom, and parts layout see Appendix A, Table 4.

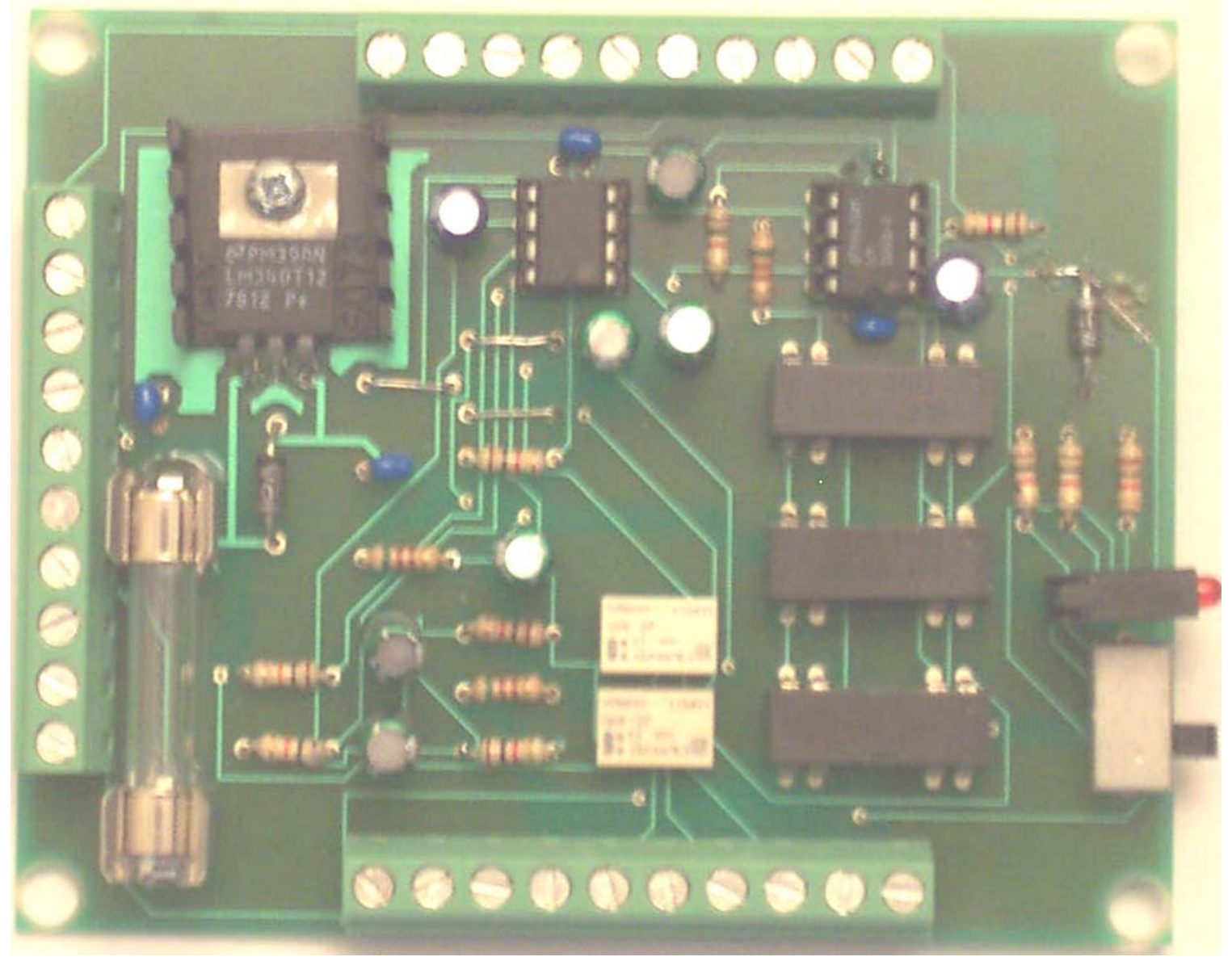

Figure 78 - Picture of Circuit Board

\section{A.8 Various Measured Frequency Responses and other Tests}




\section{Appendix B Project Oculus Intercom Schematics}

\section{B.1 Pilot and Co-Pilot Positions Schematic}

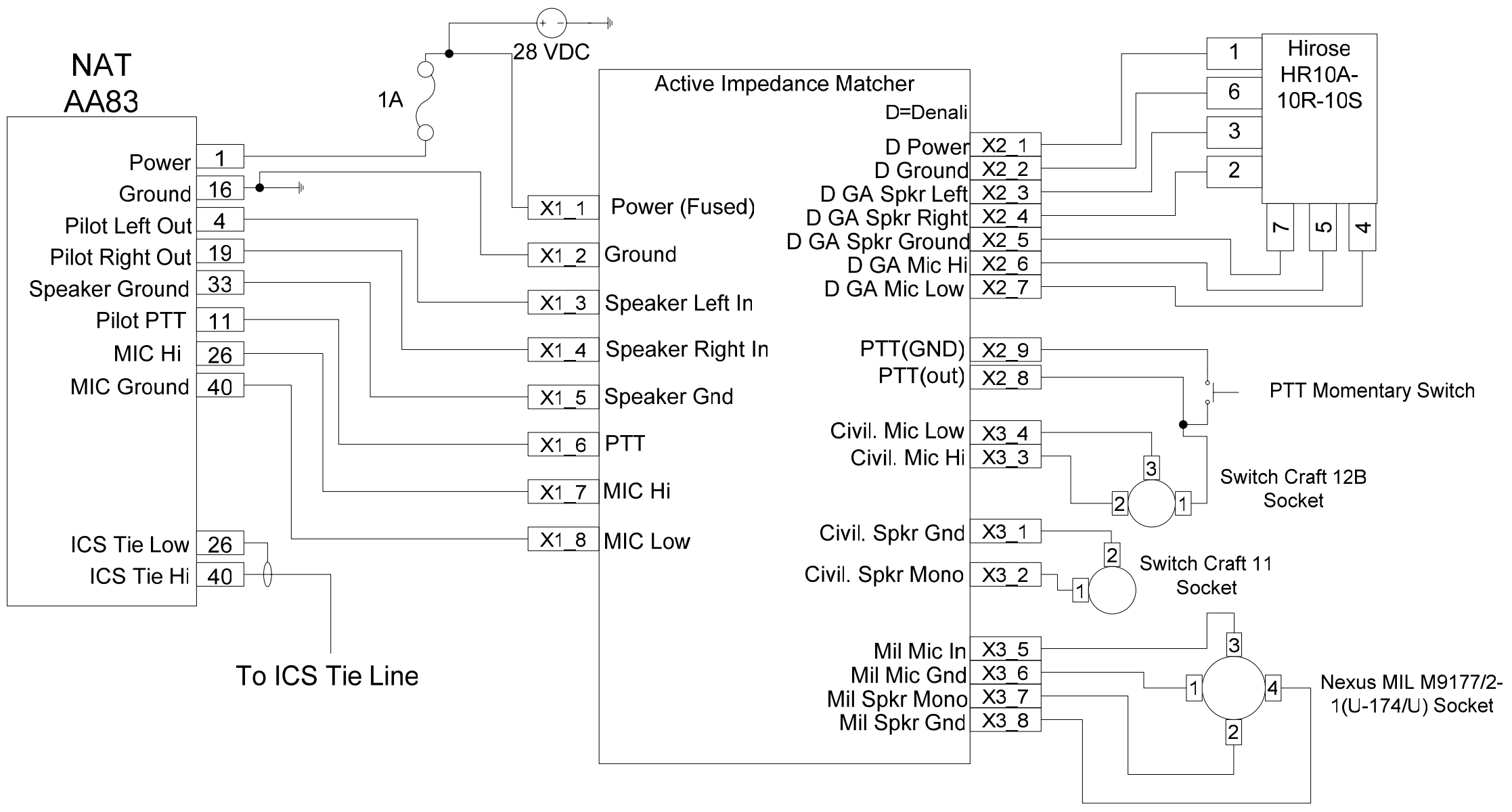

Figure 79 - Pilot/Co-Pilot Position Intercom Connection Diagram 


\section{B.2 Middle Position Schematic}

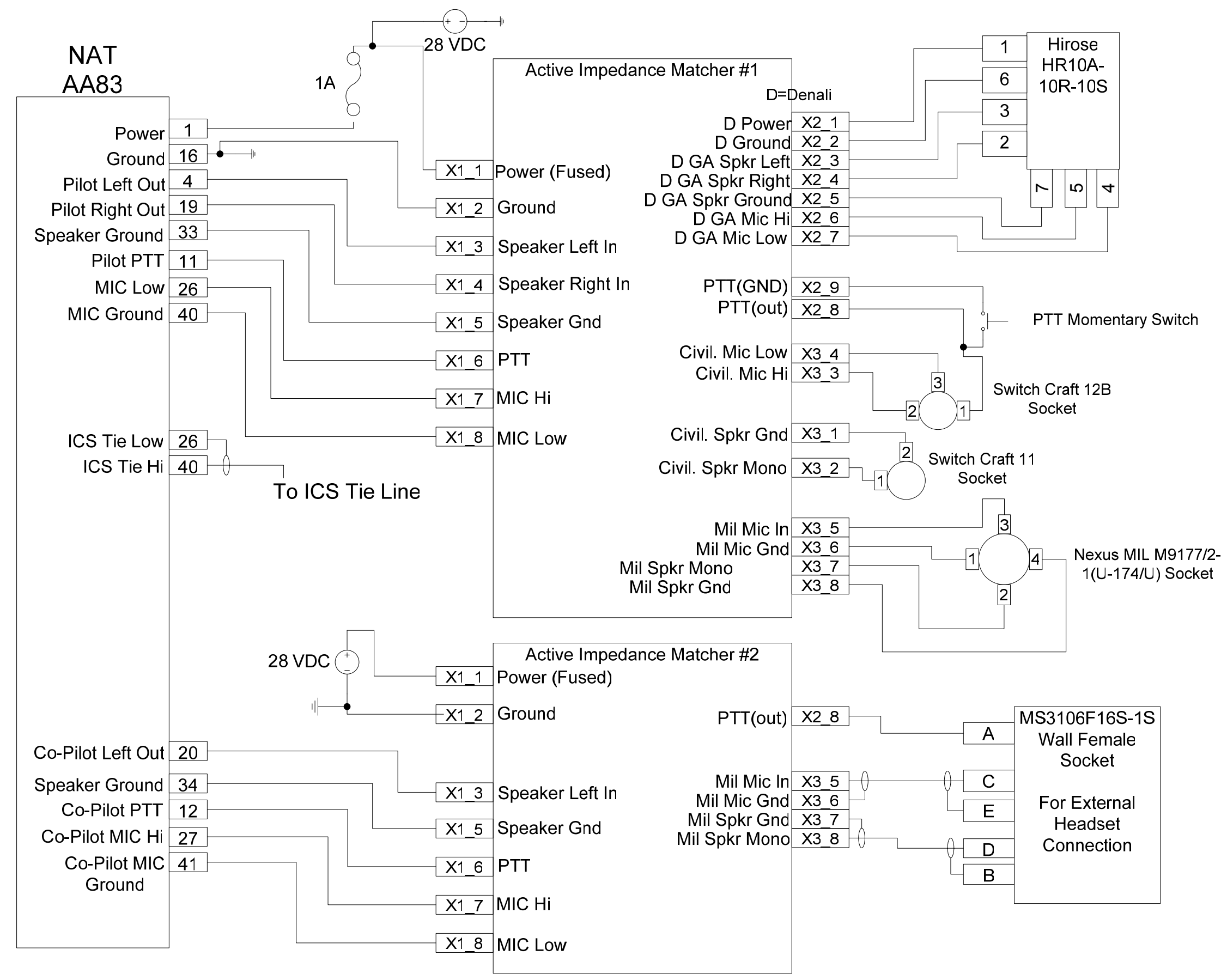




\section{B.3 Intercom Tie Line Connections}

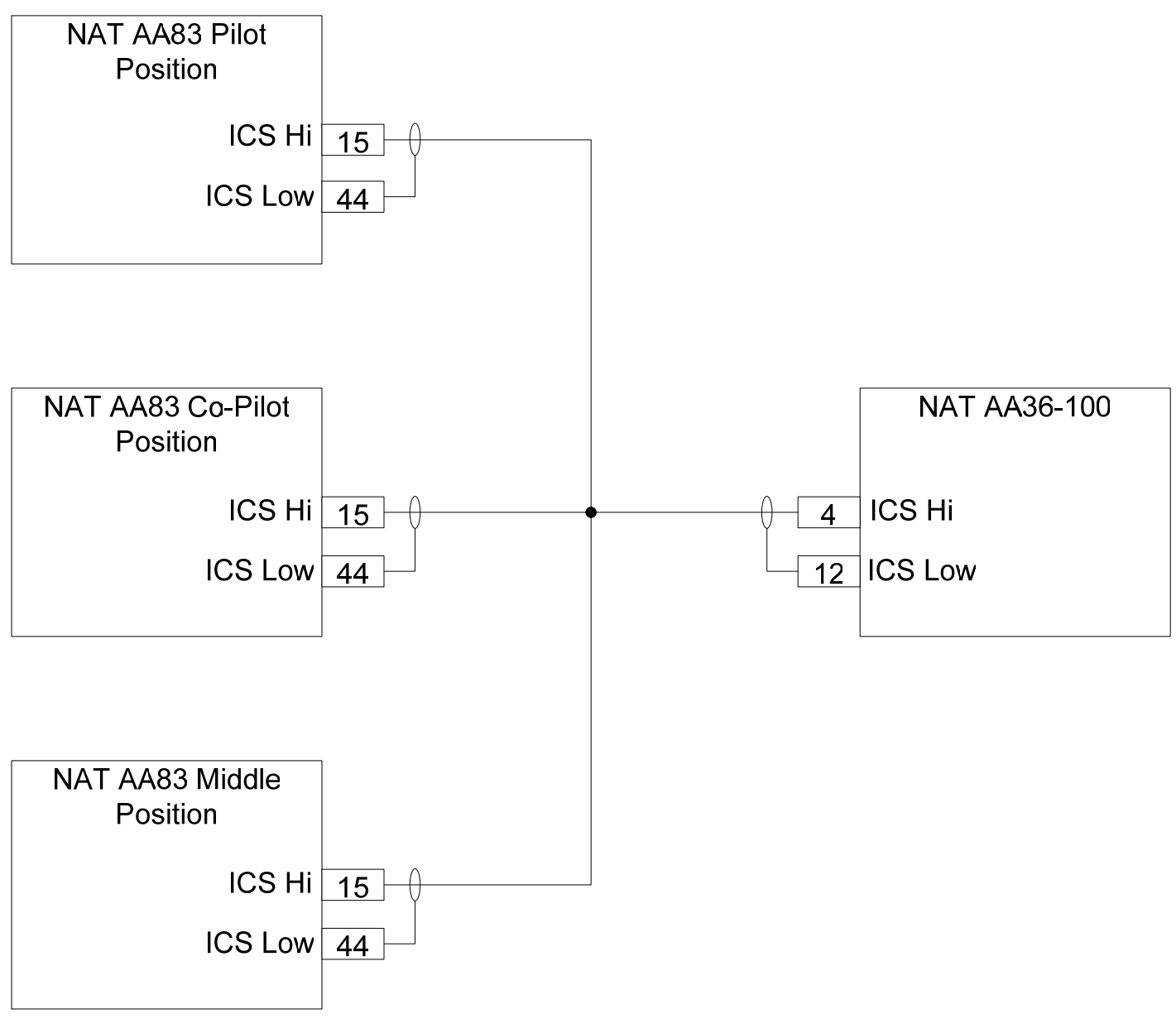

Figure 81 - Intercom Tie Line Connections 


\section{B.4 C-130 Intercom Connection}

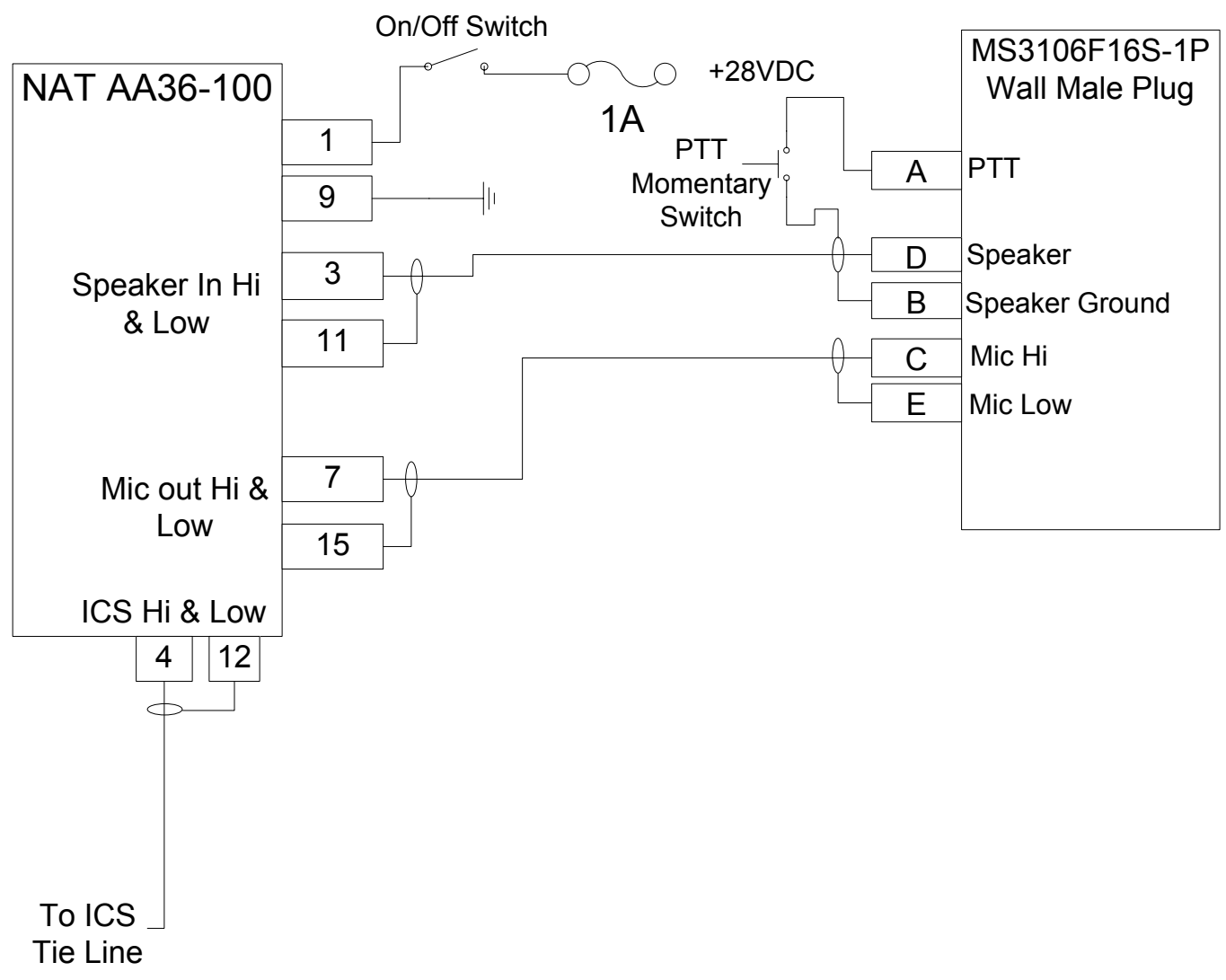

Figure 82 - Intercom Connection to the C-130 Intercom 


\section{B.5 Panel Layout of Project Oculus Intercom System}

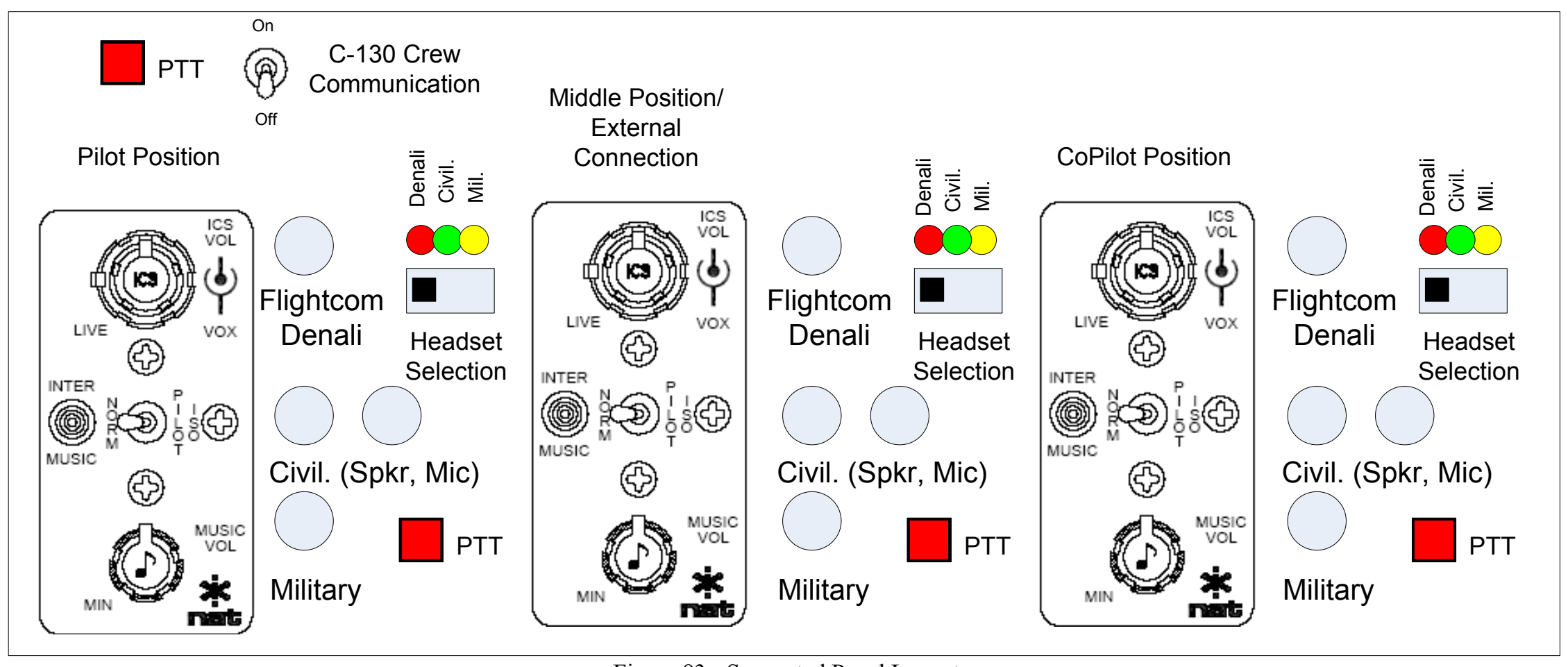

Figure 83 - Suggested Panel Layout 


\section{B.6 Parts List for Intercom System}

\begin{tabular}{|c|c|c|c|}
\hline Description & Manufacturer & Manufacturer Part \# & $\begin{array}{l}\text { Quantity } \\
\text { Needed }\end{array}$ \\
\hline $\begin{array}{l}\text { Denali Headset Plug } \\
\text { Cover }\end{array}$ & Hirose & HR10-10R-C & 3 \\
\hline GA Speaker Socket & SwitchCraft & & 3 \\
\hline GA Mic Socket & SwitchCraft & & 3 \\
\hline MIL Headset Socket & Nexus & TJT-102 & 3 \\
\hline DB44 Connector & Norcomp & 180-044-202-001 & 4 \\
\hline Case For DB44 & Norcomp & 972-025-01S-011 & 4 \\
\hline NAT AA83 & $\begin{array}{l}\text { Northern Airborne } \\
\text { Technology }\end{array}$ & AA83 & 3 \\
\hline DB15 Connector & Norcomp & 180-044-202-001 & 1 \\
\hline Case for DB15 & Norcomp & 972-025-01S-011 & 1 \\
\hline NAT AA36-100 & $\begin{array}{l}\text { Northern Airborne } \\
\text { Technology }\end{array}$ & AA36-001 & 1 \\
\hline PTT Switch & E-Switch & PS1023AT-RED & 3 \\
\hline Fuse Holder & LittelFuse & $01550100 Z$ & 5 \\
\hline 1A Fuse & LittelFuse & $0312001 . \mathrm{H}$ & 5 \\
\hline Single Pole Switch & Carling Tech. & 2FA53-73/TABS & 1 \\
\hline 7Pin Circular Wall Male & Amphenol & MS3102R16S-1P & 1 \\
\hline 7Pin Circular Wall Female & Amphenol & MS3102R16S-1S & 1 \\
\hline 7Pin Circular Plug Male & Amphenol & MS3456W16S-1P & 1 \\
\hline 7Pin Circular Plug Female & Amphenol & MS3456W16S-1S & 1 \\
\hline External Cable & Gavitt & WM-85/U M5898 & $60 \mathrm{Ft}$. \\
\hline $\begin{array}{l}\text { Active Impedance Box } \\
\text { (See Table } 4 \text { ) }\end{array}$ & Jay Wilhelm & N/A & 4 \\
\hline
\end{tabular}

Table 5 - Complete List of Intercom Parts

\section{B.7 Description of NAT AA83}

Northern Airborne Technologies produces an Intercom system entitled the

AA83 [8]. This device allows for multiple headset connections, a radio connection

and a Tie Line interface. The headset connections are of the Civilian type which

includes the $300 \mathrm{ohm}$ stereo speakers and an electret condenser microphone. Figure

84 displays the front panel where the controls of the AA83 reside.

Included on the AA83 is the feature called VOX (Voice Activation). This

feature allows the user to speak hands free. The user simply sets the threshold of activation. Instead of using a button to talk, this feature allows the user to use their 
hands. Although this feature is extremely useful, it sometimes cannot be used in exceptionally loud environments.

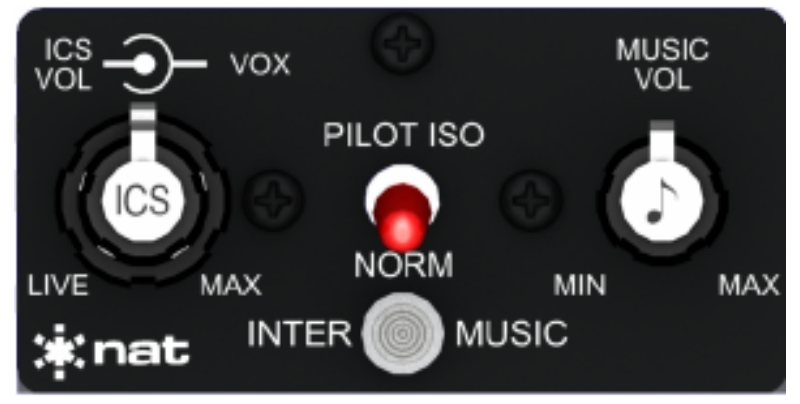

Figure 84 - NAT AA83 Front Panel

\section{B.8 NAT AA83 Specifications}

All of the specifications were taken from the NAT AA83 datasheet pg. 10-11

[8].

\begin{tabular}{|l|l|}
\hline Input Power (Voltage) & \\
\hline Nominal & 27.5 or 13.8 VDC \\
\hline Maximum & 30.3 VDC \\
\hline Minimum & 11.0 VDC \\
\hline
\end{tabular}

\begin{tabular}{|l|l|}
\hline $\begin{array}{l}\text { Microphone } \\
\text { Input }\end{array}$ & \\
\hline Level & $250 \mathrm{mVrms}(125$ to $500 \mathrm{Vrms})$ \\
\hline Impedance & $150 \mathrm{Ohm}+/-10 \%$ \\
\hline
\end{tabular}

\begin{tabular}{|l|l|}
\hline Speaker Output & \\
\hline Level & $5.5 \mathrm{Vrms}(100 \mathrm{~mW})$ \\
\hline Impedance & $300 \mathrm{Ohm}+/-10 \%$ \\
\hline Type & Transformer, unbalanced \\
\hline
\end{tabular}

Table 6 - AA83 Specifications 


\section{B.9 Description of NAT AA36-100}

The AA36-100 Digital Tie Line [9] adapter produced by Northern Airborne

Technologies allows for intercom integrations of different types. Many different impedance levels and voltage levels are supported by the device. It includes ten settings for different tie lines, headsets and microphones. The main purpose for use in Project Oculus is to broadcast the tie line, which the AA83 uses to communicate, to the $\mathrm{C}-130$ intercom system. The settings are determined by dialing in the correct settings via the datasheet. Headset signals are actually inputs to the AA36-100, which it broadcasts on the tie line. Microphone signals are sent to the $\mathrm{C}-130$ intercom via the tie line. This is a fully digital device, which means there is no analog circuitry connecting the tie line to the $\mathrm{C}-130$ intercom, just sampling and broadcasting. Figure 85 shows the enclosure and Figure 86 shows the locations of the settings of the AA36-100.

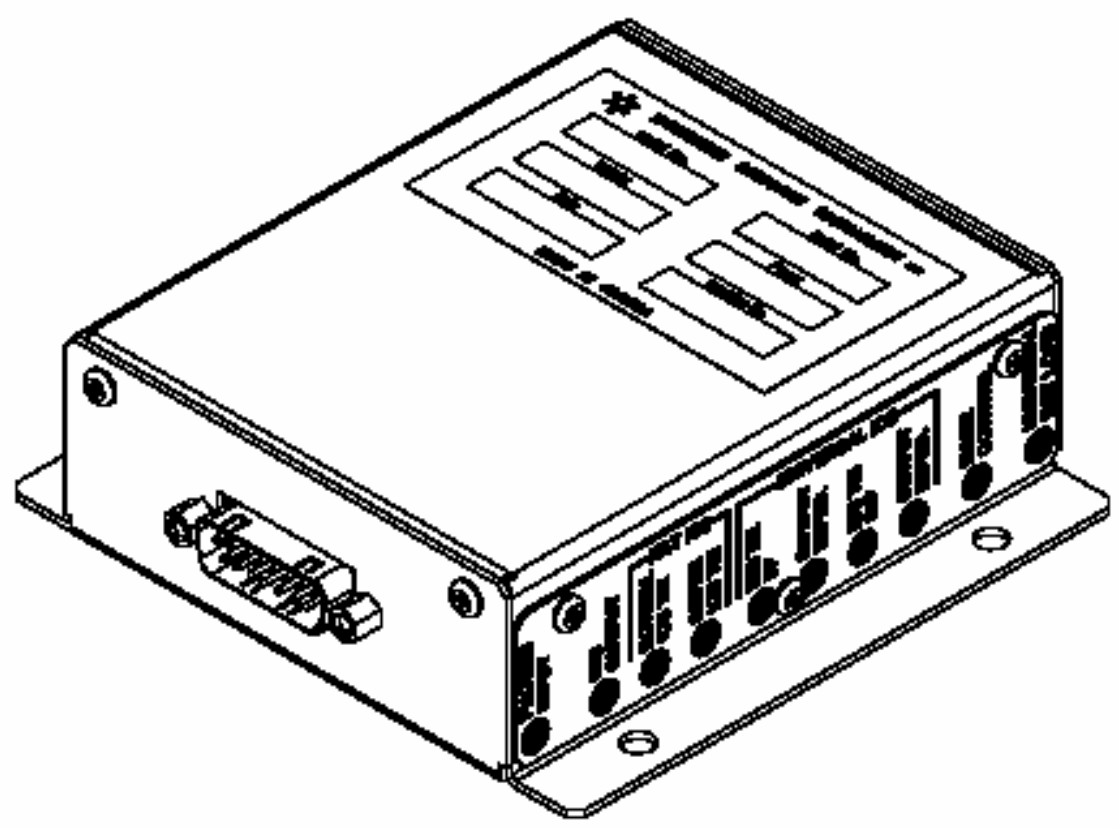

Figure 85 - Drawing of AA36-100 


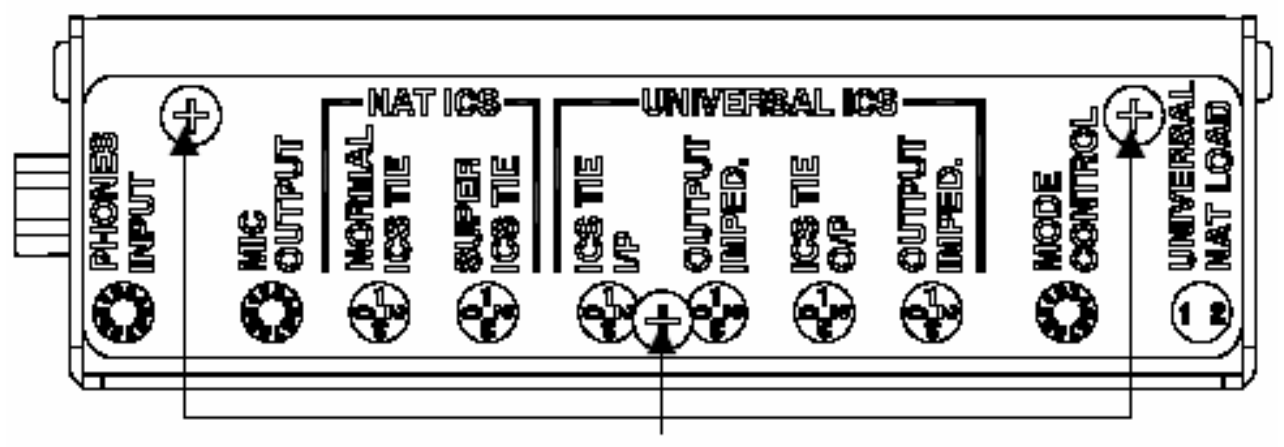

Cover Plate Screws

Figure 86 - NAT AA36-100 Side view

\section{B.10 NAT AA36-100 Settings}

The table below is the suggested settings for communication with the C-130 intercom system.

\begin{tabular}{|l|l|}
\hline Name & Setting \\
\hline Phones Input & 5.5 Vrms \\
\hline Mic Output & $0.25 \mathrm{Vrms}$ \\
\hline Normal ICS Tie & 3 NAT Loads \\
\hline Super ICS Tie & NAT Tie Line Position \\
\hline ICS Tie I/P & NAT Tie Line \\
\hline Output Impedance & NAT Tie Line \\
\hline ICS Tie O/P & NAT Tie Line \\
\hline Output Impedance & NAT Tie Line \\
\hline Mode Control & NAT Tie Line \\
\hline Universal NAT Load & NAT Tie Line \\
\hline
\end{tabular}

Table 7 - AA36-100 Settings 


\section{B.11 Flightcom Denali ANR \& Civilian Headset Specifications [18]}

\begin{tabular}{|c|c|c|c|}
\hline & & Denali Passive & Denali ANR \\
\hline \multirow{5}{*}{ 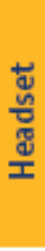 } & Shielding & $\begin{array}{l}\text { Full floating } \\
\text { w/independent ground }\end{array}$ & $\begin{array}{l}\text { Full floating } \\
\text { w/independent ground }\end{array}$ \\
\hline & Weight & 11.1 ounces & 13.4 ounces \\
\hline & Temperature Sensitivity & Not to exceed $156^{\circ} \mathrm{F}$ & Not to exceed $156^{\circ} \mathrm{F}$ \\
\hline & Battery Life & Not applicable & 20 hours of continuous use \\
\hline & Origin & Made in USA & Made in USA \\
\hline \multirow{6}{*}{ 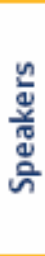 } & Sensitivity(@1mW in dBspl) & $104 \mathrm{~dB}$ & \\
\hline & Frequency Response & $90 \mathrm{~Hz}-20 \mathrm{kHz}$ & \\
\hline & Impedance & 300 ohms stereo/ch.- & mono \\
\hline & Total Harmonic Distortion (@1kHz) & $<.15 \%$ & \\
\hline & Maximum Power Input & $250 \mathrm{~mW}$ & \\
\hline & Noise Reduction Rating & $21 \mathrm{~dB}$ & \\
\hline \multirow{10}{*}{$\frac{\text { 응 }}{\frac{0}{\frac{0}{2}}}$} & Type & $\begin{array}{l}\text { Noise canceling electre } \\
\mathrm{w} / \text { constant-gain pream }\end{array}$ & \\
\hline & DC Bias Voltage & $8-16$ volts & \\
\hline & Supply Source Resistance & $220-2200$ ohms & \\
\hline & Frequency Response $( \pm 6 \mathrm{~dB})$ & $420 \mathrm{~Hz}-6.4 \mathrm{kHz}$ & \\
\hline & Sensitivity (@114dBspl*) & $1.3 \mathrm{~V}$ & \\
\hline & Noise Rejection Ratio (@1kHz) & $-46 \mathrm{~dB}$ & \\
\hline & Total Harmonic Distortion (@ 1kHz) & $<0.3 \%$ & \\
\hline & Impedance & 500 ohms & \\
\hline & Maximum Speech Level & $112 \mathrm{dBspl}^{*}$ & \\
\hline & Maximum Ambient Noise Level & $132 \mathrm{dBspl}^{*}$ & \\
\hline
\end{tabular}

* Sensitivity measurements referred to 0.0002 ubar (dynes $/ \mathrm{cm}^{2}$ ) @ $1 \mathrm{kHz}$. Microphone measurements made with 10 -volt supply with a $1000 \mathrm{ohm}$ resistor.

Contact Flightcom for the most current ANR specifications.

\section{B.12 Military Headset Specifications}

\begin{tabular}{|l|l|}
\hline Microphone & $\begin{array}{l}\text { Dynamic Mic(5 Ohm) M-87 } \\
\text { MIL2654212E }\end{array}$ \\
\hline Speaker & 150 Ohm Mono \\
\hline Connector & U-174/U 4-conductor plug \\
\hline
\end{tabular}




\section{B.13 Headset Plugs}

Listed below are the headset plugs and their pin outs. The Flightcom Denali were special ordered to contain a non-standard plug (see Figure 90) that is completely different than the military (Figure 89) and civilian (Figure 88) plugs. This special plug was necessary to distinguish between the military and civilian plugs and to have support for powering the active noise reduction circuitry.

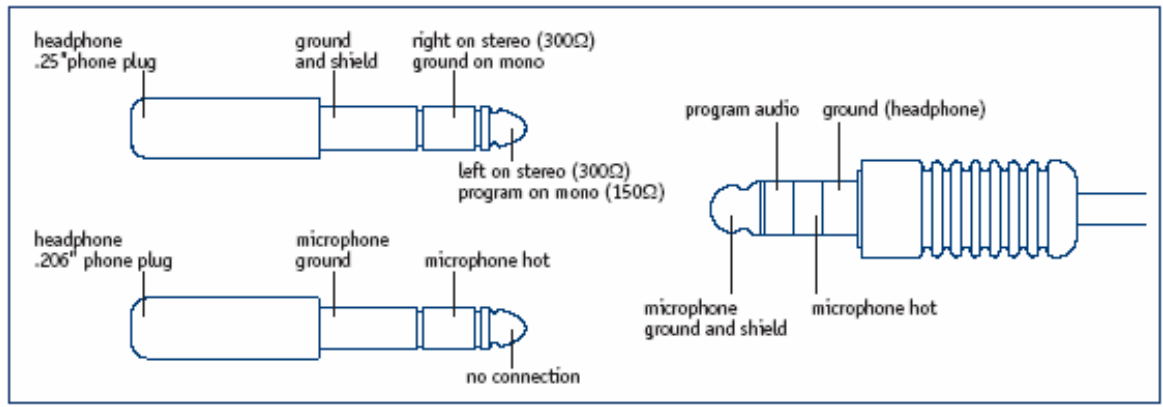

Figure 87 - Plug Pin outs

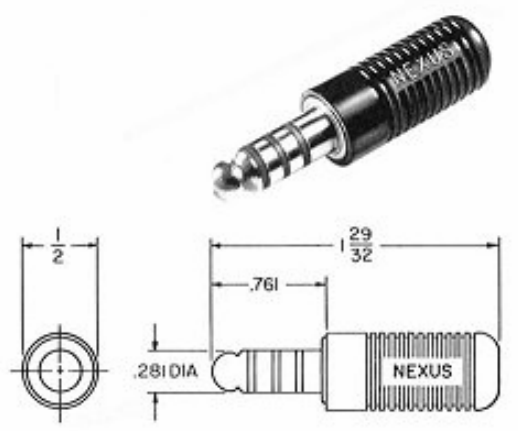

Figure 88 - Nexus TP-101 (U-174/U) Plug

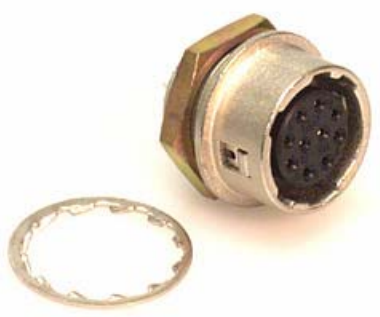

Figure 90 - Hirose HR10A-10R-10S[17]

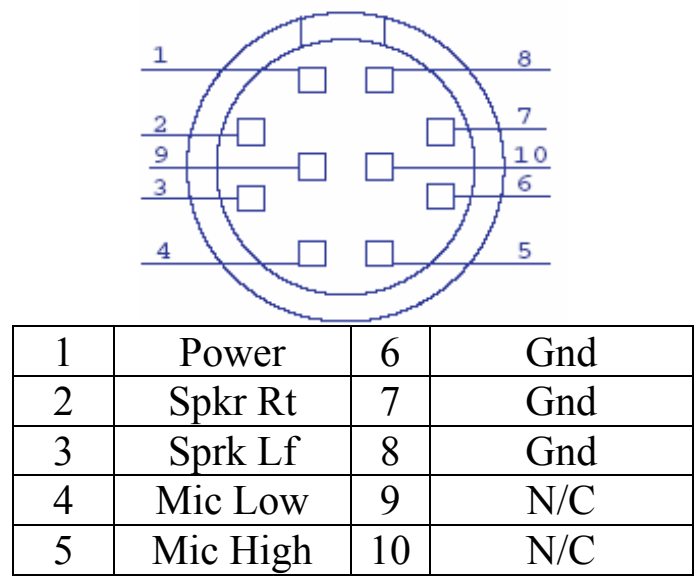

Figure 91 - Hirose HR10A-10R-10S Pinout 